\title{
Custodial symmetry violation in the SMEFT
}

\author{
Graham D. Kribs $\odot,{ }^{1}$ Xiaochuan Lu๑ ${ }^{1}$ Adam Martin, ${ }^{2}$ and Tom Tong $\odot^{1,3}$ \\ ${ }^{1}$ Institute for Fundamental Science and Department of Physics, University of Oregon, \\ Eugene, Oregon 97403, USA \\ ${ }^{2}$ Department of Physics, University of Notre Dame, South Bend, Indiana 46556 USA \\ ${ }^{3}$ Amherst Center for Fundamental Interactions, Department of Physics, University of Massachusetts \\ Amherst, Amherst, Massachusetts 01003, USA
}

(Received 2 April 2021; accepted 3 July 2021; published 7 September 2021)

\begin{abstract}
We investigate precision observables sensitive to custodial-symmetric/-violating UV physics beyond the Standard Model. We use the Standard Model effective field theory (SMEFT) framework which in general includes nonoblique corrections that require a generalization of the Peskin-Takeuchi $T$ parameter to unambiguously detect custodial symmetry/violation. We take a first step towards constructing a SMEFT reparametrization-invariant replacement, which we call $\mathscr{T}$, valid at least for tree-level custodial-violating contributions. We utilize a new custodial basis of $\nu$ SMEFT (SMEFT augmented by right-handed neutrinos) which explicitly identifies the global $S U(2)_{R}$ symmetries of the Higgs and fermion sectors, which in turn permits easy identification of higher-dimensional operators that are custodial preserving or violating. We carefully consider equation-of-motion redundancies that cause custodial-symmetric operators in one basis to be equivalent to a set of custodial-symmetric and/or -violating operators in another basis. Utilizing known results about tree/loop operator generation, we demonstrate that the basis-dependent appearance of custodial-violating operators does not invalidate our $\mathscr{T}$ parameter at tree level. We illustrate our results with several UV theory examples, demonstrating that $\mathscr{T}$ faithfully identifies custodial symmetry violation, while $T$ can fail.
\end{abstract}

DOI: 10.1103/PhysRevD.104.056006

\section{INTRODUCTION}

It is widely anticipated that there is new physics beyond the Standard Model (SM). In the absence of directly producing the new particles of the beyond-the-SM (BSM) sector, we would like to maximize the information we can glean about the UV physics from indirect probes. In this approach, the LEP era established the importance of electroweak precision data [1,2], which could test the SM to an accuracy of $\sim 0.1 \%$. Constraints on the scale of new physics can be $\Lambda \gtrsim 10 \mathrm{TeV}$ for those contributing to electroweak precision observables at the order $v^{2} / \Lambda^{2}$.

Directly calculating the contributions to electroweak precision observables from a given UV theory is in principle straightforward. However, it must be done on a case-by-case basis and consequently, does not (necessarily) provide general insights about the new physics. Peskin and Takeuchi demonstrated that the new physics effects can be efficiently categorized by utilizing three precision

Published by the American Physical Society under the terms of the Creative Commons Attribution 4.0 International license. Further distribution of this work must maintain attribution to the author(s) and the published article's title, journal citation, and DOI. Funded by SCOAP . parameters: $S, T$, and $U$ [3]. These parameters provide a simple, stunningly ubiquitous bridge between the effects of a new UV sector and electroweak precision data, and have become popular tests in determining the phenomenologically viable parameter space for BSM theories. In particular, the $T$ parameter is identified as the manifestation of "custodial" symmetry-breaking effects from the UV sector. Theories beyond the SM are often constructed to respect custodial symmetry in order to avoid the strong bounds on the $T$ parameter, including originally technicolor [4] (for a review, see Ref. [5]), as well as composite Higgs (e.g., Refs. [6-10]), little Higgs theories [11-15], and dark matter theories [16-18].

The Peskin-Takeuchi $T$ parameter can be constrained from a variety of electroweak data. There are two observables that are often associated with directly constraining $T$ : $\hat{\rho}_{*}(0)$, the ratio of charged current to neutral current $(\mathrm{CC} /$ NC) in the limit of zero momentum [3], and the Veltman $\hat{\rho} \equiv \frac{m_{W}^{2}}{m_{Z}^{2} \cos ^{2} \theta}$ [19]. We emphasize that these two are quite different observables ${ }^{1}$ despite often being confused with each other (see, e.g., Ref. [20]). In particular, $\hat{\rho}_{*}(0)$ only depends on $T$, where a nonzero value can be directly

\footnotetext{
${ }^{1}$ We thank S. Chang for emphasizing this point to us.
} 
associated with custodial violation. The Veltman $\hat{\rho}$, on the other hand, depends on all of $S, T$, and $U$, and can deviate from 1 due to custodial-symmetric UV effects (see Ref. [3]):

$$
\begin{gathered}
\hat{\rho}_{*}(0)-1=\alpha T \\
\hat{\rho}-1=\frac{\alpha}{\cos 2 \theta}\left(-\frac{1}{2} S+\cos ^{2} \theta T+\frac{\cos 2 \theta}{4 \sin ^{2} \theta} U\right) .
\end{gathered}
$$

In determining the strongest experimental constraints on $T$ (and $S, U$ ), the simplicity of Eq. (1a) may be outweighed by the precision on the Veltman $\hat{\rho}$ observable and associated $Z$ pole observables that can simultaneously constrain $S, T$, and $U$.

The $S, T, U$ parameters, however, have limitations. In particular, a key assumption, clearly stated at the time, is that the UV physics contributes only "obliquely," i.e., via the two-point functions of the SM electroweak gauge bosons. Another assumption is that the analysis only accounts for up to $p^{2}$ terms in gauge boson two-point functions. As precision increased, the framework was generalized to $p^{4}$ order by introducing the new parameters $V, W, X$, and $Y$ [21-24], though the oblique assumption remains in place.

Following the discovery of the Higgs boson [25,26], the Standard Model effective field theory (SMEFT) [27-29] has become a new popular framework for model-independent analyses of BSM physics, especially given the null results for the various direct BSM searches at the LHCIn this framework, new physics is considered as sufficiently heavy, such that it can be integrated out, resulting in higherdimensional operators, supplementing the SM Lagrangian. ${ }^{2}$

From the SMEFT point of view, only a very restricted set of UV theories (the so-called universal theories [24,42,43]) contribute only obliquely; fully general UV sectors captured by SMEFT also have nonoblique corrections [44]. In addition, even for universal theories, oblique corrections do not remain oblique: nonoblique corrections are generated as soon as renormalization group effects are included $[45,46]$. Therefore, a generalization of the $T$ parameter that does not rely on restricting to oblique-only corrections would be of significant importance to determine custodial symmetry or its violation of a generic UV theory.

\footnotetext{
${ }^{2}$ Classifying the general form of these operators has had a long history [28,29]. The "Warsaw" basis [30], for instance, provides a nonredundant parametrization of the set of all dim- 6 operators. Other operator bases, e.g., the SILH basis [10] can be related through integration-by-parts and equations-of-motion redundancies [31]. A systematic classification and counting of SMEFT operators has been recently achieved using the Hilbert series technique [32-36] up to dimension eight and beyond [37-40]. The number of operators grows rapidly with the dimension [39,40]. At dim-6, SMEFT contains 3045 operators [39,41], assuming all of the global symmetries of the SM are broken.
}

In this paper we present a first step in resolving this issue. Note that once we generalize beyond the oblique assumption, exactly what one means by custodial symmetry becomes more subtle and needs to be revisited. The $\mathrm{CC} / \mathrm{NC}$ ratio, universal for purely oblique corrections, now depends on what fermions are considered. Given this ambiguity in defining custodial symmetry, we make a choice that resembles the definition from $\hat{\rho}_{*}(0)$. Specifically, we define UV physics to be custodial symmetric when an $S U(2)_{R}$ global symmetry is preserved (in the limit of zero hypercharge coupling) by all UV interactions with the Higgs sector of the SM.

In the SMEFT framework, one works with effective operators whose constituents have manifest transformation properties under the global $S U(2)_{R}$ symmetries in the SM Higgs and/or fermion sectors. In this sense, the Wilson coefficients are superior to the $S, T, U$ parameters, as they can directly indicate $S U(2)_{R}$ symmetries or their violation. This is a simplification compared to the oblique framework, where one has to infer the $S U(2)_{R}$ symmetry from the $\mathrm{CC} /$ $\mathrm{NC}$ ratio. To better utilize this feature of SMEFT, we take linear combinations of operators in the dimension-six (dim6) Warsaw basis, extended to include right-handed neutrinos (thus $\nu$ SMEFT, rather than SMEFT), and map them into a new custodial basis where all operators have manifest transformation properties under the global $S U(2)_{R}$ symmetries in the SM. This allows us to directly identify UV custodial symmetry/violation from the Wilson coefficients generated by matching.

Assisted by the custodial $(\nu)$ SMEFT basis, we construct a linear combination of Wilson coefficients that we call $\mathscr{T}_{l}$, a new precision parameter that serves as a generalization of the $T$ parameter to indicate custodial symmetry/violation in nonoblique theories. We show that $\mathscr{T}_{l}$ can be constructed from $Z$-pole observables and $m_{W}$, faithfully determining at tree level if the UV sector contains "hard" custodial violation (that persists even when the hypercharge gauge coupling vanishes), independent of whether the UV sector contributes only obliquely. Importantly, as our new $\mathscr{T}_{l}$ parameter involves multiple electroweak observables. As a consequence, the constraint on custodial violation that it sets is only as strong as the weakest link, namely, that the least precise component observable determines the true bound on custodial violation of UV physics.

Our new parameter $\mathscr{T}_{l}$ is a first step only, as it does not capture loop corrections from the SM or SMEFTİn particular, modifications to the parameter are required to account for the known SM violation of custodial symmetry at the one-loop level (arising mainly from top-loop contributions). Furthermore, incorporating loop-level effects in SMEFT requires a substantial amount of additional effort due to an ambiguity that arises from equation-of-motion (EOM) redundancies. When custodial-symmetric UV physics is integrated out, it generates custodial-symmetric operators, but not necessarily in the Warsaw or custodial 
basis of $(\nu)$ SMEFT. Ordinarily one simply utilizes integration-by-parts (IBP) and EOM redundancies to rewrite the UV-generated operators in terms of whatever basis one prefers, in our case our custodial basis of $(\nu)$ SMEFT. However, the EOM redundancy can trade custodial-symmetric operators for custodial-violating operators proportional to the SM violation of custodial symmetry. This is simply because the EFT as a whole does not respect custodial symmetry, even if the integrated-out UV physics does. This could have sunk any chance to isolate observables only sensitive to $U V$ sector violations of custodial symmetry. Fortunately, from known results about tree/loop operator generation [47-49], we find that restricting to treelevel-generated operators, our set of observables, and hence our $\mathscr{T}_{l}$ parameter, remain faithful in identifying hard custodial violation of UV physics. This is the main result of this paper.

The layout of the rest of this paper is as follows. In Sec. II we establish notation and review the global $S U(2)_{R}$ symmetries of the Higgs and fermion sectors of the SM, as well as how they are broken by various interactions. This will help us lay out our working definition of custodial symmetry. Next, in Sec. III we introduce the custodial basis for $(\nu)$ SMEFT and classify operators in that basis according to their properties under our custodial symmetry. We also provide mapping between this basis and conventional SMEFT bases which will be useful for quickly importing past results. In Sec. IV we select a set of electroweak precision observables and determine a particular combination of them that is sensitive to hard UV custodial violation at tree level. Our new electroweak precision parameter $\mathscr{T}_{l}$ arises from this combination, and serves as a generalization of the $T$ parameter to $\mathrm{UV}$ theories with nonoblique corrections. This section contains our main results. The impact (or lack thereof) of EOM redundancies is the subject of Sec. V. In Sec. VI we investigate several example UV theories to demonstrate the validity of our new $\mathscr{T}_{l}$ parameter constructed in Sec. IV. Finally, we conclude in Sec. VII.

\section{II. $S U(2)_{R}$ SYMMETRIES IN $(\nu)$ SM AND CUSTODIAL SYMMETRY}

In this section, we discuss the (approximate) global $S U(2)_{R}$ symmetries in $(\nu) \mathrm{SM}$ (Secs. II A and II B), identify their breaking sources (Sec. II C), and then introduce our definition of the custodial symmetry (Sec. II D).

Let us first establish our notation for the group theory and field content. We use $\tau^{a}=\tau_{R}^{a}=\sigma^{a}$ with $a=1,2,3$ to denote Pauli matrices. The $S U(2)_{L}$ and $S U(2)_{R}$ generators in the fundamental representation are hence $t^{a}=\frac{1}{2} \tau^{a}$ and $t_{R}^{a}=\frac{1}{2} \tau_{R}^{a}$, respectively. The $S U(3)_{c}$ generators in the fundamental representation are denoted by $T^{A}$ with $A=1, \ldots, 8$. The SM covariant derivative is

$$
D_{\mu}=\partial_{\mu}-i g_{3} G_{\mu}^{A} T^{A}-i g_{2} W_{\mu}^{a} t^{a}-i g_{1} B_{\mu} \mathrm{y},
$$

with y denoting the hypercharge, $G_{\mu}^{A}, W_{\mu}^{a}, B_{\mu}$ denoting the gauge fields, and $g_{3}, g_{2}, g_{1}$ denoting the gauge couplings. A general field strength is denoted as $X_{\mu \nu} \in\left\{G_{\mu \nu}^{A}, W_{\mu \nu}^{a}, B_{\mu \nu}\right\}$. For the dual, we adopt the convention $\tilde{X}_{\mu \nu} \equiv \frac{1}{2} \epsilon_{\mu \nu \alpha \beta} X^{\alpha \beta}$, with $\epsilon_{0123}=+1$. We use the usual Dirac matrices $\gamma^{\mu}$, and $\sigma^{\mu \nu} \equiv \frac{i}{2}\left[\gamma^{\mu}, \gamma^{\nu}\right]$.

Our notation for the SM Lagrangian is

$$
\begin{aligned}
\mathcal{L}_{\mathrm{SM}}= & -\frac{1}{4} G_{\mu \nu}^{A} G^{A \mu \nu}-\frac{1}{4} W_{\mu \nu}^{a} W^{a \mu \nu}-\frac{1}{4} B_{\mu \nu} B^{\mu \nu}+|D H|^{2}-\lambda\left(|H|^{2}-\frac{1}{2} v^{2}\right)^{2} \\
& +\sum_{\psi} \bar{\psi} i \not \emptyset \psi-\left(Y_{u} \bar{q} \tilde{H} u+Y_{d} \bar{q} H d+Y_{e} \bar{l} H e+\text { H.c. }\right),
\end{aligned}
$$

where for the SM fermions $\psi$ we follow Ref. [30] to use $\{q, l\}$ for left-handed $S U(2)_{L}$ doublets and $\{u, d, e\}$ for right-handed $S U(2)_{L}$ singlets. In the above, the Yukawa couplings $Y_{u}, Y_{d}, Y_{e}$ are $3 \times 3$ matrices in the generation space, but we have suppressed the generation indices for compactness. We can also extend the SM to include righthanded neutrinos $\nu$ - what we refer to as $\nu \mathrm{SM}$. In this case, the Lagrangian is augmented as

$$
\mathcal{L}_{\nu \mathrm{SM}}=\mathcal{L}_{\mathrm{SM}}+\bar{\nu} i \not D \nu-\left(Y_{\nu} \bar{l} \tilde{H} \nu+\text { H.c. }\right) .
$$

A Majorana mass for the right-handed neutrinos can be written, $M \bar{\nu} \nu$, which is allowed by the gauge symmetries, but violates lepton number by 2 units. For this term to be present in the low-energy effective theory, the Majorana mass scale $M$ must be less than the cutoff scale of ${ }$ SMEFT. $^{3}$

\section{A. Higgs sector: $S U(2)_{R H}$}

We begin our discussion of global $S U(2)_{R}$ symmetries with the Higgs doublet

\footnotetext{
${ }^{3}$ As we will see, none of the observables that we consider depend on the presence or absence of a Majorana mass smaller than the cutoff scale of the effective theory.
} 


$$
H=\left(\begin{array}{c}
G^{+} \\
\left(v+h+i G^{0}\right) / \sqrt{2}
\end{array}\right) .
$$

The Higgs potential is invariant under an $S O(4)$ symmetry,

$$
S O(4) \sim S U(2)_{L} \times S U(2)_{R H},
$$

where $S U(2)_{L}$ and the $t_{R}^{3}$ generator of $S U(2)_{R H}$ are gauged in $(\nu)$ SM. This symmetry is spontaneously broken to $S O(3) \sim S U(2)_{V}$ when the Higgs develops a vacuum expectation value (VEV).

We can reexpress the Higgs field in terms of a $(2,2)$ bifundamental scalar field that transforms under the $\left(U_{L}, U_{R}\right) \in S U(2)_{L} \times S U(2)_{R H}$ as $^{4}$

$$
\begin{aligned}
\Sigma & \equiv\left(\begin{array}{cc}
\tilde{H} H & H
\end{array}\right) \\
& =\left(\begin{array}{cc}
\left(v+h-i G^{0}\right) / \sqrt{2} & G^{+} \\
-G^{-} & \left(v+h+i G^{0}\right) / \sqrt{2}
\end{array}\right) \rightarrow U_{L} \Sigma U_{R}^{\dagger} .
\end{aligned}
$$

In principle, all interactions are built out of $\Sigma$, but it is sometimes helpful to make use of the identity

$$
\Sigma_{i_{R} i_{L}}^{\dagger} \equiv \epsilon_{i_{R} j_{R}} \epsilon_{i_{L} j_{L}} \Sigma_{j_{L} j_{R}}
$$

and write operators with $\Sigma^{\dagger}$, where the $S U(2)$ transformation properties are easier to recognize. For example, the SM Higgs potential can be written as

$$
V=\lambda\left(|H|^{2}-\frac{v^{2}}{2}\right)^{2}=\frac{\lambda}{4}\left[\operatorname{tr}\left(\Sigma^{\dagger} \Sigma\right)-v^{2}\right]^{2},
$$

where the $S U(2)_{L} \times S U(2)_{R H}$ symmetry is manifest. Similarly, one can rewrite the Higgs $S U(2)_{L}$ and $U(1)_{Y}$ currents as

$$
\begin{gathered}
H^{\dagger} i \stackrel{\leftrightarrow}{D}{ }_{\mu}^{a} H \equiv H^{\dagger} \tau^{a} i D_{\mu} H+\text { H.c. }=\operatorname{tr}\left(\Sigma^{\dagger} \tau^{a} i D_{\mu} \Sigma\right), \\
H^{\dagger} i \stackrel{\leftrightarrow}{D_{\mu}} H \equiv H^{\dagger} i D_{\mu} H+\text { H.c. }=-\operatorname{tr}\left(\Sigma^{\dagger} i D_{\mu} \Sigma \tau_{R}^{3}\right),
\end{gathered}
$$

where the $S U(2)$-preserving/-violating structures are more explicit.

\section{B. Fermion sector: $S U(2)_{R q_{R}}, S U(2)_{R l_{R}}$}

Turning to the fermion sector of the SM, there are several approximate $S U(2)_{R}$ symmetries that become exact in the limit of neglecting hypercharge coupling $g_{1}$ and the Yukawa couplings. Focusing on one generation of fermions

\footnotetext{
${ }^{4}$ Here $\tilde{H} \equiv i \sigma^{2} H^{*}=\epsilon H^{*}$, which transforms in the same way as $H$ itself under the $S U(2)_{L}$ symmetry; $\epsilon_{i j}=-\epsilon_{j i}$ is an $S U(2)$ invariant tensor, and we take $\epsilon_{12}=+1$.
}

for the moment, the right-handed up-type quark $u$ and downtype quark $d$ can be grouped together to form a doublet

$$
q_{R} \equiv\left(\begin{array}{l}
u \\
d
\end{array}\right)
$$

which has a $U(2)_{q_{R}}$ global symmetry. We can break this symmetry into the baryon number $U(1)_{B}$ and a global $S U(2)_{R}$ quark isospin symmetry that we will call $S U(2)_{R q_{R}}$ :

$$
U(2)_{q_{R}}=U(1)_{B} \times S U(2)_{R q_{R}} .
$$

Similarly, when the SM is extended to $\nu \mathrm{SM}$, we can build a right-handed lepton doublet

$$
l_{R} \equiv\left(\begin{array}{l}
\nu \\
e
\end{array}\right)
$$

which has a global $U(2)_{l_{R}}$ symmetry that we identify as consisting of lepton number and isospin:

$$
U(2)_{l_{R}}=U(1)_{L} \times S U(2)_{R l_{R}}
$$

In the case of three generations, we will get the quark isospin $S U(2)_{R q_{R}}$ and the lepton isospin $S U(2)_{R l_{R}}$ for each generation.

\section{C. $S U(2)_{R}$ violation in $(\nu) S M$}

With the global $S U(2)_{R}$ symmetries in $(\nu)$ SM identified, we can now classify the symmetry-breaking sources, namely, Yukawa couplings and the gauging of hypercharge. For simplicity, we will focus on the one-generation case.

Yukawa couplings play two roles: 1) they tie the Higgs $S U(2)_{R H}$ symmetry to the isospin symmetries $S U(2)_{R q_{R}}$, $S U(2)_{R l_{R}}$, and 2) they break these symmetries. To disentangle these two effects, we can first write the Yukawa interactions in terms of the bidoublet Higgs $\Sigma$, e.g., for quarks,

$$
Y_{u} \bar{q} \tilde{H} u+Y_{d} \bar{q} H d=\bar{q} \Sigma\left(\begin{array}{cc}
Y_{u} & 0 \\
0 & Y_{d}
\end{array}\right) q_{R},
$$

and then split the above Yukawa matrix as

$$
\left(\begin{array}{cc}
Y_{u} & 0 \\
0 & Y_{d}
\end{array}\right)=\frac{Y_{u}+Y_{d}}{2} \mathbb{1}_{2 \times 2}+\frac{Y_{u}-Y_{d}}{2} \tau_{R}^{3}
$$

This way, the symmetry-breaking pattern becomes clear. The term proportional to $\mathbb{1}_{2 \times 2}$ leads to $S U(2)_{R H} \times$ $S U(2)_{R q_{R}} \rightarrow S U(2)$, while the $\tau_{R}^{3}$ term breaks $S U(2)_{R H} \times$ $S U(2)_{R q_{R}} \rightarrow U(1)$. By the same logic, the Yukawa interactions in the lepton sector of $\nu \mathrm{SM}$ can be grouped into a combination that ties $S U(2)_{R H}$ to $S U(2)_{R l_{R}}$ and a

\footnotetext{
${ }^{5}$ Similar arguments hold for the case of three generations.
} 
combination that breaks $S U(2)_{R H} \times S U(2)_{R l_{R}} \rightarrow U(1)$. [A Majorana mass for the right-handed neutrinos explicitly breaks $S U(2)_{R l_{R}}$ to nothing.] The matrices $\mathbb{1}_{2 \times 2}$ and $\tau_{R}^{3}$ will appear often in this work, so we will adopt the convenient shorthand

$$
P_{+} \equiv \mathbb{1}_{2 \times 2}, \quad P_{-} \equiv \tau_{R}^{3} .
$$

We will use $P_{ \pm}$to apply to both $S U(2)_{R q_{R}}$ and $S U(2)_{R l_{R}}$ spaces; exactly which space we are working with should be clear from the context.

On the other hand, gauging hypercharge corresponds to gauging the $\tau_{R}^{3}$ generators of $S U(2)_{R H}, S U(2)_{R q_{R}}$, and $S U(2)_{R l_{R}}$. This breaks all of them simultaneously down to $U(1)_{Y}$ - exactly the $U(1)$ left intact by $P_{-}$from Yukawa breaking.

\section{Custodial $S U(2)_{R}$}

Now that we have identified the $S U(2)_{R}$ symmetries and violation in $(\nu) \mathrm{SM}$, we are ready to precisely define custodial symmetry in this paper:

\section{UV physics is custodial symmetric when there is a}

global $S U(2)_{R}$ symmetry preserved, in the limit $g_{1} \rightarrow 0$,

by all UVinteractions with the Higgs sector of the SM.

Here, the preserved $S U(2)_{R}$ symmetry could be either $S U(2)_{R H}$ itself or a diagonal subgroup of $S U(2)_{R H} \times$ $S U(2)_{R q_{R}}, S U(2)_{R H} \times S U(2)_{R l_{R}}$, or $S U(2)_{R H} \times S U(2)_{R q_{R}} \times$ $S U(2)_{R l_{R}}$. That is, the $S U(2)_{R}$ group must involve $S U(2)_{R H}$ in some way. A few important comments are in order about this definition:

(1) Our definition is exclusively about the UV sector. Therefore, even in the case where the UV sector respects custodial symmetry, the identified $S U(2)_{R}$ symmetry is still not an exact symmetry of the whole Lagrangian [the UV sector plus $(\nu)$ SM. In particular, the hypercharge coupling $g_{1}$ and the mismatch in Yukawa couplings $\left[Y_{u}-Y_{d}\right.$ and $Y_{\nu}-Y_{e}$; see, e.g., Eq. (16)] in $\nu$ SM break it. Only in the limits $g_{1} \rightarrow 0$, $Y_{u}-Y_{d} \rightarrow 0, \quad Y_{\nu}-Y_{e} \rightarrow 0$, will the custodial $S U(2)_{R}$ become an exact symmetry of the entire $\mathrm{UV}+\mathrm{SM}$ theory.

(2) Also, because our definition is exclusive to the UV interactions, whether or not a UV sector is adjudicated to be custodial symmetric does not depend on the presence or absence of $(\nu)$ SM Yukawa couplings. By contrast, the hypercharge coupling $g_{1}$ could play a role, as it could participate in the UV interactions when some UV particles have nonzero hypercharge.

(3) The breaking of custodial $S U(2)_{R}$ by the UV interactions can thus be categorized as follows:

(a) "Soft" breakings that vanish in the limit $g_{1} \rightarrow 0$.

(b) "Hard" breakings that persist in the limit $g_{1} \rightarrow 0$.
In our definition above, a UV sector with "soft" custodial $S U(2)_{R}$ breaking is defined as custodial symmetric. This is because our interest in this paper is "hard" custodial violation. In the rest of this paper, we will utilize this terminology strictly unless explicitly stated otherwise, namely, that our "custodial-violating UV physics" contains "hard" custodial breakings, and our "custodial-symmetric UV physics" allows for soft breakings.

In the above, we have established a definition of the custodial symmetry for $U V$ physics. However, as explained in the Introduction, we are not interested in analyzing UV theories case by case. Instead, we would like to follow the spirit of the electroweak precision parameters $S, T, U$, and use a general framework to analyze UV physics independent of the UV model. In this paper, the framework we use is dim-6 $(\nu)$ SMEFT. This motivates us to divide the dim-6 $(\nu)$ SMEFT operators into two categories: "custodialpreserving/-violating operators" that can/cannot be possibly generated by custodial-symmetric UV physics.

Usually, a symmetry possessed by the UV theory gets inherited by the EFT (as long as the heavy states integrated out do not break it). In the case of our custodial $S U(2)_{R}$, however, the situation is less straightforward, precisely because it is not an exact symmetry of the whole UV Lagrangian. Nevertheless, if we restrict ourselves to the leading matching order (which could be tree level, one-loop level, or even higher, depending on the UV theory), there are only heavy particle propagators in the contributing diagrams, and hence only UV interactions beyond $(\nu) \mathrm{SM}$ will participate. In this case, all of the resulting $(\nu)$ SMEFT operators will preserve the custodial $S U(2)_{R}$ symmetry. For the rest of the paper, we only consider the leading matching order unless explicitly stated otherwise. This allows us to make the above desired $(\nu)$ SMEFT operator division simply based on their $S U(2)_{R}$ transformation properties, a task we will tackle in the next section.

\section{CUSTODIAL BASIS OF ( $\nu)$ SMEFT}

In this section, we introduce a new basis for dim- 6 ( $\nu$ )SMEFT - the custodial basis - to facilitate the identification of operators that preserve/violate the custodial symmetry. Using this basis, we then identify the operators that can/cannot be possibly generated by integrating out custodial-symmetric UV sectors.

Our presentation of the operator basis largely follows Refs. [30,41,50,51], extended to include right-handed neutrinos [52]. As preparation, we first present in Table II all of the independent baryon-number-preserving and lepton-number-preserving operators in the Warsaw basis for $\nu$ SMEFT (suppressing flavor indices). ${ }^{6}$ In addition to the $76=42+(17+$ H.c. $)$ SMEFT operators, there are

\footnotetext{
${ }^{6}$ For easy reading/contrasting, we have gathered all of the tables of operator bases and the relevant translation dictionaries in Appendix C.
} 
$25=7+(9+$ H.c. $)$ new operators involving right-handed neutrinos. Reducing $\nu$ SMEFT back to SMEFT is straightforward by restricting appropriate Wilson coefficients to zero, which we show in Table VII.

Now we build the custodial basis. Our basic approach is to recombine the Warsaw basis operators $Q_{i}$ such that their transformation properties under the global $S U(2)_{R H}$ and isospin symmetries $S U(2)_{R q_{R}}, S U(2)_{R l_{R}}$ become manifest, close to what we did for $\nu \mathrm{SM}$ in Eqs. (15) and (16). It is also worth mentioning that a similar rewriting of dim-6 SMEFT in other bases exists; see, e.g., [53]. Performing this recombination for all of the operators in Table II, we arrive at our custodial basis operators $O_{i}$, summarized in Table III. An explicit translation dictionary between the two operator bases is further given in Table IV. Many operators do not change in going from the Warsaw basis to the custodial basis. In particular, operators built purely out of $S U(2)_{R}$ singlets translate trivially. All operators that involve exclusively the left-handed fermion fields of the SM fall into this category. On the other hand, significant differences from the Warsaw basis can be observed in the operators involving the right-handed fermion fields.

From the translation dictionary in Table IV, we can also easily determine the corresponding relations between the Wilson coefficients in these two bases:

$$
\mathcal{L}_{\mathrm{EFT}}-\mathcal{L}_{\mathrm{SM}}=\sum_{i} a_{i} O_{i}=\sum_{i} C_{i} Q_{i}
$$

We provide explicit translation dictionaries between the Wilson coefficients in Tables V and VI. Note that we have absorbed the scale suppressing the $Q_{i}$ and $O_{i}$ into the Wilson coefficients, making them dimensionful, $\left[C_{i}\right]=\left[a_{i}\right]=-2$. This is a bit unconventional, but it compactifies the notation. One can express our results in terms of dimensionless Wilson coefficients and a new physics scale $\Lambda$ by replacing $C_{i} \rightarrow$ $\tilde{C}_{i} / \Lambda^{2}, a_{i} \rightarrow \tilde{a}_{i} / \Lambda^{2}$ everywhere.

We now wish to identify operators in Table III that can/ cannot be possibly generated by custodial-symmetric UV physics. Recall that in the limit $g_{1} \rightarrow 0$, custodial-symmetric UV physics preserves an $S U(2)_{R}$ symmetry. Consequently, in this limit, only operators that preserve the same $S U(2)_{R}$ symmetry could be generated by matching (at the leading order). However, there are four possibilities for this $S U(2)_{R}$ :

(1) $S U(2)_{R H}$.

(2) The diagonal subgroup of $S U(2)_{R H} \times S U(2)_{R q_{R}}$.

(3) The diagonal subgroup of $S U(2)_{R H} \times S U(2)_{R l_{R}}$.

(4) The diagonal subgroup of $S U(2)_{R H} \times S U(2)_{R q_{R}} \times$ $S U(2)_{R l_{R}}$.

Therefore, if an operator in Table III preserves any of the four $S U(2)_{R}$ 's above in the limit $g_{1} \rightarrow 0$, then it can potentially be generated, and should be categorized as a "custodial-preserving operator." For example, in the limit $g_{1} \rightarrow 0$, the operator

$$
O_{H l_{R}}^{(3)+} \equiv \operatorname{tr}\left(\Sigma^{\dagger} i D_{\mu} \Sigma \tau_{R}^{a}\right)\left(\bar{l}_{R} \gamma^{\mu} \tau_{R}^{a} P_{+} l_{R}\right)
$$

preserves the diagonal subgroup of $S U(2)_{R H} \times S U(2)_{R l_{R}}$, and hence is a custodial-preserving operator. Note in particular that any operator with an explicit $B_{\mu \nu}$ should be understood as accompanied by a power of $g_{1}$. Therefore, in the limit $g_{1} \rightarrow 0$ these operators vanish, and so they are classified as custodial-preserving operators as well.

\section{A. Flavor indices of the Wilson coefficients}

In Tables II-VII we have suppressed all of the flavor indices, but it should be understood that each fermion field actually comes with a generation index, so are the corresponding Wilson coefficients. For example, the twofermion operator $Q_{H l}^{(3)}$ and four-fermion operator $Q_{l l}$ should actually read

$$
\begin{gathered}
Q_{p r}^{(3)}=\left(H^{\dagger} i \stackrel{\leftrightarrow a}{D_{\mu}^{a}} H\right)\left(\bar{l}_{p} \gamma^{\mu} \tau^{a} l_{r}\right), \\
Q_{p r s t}=\left(\bar{l}_{p} \gamma_{\mu} l_{r}\right)\left(\bar{l}_{s} \gamma^{\mu} l_{t}\right) .
\end{gathered}
$$

The EFT Lagrangian therefore has a sum over these generation indices:

$$
\begin{aligned}
& \mathcal{L}_{\mathrm{EFT}} \supset \sum_{p, r=1}^{3} C_{p l}^{(3)} Q_{p r}^{(3)}+\sum_{p, r, s, t=1}^{3} C_{p r s t}{ }_{p r s} Q_{p r s t} \\
& =\sum_{p, r=1}^{3} a_{p l}^{(3)} O_{p l}^{(3)}+\sum_{p r}^{3} a_{p, r, s, t=1}{ }_{p r s t} O_{p r s t}^{u} .
\end{aligned}
$$

However, we often suppress the flavor indices when it is clear from the context.

As we will see, most four-fermion operators do not contribute to the observables to be discussed in Sec. IV. However, one exception is the mixed first- and secondgeneration four-lepton operator, which contributes to $\hat{G}_{F}$. We give this operator a special name for future convenience:

$$
Q_{12} \equiv\left(\bar{l}_{1} \gamma_{\mu} l_{2}\right)\left(\bar{l}_{2} \gamma^{\mu} l_{1}\right) \equiv O_{12}
$$

Clearly, the corresponding Wilson coefficients are related to our general notation as

$$
C_{12}=C_{1221}^{u}+C_{2112}^{u}=a_{1221}^{u}+a_{2112}^{u}=a_{12}
$$

\section{OBSERVABLES SENSITIVE TO CUSTODIAL SYMMETRY/VIOLATION IN $(\nu)$ SMEFT}

In this section, we study an example set of precision observables that will allow us to identify whether the UV physics contain hard custodial violation: 


$$
\left\{\hat{\alpha}, \hat{G}_{F}, \hat{m}_{Z}^{2}, \hat{m}_{W}^{2}, \hat{\Gamma}_{Z L_{L} \bar{\nu}_{L}}, \hat{\Gamma}_{Z e_{L} \bar{e}_{L}}, \hat{\Gamma}_{Z e \bar{e}}\right\}
$$

In order, these are the (electromagnetic) fine-structure constant, the Fermi constant, the pole masses of the $Z$ and $W$ bosons, and the partial decay widths of the $Z$ boson to left-handed neutrinos, left-handed electrons, and righthanded electrons.

In what follows, we compute corrections from dim-6 ( $\nu$ SMEFT operators to these observables in Secs. IV A and IV B, at leading matching order (tree level in SMEFT), and then in Sec. IV C we construct from them a $T$ parameter generalization $\mathscr{T}_{l}$ that serves as an indicator of custodial violation in general $(\nu)$ SMEFT. Reparametrization invariance (RPI) plays an important role in our construction of $\mathscr{T}_{l}$, which we will explain in Sec. IV D. Our "observables" here refer to quantities that we can calculate in the $(\nu) \mathrm{SM} /(\nu) \mathrm{SMEFT}$ that do not depend on the choice of operator basis, and they can in principle be measured by experiments. Some of these observables can be directly measured, such as $\hat{\alpha}, \hat{G}_{F}, \hat{m}_{Z}^{2}, \hat{m}_{W}^{2}$, while others need to be inferred from other measurements. In Sec. IV $\mathrm{E}$ we discuss how to extract these observables from experimental measurements. The observable set chosen in Eq. (24) is only a demonstration example. There are more observables available in the canonical LEP choice, such as the hadronic branching ratio, the bottom quark branching ratio, or the total decay width of $Z$. We discuss these observables in Appendix B.

\section{A. Observables in the SM}

In the SM, the observables in Eq. (24) are given by the three Lagrangian parameters $g_{1}, g_{2}, v$ as ${ }^{7}$

$$
\begin{gathered}
\hat{\alpha}_{\mathrm{SM}}=\frac{g_{1}^{2} g_{2}^{2}}{4 \pi\left(g_{1}^{2}+g_{2}^{2}\right)}, \\
\hat{G}_{F, \mathrm{SM}}=\frac{1}{\sqrt{2} v^{2}}, \\
\hat{m}_{Z, \mathrm{SM}}^{2}=\frac{1}{4}\left(g_{1}^{2}+g_{2}^{2}\right) v^{2}, \\
\hat{m}_{W, \mathrm{SM}}^{2}=\frac{1}{4} g_{2}^{2} v^{2}, \\
\hat{\Gamma}_{Z \nu_{L} \bar{\nu}_{L}, \mathrm{SM}}=\frac{\hat{m}_{Z, \mathrm{SM}}}{96 \pi} \frac{g_{2}^{2}}{c_{\theta}^{2}}, \\
\hat{\Gamma}_{Z e_{L} \bar{e}_{L}, \mathrm{SM}}=\frac{\hat{m}_{Z, \mathrm{SM}}}{96 \pi} \frac{g_{2}^{2}}{c_{\theta}^{2}} c_{2 \theta}^{2},
\end{gathered}
$$

\footnotetext{
${ }^{7}$ Throughout this paper, we neglect the lepton masses in $Z$ decay widths.
}

$$
\hat{\Gamma}_{Z e \bar{e}, \mathrm{SM}}=\frac{\hat{m}_{Z, \mathrm{SM}}}{24 \pi} \frac{g_{2}^{2}}{c_{\theta}^{2}} s_{\theta}^{4}
$$

where $\theta$ denotes the Weinberg angle

$c_{\theta}=\cos \theta \equiv \frac{g_{2}}{\sqrt{g_{1}^{2}+g_{2}^{2}}}, \quad s_{\theta}=\sin \theta \equiv \frac{g_{1}}{\sqrt{g_{1}^{2}+g_{2}^{2}}}$.

\section{B. Observables in SMEFT}

Since the SM has only three inputs, the full set in Eqs. (25a)- $(25 \mathrm{~g})$ can be completely determined in terms of any subset of three observables. Typically, the most precisely measured subset is chosen: $\left\{\hat{\alpha}, \hat{m}_{Z}^{2}, \hat{G}_{F}\right\}$ or $\left\{\hat{m}_{W}^{2}, \hat{m}_{Z}^{2}, \hat{G}_{F}\right\}$. Once we include the contributions from SMEFT operators, three observables are no longer enough, as all of Eqs. (25a)-(25g) will be polluted with different combinations of Wilson coefficients $C_{i}$. Said another way, it is still possible to swap out $g_{1}, g_{2}$, and $v$ for $\left\{\hat{\alpha}, \hat{m}_{Z}^{2}, \hat{G}_{F}\right\}$ or $\left\{\hat{m}_{W}^{2}, \hat{m}_{Z}^{2}, \hat{G}_{F}\right\}$; however, in the presence of SMEFT effects, $g_{1}, g_{2}$, and $v$ will be functions of $C_{i}$ rather than numbers fixed by experiment. This $C_{i}$ dependence is referred to as the "electroweak input shifts" in the literature. The exact form of the shifts depends on which three observables are used to solve for $g_{1}, g_{2}$, and $v$ : either the $\hat{\alpha}$ scheme $\left(\left\{\hat{\alpha}, \hat{m}_{Z}^{2}, \hat{G}_{F}\right\}\right)$ or the $\hat{m}_{W}$ scheme $\left(\left\{\hat{m}_{W}^{2}, \hat{m}_{Z}^{2}, \hat{G}_{F}\right\}\right){ }^{8}$ In this paper, we will exclusively use the $\hat{\alpha}$ scheme. Of course, the input shifts are only one place Wilson coefficients can enter, as every observable will also carry process-specific factors of $C_{i}$ depending on the fields and vertices involved.

Removing $\left\{\hat{\alpha}, \hat{m}_{Z}^{2}, \hat{G}_{F}\right\}$, we are left with four observables: $\left\{\hat{m}_{W}^{2}, \hat{\Gamma}_{Z \nu_{L} \bar{\nu}_{L}}, \hat{\Gamma}_{Z e_{L} \bar{e}_{L}}, \hat{\Gamma}_{Z e \bar{e}}\right\}$. To make it easier to spot and quantify the effects from SMEFT, we swap out $\hat{m}_{W}^{2}$ for the Veltman $\hat{\rho}$, and divide all partial widths by their SM values:

$$
\begin{gathered}
\hat{\rho} \equiv \frac{\hat{m}_{W}^{2} 2}{\hat{m}_{Z}^{2}} \frac{\hat{x}}{(1-\sqrt{1-\hat{x}}),} \\
\hat{r}_{Z L_{L} \bar{\nu}_{L}} \equiv \frac{24 \pi}{\sqrt{2} \hat{G}_{F} \hat{m}_{Z}^{3}} \hat{\Gamma}_{Z \nu_{L} \bar{\nu}_{L}}, \\
\hat{r}_{Z e_{L} \bar{e}_{L}} \equiv \frac{24 \pi}{\sqrt{2} \hat{G}_{F} \hat{m}_{Z}^{3}(1-\hat{x})} \hat{\Gamma}_{Z e_{L} \bar{e}_{L}}, \\
\hat{r}_{Z e \bar{e}} \equiv \frac{24 \pi}{\sqrt{2} \hat{G}_{F} \hat{m}_{Z}^{3}(1-\sqrt{1-\hat{x}})^{2}} \hat{\Gamma}_{Z e \bar{e}}
\end{gathered}
$$

where we have introduced the convenient combination

\footnotetext{
${ }^{8}$ Discussions of the strengths and weaknesses of the two schemes can be found in Ref. [46].
} 


$$
\hat{x} \equiv \frac{2 \sqrt{2} \pi \hat{\alpha}}{\hat{G}_{F} \hat{m}_{Z}^{2}}, \quad \text { with } \quad \hat{x}_{\mathrm{SM}}=s_{2 \theta}^{2} .
$$

The four observables in Eqs. (27a)-(27d) are unity in SM, but are modified in SMEFT. Because we are only interested in the corrections from SMEFT at the dim-6 level, we only need to keep up to the linear terms in the Wilson coefficients $C_{i}$ (see Table II for definitions of Warsaw basis operators). Assuming universality among fermion generations, we obtain

$$
\begin{gathered}
\hat{\rho}=1+\frac{v^{2}}{c_{2 \theta}}\left[-2 s_{\theta}^{2}\left(\frac{c_{\theta}}{s_{\theta}} C_{H W B}+C_{H l}^{(3)}\right)+\frac{1}{2} s_{\theta}^{2} C_{12}-\frac{1}{2} c_{\theta}^{2} C_{H D}\right], \\
\hat{r}_{Z L_{L} \bar{\nu}_{L}}=1+v^{2}\left[\frac{1}{2} C_{12}-\frac{1}{2} C_{H D}-2 C_{H l}^{(1)}\right], \\
\hat{r}_{Z e_{L} \bar{e}_{L}}=1+\frac{v^{2}}{c_{2 \theta}^{2}}\left[-4 s_{\theta}^{2}\left(\frac{c_{\theta}}{s_{\theta}} C_{H W B}+C_{H l}^{(3)}\right)+\frac{1}{2} C_{12}-\frac{1}{2} C_{H D}+2 c_{2 \theta} C_{H l}^{(1)}\right], \\
\hat{r}_{Z \bar{e} \bar{e}}=1+\frac{v^{2}}{c_{2 \theta}}\left[2\left(\frac{c_{\theta}}{s_{\theta}} C_{H W B}+C_{H l}^{(3)}\right)-\frac{1}{2} C_{12}+\frac{1}{2} C_{H D}-\frac{c_{2 \theta}}{s_{\theta}^{2}} C_{H e}\right] .
\end{gathered}
$$

More details of deriving these results are explained in Appendix A. We have checked that these results agree with Ref. [54] (see also Refs. $[53,55])$. Note that our expression for the Veltman $\hat{\rho}$ in Eq. (29a) reduces to the Peskin-Takeuchi expression in Eq. (1b) in the special case of oblique corrections only where $C_{H l}^{(3)}=C_{12}=0$, upon identifying ${ }^{9}$

$$
\begin{gathered}
\alpha S=2 v^{2} s_{2 \theta} C_{H W B}, \\
\alpha T=-\frac{1}{2} v^{2} C_{H D}, \\
\alpha U=0 .
\end{gathered}
$$

On the other hand, Eqs. (29a)-(29d) hold for general SMEFT in the Warsaw basis. In addition, these results apply to SMEFT and $\nu$ SMEFT alike, since we did not consider observables involving right-handed neutrinos. ${ }^{10}$

\section{Constructing $\mathscr{T}_{l}$ for $(\nu)$ SMEFT to replace Peskin-Takeuchi $T$}

In order to work out a replacement of the $T$ parameter in the $(\nu$ )SMEFT framework that serves to identify hard UV custodial symmetry violation, we rewrite the results in Eqs. (29a)-(29d) into our custodial basis operators given in Table III. This is straightforward, utilizing the translation relations provided in Table VI:

$$
\begin{gathered}
\hat{\rho}=1+\frac{v^{2}}{c_{2 \theta}}\left[2 s_{\theta}^{2}\left(\frac{2 c_{\theta}}{s_{\theta}} a_{H W B}-a_{H l}^{(3)}\right)+\frac{1}{2} s_{\theta}^{2} a_{12}-2 c_{\theta}^{2} a_{H D}\right], \\
\hat{r}_{Z \nu_{L} \bar{\nu}_{L}}=1+v^{2}\left[\frac{1}{2} a_{12}-2 a_{H D}+2 a_{H l}^{(1)}\right], \\
\hat{r}_{Z e_{L} \bar{e}_{L}}=1+\frac{v^{2}}{c_{2 \theta}^{2}}\left[4 s_{\theta}^{2}\left(\frac{2 c_{\theta}}{s_{\theta}} a_{H W B}-a_{H l}^{(3)}\right)+\frac{1}{2} a_{12}-2 a_{H D}-2 c_{2 \theta} a_{H l}^{(1)}\right],
\end{gathered}
$$

\footnotetext{
${ }^{9}$ Note that $S, T, U$ are already linear order in the Wilson coefficients, so the further difference between $\hat{\alpha}_{\mathrm{SM}}$ and $\hat{\alpha}$ in their accompanying coefficients is beyond our SMEFT order. For this reason, we simply write the multiplying factor as $\alpha$. The same holds for our generalization $\mathscr{T}$ to be presented below.

${ }^{10}$ In principle, one could also include the partial width $\hat{\Gamma}_{Z \nu_{R} \nu_{R}}$ in $\nu$ SMEFT. However, we cannot construct a convenient ratio $\hat{r}_{Z \nu_{R} \nu_{R}}$, because $\hat{\Gamma}_{Z \nu_{R} \nu_{R}}$ vanishes in $\nu \mathrm{SM}$. Furthermore, the existence of this partial width also relies on assuming that the mass of the righthanded neutrino is below the electroweak scale.
} 


$$
\hat{r}_{Z \bar{e} \bar{e}}=1+\frac{v^{2}}{c_{2 \theta}}\left[-2\left(\frac{2 c_{\theta}}{s_{\theta}} a_{H W B}-a_{H l}^{(3)}\right)-\frac{1}{2} a_{12}+2 a_{H D}+\frac{c_{2 \theta}}{s_{\theta}^{2}}\left(a_{H l_{R}}^{(1)+}-a_{H l_{R}}^{(1)-}-a_{H l_{R}}^{(3)+}+a_{H l_{R}}^{(3)-}\right)\right] .
$$

In the absence of custodial violation, these observables become

$$
\begin{gathered}
\hat{\rho}-1 \longrightarrow \frac{v^{2}}{c_{2 \theta}}\left[2 s_{\theta}^{2}\left(\frac{2 c_{\theta}}{s_{\theta}} a_{H W B}-a_{H l}^{(3)}\right)+\frac{1}{2} s_{\theta}^{2} a_{12}\right], \\
\hat{r}_{Z L_{L} \bar{\nu}_{L}}-1 \rightarrow v^{2}\left(\frac{1}{2} a_{12}\right), \\
\hat{r}_{Z e_{L} \bar{e}_{L}}-1 \rightarrow \frac{v^{2}}{c_{2 \theta}^{2}}\left[4 s_{\theta}^{2}\left(\frac{2 c_{\theta}}{s_{\theta}} a_{H W B}-a_{H l}^{(3)}\right)+\frac{1}{2} a_{12}\right], \\
\hat{r}_{Z e \bar{e}}-1 \rightarrow \frac{v^{2}}{c_{2 \theta}}\left[-2\left(\frac{2 c_{\theta}}{s_{\theta}} a_{H W B}-a_{H l}^{(3)}\right)-\frac{1}{2} a_{12}-\frac{c_{2 \theta}}{s_{\theta}^{2}} a_{H l_{R}}^{(3)+}\right] .
\end{gathered}
$$

While none of Eqs. (32a)-(32d) vanish in the custodially symmetric limit, the first three observables are governed by only two independent combinations of (custodial-symmetric) Wilson coefficients. Therefore, it is easy to identify the following linear combination that vanishes when there is no custodial violation:

$$
(\hat{\rho}-1)+\frac{1}{2}\left(\hat{r}_{Z \nu_{L} \bar{\nu}_{L}}-1\right)-\frac{1}{2} c_{2 \theta}\left(\hat{r}_{Z e_{L} \bar{e}_{L}}-1\right) \rightarrow 0 .
$$

Therefore, this combined observable can serve as an indicator of our custodial symmetry/violation. Going back to the general $(\nu)$ SMEFT case where custodial violation is present, we see from Eqs. (29a)-(29d) and (31a)-(31d) that this combined observable is given by

$$
\begin{aligned}
(\hat{\rho}-1)+\frac{1}{2}\left(\hat{r}_{Z \nu_{L} \bar{\nu}_{L}}-1\right)-\frac{1}{2} c_{2 \theta}\left(\hat{r}_{Z e_{L} \bar{e}_{L}}-1\right) & =-\frac{1}{2} v^{2}\left[C_{H D}+4 C_{H l}^{(1)}\right] \\
& =-2 v^{2}\left[a_{H D}-a_{H l}^{(1)}\right] \equiv \alpha \mathscr{T}_{l} .
\end{aligned}
$$

We hence define the Wilson coefficients combination in the second line as $\alpha \mathscr{T}_{l}$-a generalization of the PeskinTakeuchi $T$ parameter that is valid for general $(\nu)$ SMEFT (written in the appropriate basis, i.e., Warsaw or our custodial basis). Clearly, in the special case of just oblique corrections, $C_{H l}^{(1)}=0$, our $\alpha \mathscr{T}_{l}$ reduces back to Eq. (30b).

We see from the above that if there is no custodial violation, $a_{H D}=a_{H l}^{(1)}=0$, then $\mathscr{T}_{l}=0$. However, the converse is not true. Custodial violation can conspire to yield a vanishing $\mathscr{T}_{l}$. This is a limitation of our example set of observables chosen in Eq. (24). As we will explain in Sec. IV D, adding more observables does not resolve this issue until we move beyond $2 \rightarrow 2$ fermion experiments.

Our generalization above is named $\mathscr{T}_{l}$ and not just $\mathscr{T}$. This is because, in the presence of nonoblique corrections, one can in fact construct different generalizations of the $T$ parameter with different flavors of fermions. Our construction above used lepton partial widths of the $Z$ boson, and hence the name $\mathscr{T}_{l}$. We will discuss quark generalizations $\mathscr{T}_{q}$ and $\mathscr{T}_{q_{R}}$ in Appendix B.

\section{The role of RPI in SMEFT}

The first three observables in Eqs. (32a)-(32d) depend on three custodial-symmetric Wilson coefficients $a_{H W B}$, $a_{H l}^{(3)}$, and $a_{12}$, so in general one would not expect a linear relation among them like Eq. (33). From this point of view, it seems that we were lucky to have the two Wilson coefficients $a_{H W B}$ and $a_{H l}^{(3)}$ feeding into Eqs. (32a)-(32d) only as a single linear combination,

$$
\left[\frac{2 c_{\theta}}{s_{\theta}} a_{H W B}-a_{H l}^{(3)}\right]=-\left[\frac{c_{\theta}}{s_{\theta}} C_{H W B}+C_{H l}^{(3)}\right] .
$$

In fact, this grouping was inevitable due to an important property of the observables that we consider: the RPI when restricting to observables that only involve $2 \rightarrow 2$ fermion 
experiments [46]. Observables in $2 \rightarrow 2$ fermion experiments do not receive contributions from the following two operators outside the Warsaw basis:

$$
\begin{gathered}
i g_{2}\left(D^{\mu} H\right)^{\dagger} \tau^{a}\left(D^{\nu} H\right) W_{\mu \nu}^{a}, \\
i g_{1}\left(D^{\mu} H\right)^{\dagger}\left(D^{\nu} H\right) B_{\mu \nu} .
\end{gathered}
$$

These two operators are equivalent to two linear combinations of Warsaw basis operators, which are hence two free directions that one can shift the Warsaw basis Wilson coefficients without affecting the $2 \rightarrow 2$ fermion observables. These are known as RPI shifts in SMEFT [46].

In terms of the Wilson coefficients relevant for Eqs. (29a)-(29d), these RPI shifts are

$$
\begin{gathered}
\left(\begin{array}{c}
C_{H W B} \\
C_{H l}^{(3)}
\end{array}\right) \rightarrow\left(\begin{array}{c}
C_{H W B} \\
C_{H l}^{(3)}
\end{array}\right)+\epsilon_{W}\left(\begin{array}{c}
-\tan \theta \\
1
\end{array}\right), \\
\left(\begin{array}{c}
C_{H W B} \\
C_{H D} \\
C_{H l}^{(1)} \\
C_{H e}
\end{array}\right) \rightarrow\left(\begin{array}{c}
C_{H W B} \\
C_{H D} \\
C_{H l}^{(1)} \\
C_{H e}
\end{array}\right)+\epsilon_{B}\left(\begin{array}{c}
\cot \theta \\
-4 \\
1 \\
2
\end{array}\right),
\end{gathered}
$$

where $\epsilon_{W}$ and $\epsilon_{B}$ are arbitrary coefficients. The first shift above is especially strong, as there are only two Wilson coefficients involved. Staring at Eq. (37a), one can see that the only RPI combination of $C_{H W B}$ and $C_{H l}^{(3)}$ is what appears in Eq. (35). This explains why each of our observable's dependence on $C_{H W B}$ in Eqs. (29a)-(29d) arises as this RPI combination.

Of course, one can also check that each of our observable's expression in Eqs. (29a)-(29d) satisfies the second RPI above [Eq. (37b)] as well. In fact, if one were to solve the six unknown Wilson coefficients in Eqs. (29a)-(29d) from the four equations given measured values of the four observables, one would find precisely Eqs. (37a)-(37b) as the two undetermined directions. This means that our example set of observables chosen in Eq. (24) saturates the resolving ability of $2 \rightarrow 2$ lepton observables; no undetermined directions remain beyond the RPI shifts. Therefore, adding more $2 \rightarrow 2$ fermion observables to Eqs. (29a)-(29d), such as $W$ decay widths, would not help pin down the Wilson coefficients.

Finally, we emphasize that our $\mathscr{T}_{l}$ is also an RPI combination, because it is constructed in Eq. (34) with RPI observables. This can be readily checked against Eqs. (37a)-(37b). On the other hand, $\mathscr{T}_{l}$ is not the only SMEFT RPI generalization of the Peskin-Takeuchi $T$ parameter; there are other $\mathscr{T}_{f}$ that can be constructed with hadronic widths of the $Z$ boson, as we will show in Appendix B.

\section{E. Experimental measurements of our observables}

We have presented our results in terms of the observables

$$
\left\{\hat{\rho}, \hat{\Gamma}_{Z \nu_{L} \bar{\nu}_{L}}, \hat{\Gamma}_{Z e_{L} \bar{e}_{L}}, \hat{\Gamma}_{Z e \bar{e}}\right\}
$$

and an additional set of hadronic observables in Appendix B. Let us now consider how to extract these observables from experimental measurements:

(1) We need the accurately measured $\hat{\alpha}, \hat{G}_{F}, \hat{m}_{Z}^{2}$ as basic inputs.

(2) The observable $\hat{\rho}$ requires a measurement of $\hat{m}_{W}^{2}$.

(3) The widths $\left\{\hat{\Gamma}_{Z e_{L} \bar{e}_{L}}, \hat{\Gamma}_{Z e \bar{e}}\right\}$ are not directly measured in practice. Instead, we extract them from the measurements on the total partial width $\hat{\Gamma}_{Z e_{L} \bar{e}_{L}}+$ $\hat{\Gamma}_{Z e \bar{e}}$ and the forward-backward asymmetry $\hat{A}_{F B}^{0, e}$. Direct measurements of the angular distributions of $e^{+} e^{-} \rightarrow e^{+} e^{-}$on the $Z$ resonance can determine $\hat{A}_{F B}^{0, e}[1]$.

(4) The partial width into electrons $\hat{\Gamma}_{Z e_{L} \bar{e}_{L}}+\hat{\Gamma}_{Z e \bar{e}}$ is not directly measured either. Instead, one uses measurements of the total rate $e^{+} e^{-} \rightarrow e^{+} e^{-}$on the $Z$ resonance as well as the total width of the $Z$ boson, $\Gamma_{Z}$, determined by separate measurements scanning the line shape of $e^{+} e^{-} \rightarrow$ hadrons [1].

(5) The partial width of $Z$ into neutrinos must be inferred by subtracting the measured contributions of the $Z$ partial widths from the measured total width $\Gamma_{Z}$ [1]. For this presentation, we assume flavor universality and neglect the masses of the quarks and leptons. The $Z$ partial width into neutrinos is

$$
3 \hat{\Gamma}_{Z \nu_{L} \bar{\nu}_{L}}=\Gamma_{Z}-\hat{\Gamma}_{Z l l}-\hat{\Gamma}_{Z q q},
$$

where we emphasize that the observable we have used throughout this paper, $\hat{\Gamma}_{Z \nu_{L} \bar{\nu}_{L}}$, is the width into just one generation of neutrinos, and $\hat{\Gamma}_{Z l l}, \hat{\Gamma}_{Z q q}$ are the measured decay widths of $Z$ into leptons and hadrons, respectively.

We are finally in a position to evaluate Eq. (34) using experimental data on our observables as determined above. If one were to evaluate this expression using experimental measurements matched to just the tree-level relations, one would obtain a sizable numerical difference from zero. This is not surprising, since the Weinberg angle determined from the Veltman $\hat{\rho}$ differs substantially from the Weinberg angle determined from the charged lepton asymmetries [20]. The main source of the discrepancy is the one-loop contribution from the top quark to the $W$ and $Z$ self-energies. Including this loop contribution to the Veltman $\hat{\rho}$ parameter will cause the numerical evaluation of $\mathscr{T}_{l}$ to be nearly 0 within experimental errors. The more important quantity is thus the experimental error, i.e., sensitivity, on $\mathscr{T}_{l}$. This is determined by including the errors on all of the experimental inputs $\hat{\rho}, \hat{r}_{Z \nu_{L} \bar{\nu}_{L}}$, and $\hat{r}_{Z e_{L} \bar{e}_{L}}$. The least well-measured 
observable is $\hat{r}_{Z e_{L} \bar{e}_{L}}$, and thus the experimental error on this quantity dominates the constraint on the custodial-violating contribution $-2 v^{2}\left[a_{H D}-a_{H l}^{(1)}\right]$. We find

$$
-2 v^{2}\left[a_{H D}-a_{H l}^{(1)}\right] \simeq 0 \pm 0.003,
$$

which implies, in the absence of an accidental cancellation,

$$
\Lambda_{C V} \equiv \frac{1}{\sqrt{2\left|a_{H D}-a_{H l}^{(1)}\right|}} \simeq 3.1 \mathrm{TeV} .
$$

This is much smaller than the scale that would be deduced by doing a global fit to $S, T$ parameters under the assumption that the new physics contributes only to oblique corrections $\Lambda_{C V}^{\mathrm{PDG}} \simeq 6.6 \mathrm{TeV}[20] .{ }^{11}$ This simple analysis illustrates that "maximal" custodial violation (tree-level contributions to $\left.a_{H D}-a_{H l}^{(1)}\right)$ is allowed by precision electroweak data with a considerably lower scale of new physics than would be deduced under the assumption of oblique-only contributions.

\section{CUSTODIAL-VIOLATING COMPLICATIONS FROM EOM REDUNDANCIES}

There is an intuitive but crucial assumption underlying our analysis in Sec. IV, as was established in Sec. III:

(i) When the UV sector is custodial symmetric, any EFT operators generated by matching would preserve the identified custodial $S U(2)_{R}$ in the limit $g_{1} \rightarrow 0$, and therefore all of the custodial-violating operators in our custodial basis (Table III) are absent.

Interestingly, this is not completely true. Only the first half of the above statement is true, while the second half can be invalidated by the EOM redundancies in $(\nu)$ SMEFT

After integrating out a custodial-symmetric UV sector, the resulted EFT operators have to be custodial $S U(2)_{R}$-singlets (in the limit $g_{1} \rightarrow 0$ ), but may lie outside of an arbitrarily chosen operator basis. In order to present the entire EFT in the Warsaw/custodial basis, one may need to apply redundancy relations to trade outside operators with linear combinations of Warsaw/custodial basis operators. While IBP and Fierz redundancies do not change the $S U(2)_{R}$-preserving/violating nature of operators, the EOM redundancies may mix operators that preserve the custodial $S U(2)_{R}$ with those that do not, because $(\nu)$ SM Yukawa couplings break it. As a result, the linear combinations traded from outside operators may contain custodial-violating operators in our custodial basis in Table III.

To better illustrate this issue, we take a closer look at a specific example that we will actually encounter later in some of our example UV theories in Sec. VI. Consider the

\footnotetext{
${ }^{11}$ The number was obtained by taking the upper and lower bounds on $T$ from the $90 \% S-T$ ellipse presented in Ref. [20].
}

operator $Q_{R} \equiv|H|^{2}|D H|^{2}$. Upon taking the limit $g_{1} \rightarrow 0$, this operator preserves the global symmetry $S U(2)_{R H}$. Therefore, according to our discussions in Sec. III, it can be possibly generated by custodial-symmetric UV sectors. For example, it is indeed generated at tree level by integrating out a heavy $W_{L}^{\prime}$ gauge boson, as we will see in Sec. VI B. The problem, however, is that $Q_{R}$ does not belong to the Warsaw/ custodial basis; we need to use IBP and Higgs EOM redundancy relations to trade it into Warsaw/custodial basis operators. From the $\nu$ SM Lagrangian given in Eqs. (3) and (4), we obtain the Higgs EOM relation

$$
\begin{aligned}
& H^{\dagger} D^{2} H+\text { H.c. } \\
& =2 \lambda v^{2}|H|^{2}-4 \lambda|H|^{4} \\
& \quad-\left(Y_{u} \bar{q} \tilde{H} u+Y_{d} \bar{q} H d+Y_{\nu} \bar{l} \tilde{H} \nu+Y_{e} \bar{l} H e+\text { H.c. }\right) .
\end{aligned}
$$

Note that in order to make the expression compact, we have multiplied the EOM by $H^{\dagger}$ from the left and also added its Hermitian conjugate. Combining this with IBP, we can convert $Q_{R}$ into

$$
\begin{aligned}
Q_{R} & =|H|^{2}|D H|^{2} \\
& \equiv 2 \lambda Q_{H}+\frac{1}{2} Q_{H \square}+\frac{1}{2} Q_{Y}-\lambda v^{2}|H|^{4},
\end{aligned}
$$

where we have defined the operator combination

$$
\begin{aligned}
Q_{Y} \equiv & Y_{u} Q_{u H}+Y_{d} Q_{d H}+Y_{\nu} Q_{\nu H}+Y_{e} Q_{e H}+\text { H.c. } \\
= & \frac{1}{4}\left[\left(Y_{u}+Y_{d}\right) O_{q H}^{+}+\left(Y_{u}-Y_{d}\right) O_{q H}^{-}\right. \\
& \left.+\left(Y_{\nu}+Y_{e}\right) O_{l H}^{+}+\left(Y_{\nu}-Y_{e}\right) O_{l H}^{-}\right]+ \text {H.c. }
\end{aligned}
$$

Due to the Yukawa mismatch $Y_{u} \neq Y_{d}$ and $Y_{\nu} \neq Y_{e}$, this combination contains custodial-violating operators $O_{q H}^{-}$and $O_{l H}^{-}$. We see that once traded into the Warsaw/custodial basis, the $S U(2)_{R H}$-preserving operator $Q_{R}$ corresponds to a mixture of custodial-preserving and -violating operators in our custodial basis. This is a consequence of applying Higgs EOM redundancies relations in Eq. (42), which breaks $S U(2)_{R H}$.

\section{A. Robustness of our observables and $\mathscr{T}_{l}$ parameter}

Our observable results given in Eqs. (32a)-(32d) assumed the presence of only custodial-preserving operators in our custodial basis. Now, given that custodialviolating operators could also appear from EOM redundancies, our analysis in Sec. IV is potentially incomplete. In this section, we show that this EOM subtlety does not affect our results in Sec. IV, provided we restrict to tree-level matching.

As was originally worked out in Refs. [47,48] and recently emphasized and generalized in Ref. [49], only a small subset of dim-6 SMEFT operators can be generated 
TABLE I. Custodial $S U(2)_{R}$ invariants outside of the Warsaw basis, which could yield custodial-violating operators in the Warsaw basis upon using $H$ and $\psi$ EOM redundancies.

\begin{tabular}{lcccccc}
\hline \hline Total & $H^{4} D^{2}$ & $\bar{\psi} \psi \psi H^{2} D$ & $H^{2} D^{4}$ & $\bar{\psi} \psi D^{3}$ & $\bar{\psi} \psi X D$ & $\bar{\psi} \psi H D^{2}$ \\
\hline 38 & 1 & 8 & 1 & 4 & 8 & 16 \\
\hline \hline
\end{tabular}

by tree-level matching. ${ }^{12}$ In particular, dim- 6 operators with field strengths $X_{\mu \nu}$ cannot be generated at tree level. This immediately removes the EOMs for gluons, the $W$ boson, and the $B$ boson from consideration. So the only potentially problematic EOMs are those for the Higgs $H$ and the fermions $\psi$. Next, let us find all of the $\nu$ SMEFT dim- 6

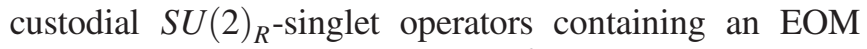
factor of $H$ or $\psi$, i.e., containing $D^{2} H, \not \psi$, or $\not \bar{\psi}$. Using the Hilbert series technique [32-36], with these EOM redundancy relations relaxed, ${ }^{13}$ we find that there are 38 additional real custodial $S U(2)_{R}$ singlets outside of the Warsaw basis. They can be divided into six classes according to the field content, as listed in Table I.

When restricted to tree-level matching, again due to the argument given in Refs. [47-49], only the first two classes in Table I, i.e., $H^{4} D^{2}$ and $\bar{\psi} \psi H^{2} D$, can be generated. Let us examine what custodial-violating operators in our Table III can be obtained from trading these two classes of operators into the Warsaw/custodial basis. They contain nine operators, which are nothing but the $\nu$ SMEFT "kinetic terms" multiplied by $|H|^{2}$ :

$$
\begin{gathered}
|H|^{2}|D H|^{2}, \\
|H|^{2} \bar{\psi} i \not D \psi+\text { H.c. }, \quad \text { with } \quad \psi=q, l, q_{R}, l_{R} .
\end{gathered}
$$

We already analyzed the first operator above, and showed the result of transforming it into the Warsaw basis through EOM in Eqs. (43) and (44). The second operator transformed into the Warsaw basis becomes

$$
|H|^{2} \bar{q} i \not D q=Y_{u} Q_{u H}+Y_{d} Q_{d H}, \quad \text { for } \psi=q,
$$

and similarly for the others. We see that the custodialviolating operators obtained through this procedure are all in "class 5" of Table II: $\bar{\psi} \psi H^{3}$. However, it is clear from Eqs. (29a)-(29d) that none of these operators would feed into the observables discussed in Sec. IV, even when they are present. ${ }^{14}$ Therefore, our results in Eqs. (32a)-(32d) stand, and hence the subsequent analysis presented in

\footnotetext{
${ }^{12}$ Note that this argument is not limited to the Warsaw basis operator set.

${ }^{13}$ This can be achieved by taking $H, \psi$, and $\bar{\psi}$ (and their descendants) as "long representations" of the conformal group, as opposed to "short representations." See Ref. [36] for details.

${ }^{14}$ Recall that we have neglected the fermion masses in the decay widths.
}

Sec. IV, provided that we limit ourselves to treelevel matching. ${ }^{15}$ For example, let us rearrange the EOM relation in Eq. (43) as

$$
Q_{Y}=2 Q_{R}-4 \lambda Q_{H}-Q_{H \square}+2 \lambda v^{2}|H|^{4} .
$$

When restricted to $Z$-pole observables and also neglecting the fermion masses as in Sec. IV, the lhs does not contribute, and so neither does the rhs. Therefore, the combination on the rhs is a free direction that can be viewed as a new set of RPI shifts among $S U(2)_{R}$ singlets. In this language, when the outside operator $Q_{R}$ is generated, one can use this new RPI shift to trade it for other $S U(2)_{R}$ singlets, which will then be in our Warsaw/ custodial basis.

\section{APPLICATION TO UV THEORIES WITH CUSTODIAL SYMMETRY/VIOLATION}

In this section, we examine several UV theories and demonstrate that our $\mathscr{T}_{l}$ parameter is sensitive to (hard) custodial symmetry/violation. We consider in Sec. VI A a real triplet scalar; in Sec. VI B a heavy $W_{L}^{\prime}$ from embedding $S U(2)_{L}$ into $S U(2)_{A} \times S U(2)_{B}$; in Sec. VIC a heavy $Z^{\prime}$ from a spontaneously broken $U(1)_{B-L}$ theory; in Sec. VI D heavy $W^{\prime}$ 's and $Z^{\prime}$ 's from embedding the electroweak group into $S U(2)_{L} \times S U(2)_{R} \times U(1)_{B-L}$; and finally in Sec. VIE two heavy vector-like fermions transforming as $S U(2)_{L}$ singlets. Several highlights of the lessons that we learn from these UV examples are as follows:

(1) Our $\mathscr{T}_{l}$ parameter works perfectly for all of these examples. When the UV sector is custodial symmetric or violating, our $\mathscr{T}_{l}$ is zero or nonzero accordingly.

(2) The heavy $W_{L}^{\prime}$ example in Sec. VI B reminds us that the Veltman $\hat{\rho}$ can possibly deviate from unity in the case of custodial-symmetric UV physics.

(3) The vector-like fermions theory discussed in Sec. VIE serves as a striking example that our new $\mathscr{T}_{l}$ parameter captures nonoblique custodial violation of the UV theory while, unsurprisingly, the PeskinTakeuchi $T$ parameter fails to do so.

\section{A. Triplet scalar extension}

The first UV example we consider is the well-studied SM extension by a real $S U(2)_{L}$-triplet scalar $\phi^{a}$; see, e.g., Refs. [56-61]. The most general renormalizable Lagrangian for this model is

\footnotetext{
${ }^{15}$ Amusingly, the argument here can also be recast into a (new) reparametrization-invariance relation among $S U(2)_{R}$-singlet operators, in the same spirit as that in Ref. [46] (see our discussion in Sec. IV D).
} 


$$
\mathcal{L}_{\mathrm{UV}}=\mathcal{L}_{\mathrm{SM}}+\frac{1}{2}\left(D^{\mu} \phi^{a}\right)\left(D_{\mu} \phi^{a}\right)-\frac{1}{2} M^{2} \phi^{a} \phi^{a}-A H^{\dagger} t^{a} H \phi^{a}-\kappa|H|^{2} \phi^{a} \phi^{a}-\lambda_{\phi}\left(\phi^{a} \phi^{a}\right)^{2} .
$$

This UV theory has (hard) custodial violation due to the interaction term $H^{\dagger} t^{a} H \phi^{a}$. It is well known that this custodial violation shows up already at tree level in the EFTİn what follows below, we check that our new $\mathscr{T}_{l}$ parameter captures this effect.

Integrating out $\phi^{a}$ at tree level, we obtain a SMEFT up to dim-6,

$$
\begin{aligned}
\mathcal{L}_{\mathrm{SMEFT}} & =\mathcal{L}_{\mathrm{SM}}+\frac{A^{2}}{8 M^{2}}|H|^{4}-\frac{\kappa A^{2}}{4 M^{4}} Q_{H}-\frac{A^{2}}{2 M^{4}}\left(\frac{1}{4} Q_{H \square}+Q_{H D}-Q_{R}\right) \\
& =\mathcal{L}_{\mathrm{SM}}+\frac{A^{2}}{8 M^{2}}\left(1-\frac{4 \lambda v^{2}}{M^{2}}\right)|H|^{4}-\frac{A^{2}}{4 M^{4}}(\kappa-4 \lambda) Q_{H}-\frac{A^{2}}{2 M^{4}}\left(-\frac{1}{4} Q_{H \square}+Q_{H D}-\frac{1}{2} Q_{Y}\right) .
\end{aligned}
$$

As expected, the custodial-violating operator $Q_{H D}$ is generated. We also see the appearance of the operator $Q_{R}$ which is outside of the Warsaw basis. In the second line, we have traded it into combinations of Warsaw basis operators using Eq. (43), and hence obtained an additional custodial-violating operator $Q_{Y}$ [see Eq. (44) for the definition]. Reading off the Warsaw basis Wilson coefficients $C_{i}$ from the above and translating to our custodial basis $a_{i}$ using Table $\mathrm{V}$, we obtain

$$
\begin{gathered}
a_{H}=-\frac{A^{2}}{32 M^{4}}(\kappa-4 \lambda), \\
a_{H \square}=\frac{A^{2}}{4 M^{4}}, \\
a_{H D}=-\frac{A^{2}}{8 M^{4}}, \\
a_{l H}^{ \pm}=\frac{A^{2}}{16 M^{4}}\left(Y_{\nu} \pm Y_{e}\right), \\
a_{q H}^{ \pm}=\frac{A^{2}}{16 M^{4}}\left(Y_{u} \pm Y_{d}\right) .
\end{gathered}
$$

Note that in addition to the $a_{H D}$, the "class 5" (see Table III) custodial-violating operators $a_{l H}^{-}$and $a_{q H}^{-}$also show up due to the EOM subtlety discussed in Sec. V, but as explained in Sec. VA, they do not invalidate our analysis.

Now using our definition in Eq. (34), we obtain

$$
\alpha \mathscr{T}_{l}=-2 v^{2}\left[a_{H D}-a_{H l}^{(1)}\right]=\frac{v^{2} A^{2}}{4 M^{4}} \neq 0 .
$$

We see that our $\mathscr{T}_{l}$ parameter captures the hard custodial violation. Since there is no vertex correction in this example [see the first line of Eq. (48)], our $\mathscr{T}_{l}$ reduces to the PeskinTakeuchi $T$ parameter as explained before. So they work equally well in this case.

\section{B. A heavy $W_{L}^{\prime}$ gauge boson}

In this section, we consider a UV theory of embedding the $S U(2)_{L}$ of the $S M$ into $S U(2)_{A} \times S U(2)_{B}$. Specifically, the gauge sector of the UV Lagrangian is

$$
\begin{aligned}
\mathcal{L}_{\mathrm{UV}} \supset & -\frac{1}{4} W_{A \mu \nu}^{a} W_{A}^{a \mu \nu}-\frac{1}{4} W_{B \mu \nu}^{a} W_{B}^{a \mu \nu} \\
& +\frac{1}{2} \operatorname{tr}\left[\left(D^{\mu} \Phi\right)^{\dagger}\left(D_{\mu} \Phi\right)\right]-V_{\Phi},
\end{aligned}
$$

where the heavy scalar field $\Phi$ is a $2 \times 2$ matrix that transforms as a bifundamental under $\left(U_{A}, U_{B}\right) \in$ $S U(2)_{A} \times S U(2)_{B}$ :

$$
\Phi \rightarrow U_{A} \Phi U_{B}^{\dagger}
$$

Therefore, the concrete form of its covariant derivative is

$$
D_{\mu} \Phi=\partial_{\mu} \Phi-i g_{A} W_{A \mu}^{a} t^{a} \Phi+i g_{B} \Phi W_{B \mu}^{a} t^{a},
$$

where $t^{a}=\frac{1}{2} \sigma^{a}$ are the $S U(2)$ generators in the fundamental representation.

The symmetry $S U(2)_{A} \times S U(2)_{B}$ is spontaneously broken by the VEV of the heavy scalar field:

$$
\Phi \supset \frac{v_{\Phi}}{\sqrt{2}}\left(\begin{array}{ll}
1 & 0 \\
0 & 1
\end{array}\right) .
$$

The unbroken group is the diagonal $S U(2)$ formed by the generators $t_{A}^{a}+t_{B}^{a}$, which we identify as our $S U(2)_{L}$ group in the SM. The corresponding gauge boson is the $W$ boson. For the broken generators, the corresponding gauge boson $W_{L}^{\prime}$ acquires mass from $v_{\Phi}$ :

$$
\begin{aligned}
\frac{1}{2} \operatorname{tr} & {\left[\left(D^{\mu} \Phi\right)^{\dagger}\left(D_{\mu} \Phi\right)\right] } \\
& \supset \frac{1}{8} v_{\Phi}^{2}\left(g_{A} W_{A}^{a \mu}-g_{B} W_{B}^{a \mu}\right)\left(g_{A} W_{A \mu}^{a}-g_{B} W_{B \mu}^{a}\right) \\
\quad= & \frac{1}{8} v_{\Phi}^{2}\left(g_{A}^{2}+g_{B}^{2}\right) W_{L}^{\prime a \mu} W_{L \mu}^{\prime a} .
\end{aligned}
$$


We see that $m_{W_{L}^{\prime}}^{2}=\frac{1}{4}\left(g_{A}^{2}+g_{B}^{2}\right) v_{\Phi}^{2}$, and

$$
\begin{aligned}
W_{L \mu}^{\prime a} & \equiv \frac{1}{\sqrt{g_{A}^{2}+g_{B}^{2}}}\left(g_{A} W_{A \mu}^{a}-g_{B} W_{B \mu}^{a}\right), \\
W_{\mu}^{a} & \equiv \frac{1}{\sqrt{g_{A}^{2}+g_{B}^{2}}}\left(g_{B} W_{A \mu}^{a}+g_{A} W_{B \mu}^{a}\right) .
\end{aligned}
$$

With the above rotation, we can rewrite the general covariant derivative as

$$
\begin{aligned}
D_{\mu}= & \partial_{\mu}-i g_{A} W_{A \mu}^{a} t_{A}^{a}-i g_{B} W_{B \mu}^{a} t_{B}^{a} \\
= & \partial_{\mu}-i g_{2} W_{\mu}^{a}\left(t_{A}^{a}+t_{B}^{a}\right) \\
& -i W_{L \mu}^{\prime a}\left(\frac{g_{A}^{2}}{\sqrt{g_{A}^{2}+g_{B}^{2}}} t_{A}^{a}-\frac{g_{B}^{2}}{\sqrt{g_{A}^{2}+g_{B}^{2}}} t_{B}^{a}\right),
\end{aligned}
$$

with the SM gauge coupling $g_{2}=\frac{g_{A} g_{B}}{\sqrt{g_{A}^{2}+g_{B}^{2}}}$ identified.

For the UV interactions between the gauge sector in Eq. (51) and the SM fields, we assume that $W_{A}$ plays the role of $W$ before the symmetry breaking, namely, that the SM fields couple to $W_{A}$ exactly the way they couple to the $W$ boson in SM, and do not couple to $W_{B}$ at all. ${ }^{16}$ This means that for nontrivially $S U(2)_{L}$-charged $\mathrm{SM}$ fields, $t_{A}^{a} \neq 0$ but $t_{B}^{a}=0$. From Eq. (57), we see that after the symmetry breaking, the SM fields couple to both $W$ and $W_{L}^{\prime}$.

In the following, we will match this UV theory with SMEFT by integrating out the heavy $W_{L}^{\prime}$ gauge boson at the tree level. As is clear from the setup, the UV interactions in this example respect the symmetry $S U(2)_{R H}$ [as well as the other $S U(2)_{R}$ symmetries discussed in Sec. II], and hence are custodial symmetric by our definition. We therefore expect a vanishing $\mathscr{T}_{l}$ in the resulting EFT.

Up to linear power in $W_{L}^{\prime}$, the UV interaction is

$$
\mathcal{L}_{\mathrm{UV}} \supset \frac{g_{A}^{2}}{\sqrt{g_{A}^{2}+g_{B}^{2}}} W_{L \mu}^{\prime a} J_{W}^{a \mu},
$$

where $J_{W}^{a \mu}$ denotes the SM $S U(2)_{L}$ current:

$$
J_{W \mu}^{a}=\frac{1}{2}\left(H^{\dagger} i \stackrel{\leftrightarrow}{D_{\mathrm{SM}, \mu}^{a}} H+\sum_{\psi} \bar{\psi} \gamma_{\mu} \tau^{a} \psi\right) .
$$

Integrating out $W_{L}^{\prime}$ at tree level, we obtain a SMEFT up to dim- 6 as

$$
\begin{aligned}
\mathcal{L}_{\text {SMEFT }} & =-\frac{g_{A}^{4}}{g_{A}^{2}+g_{B}^{2}} \frac{1}{2 m_{W_{L}^{\prime}}^{2}} J_{W \mu}^{a} J_{W}^{a \mu} \\
& =-\frac{2 c_{A}^{4}}{v_{\Phi}^{2}} J_{W \mu}^{a} J_{W}^{a \mu},
\end{aligned}
$$

where we have defined the mixing angle $c_{A} \equiv \frac{g_{A}}{\sqrt{g_{A}^{2}+g_{B}}}$. Clearly, this EFT Lagrangian preserves $S U(2)_{R H}$. Plugging in Eq. (59), we obtain

$$
\begin{aligned}
\mathcal{L}_{\mathrm{SMEFT}}= & -\frac{c_{A}^{4}}{v_{\Phi}^{2}}\left[\frac{1}{2} Q_{R}+\frac{1}{8} Q_{H \square}+Q_{H l}^{(3)}+Q_{H q}^{(3)}+\frac{1}{2} Q_{l l}+\frac{1}{2} Q_{q q}^{(3)}+Q_{l q}^{(3)}\right] \\
= & -\frac{c_{A}^{4}}{v_{\Phi}^{2}}\left[\lambda Q_{H}+\frac{3}{8} Q_{H \square}+\frac{1}{4}\left(Y_{u} Q_{u H}+Y_{d} Q_{d H}+Y_{\nu} Q_{\nu H}+Y_{e} Q_{e H}+\text { H.c. }\right)\right. \\
& \left.+Q_{H l}^{(3)}+Q_{H q}^{(3)}+\frac{1}{2} Q_{l l}+\frac{1}{2} Q_{q q}^{(3)}+Q_{l q}^{(3)}\right] .
\end{aligned}
$$

From the first line above, we see that all of the effective operators are $S U(2)_{R H}$ preserving, as expected from Eq. (60). However, in the second line, the $S U(2)_{R H^{-}}$ breaking operator $Q_{Y}$ [see Eq. (44) for the definition] shows up, due to trading $Q_{R}$ for operators in the Warsaw basis using Eq. (43). Reading off the Warsaw basis Wilson coefficients $C_{i}$ from the above and translating to our custodial basis $a_{i}$ using Table $\mathrm{V}$, we obtain

\footnotetext{
${ }^{16}$ While this is the simplest coupling scheme, it is also possible to split the left-handed fermion generations between coupling to $W_{A}$ and coupling to $W_{B}$. In this case, after the $S U(2)_{A} \times S U(2)_{B} \rightarrow$ $S U(2)_{L}$ breaking all left-handed fermions will couple as usual to $W_{L}$ but the interactions with $W_{L}^{\prime}$ will be flavor dependent.
}

$$
\begin{aligned}
& \left\{\begin{array} { l } 
{ a _ { H } = - \frac { c _ { A } ^ { 4 } } { v _ { \Phi } ^ { 2 } } \frac { 1 } { 8 } \lambda } \\
{ a _ { H \square } = - \frac { c _ { A } ^ { 4 } } { v _ { \Phi } ^ { 2 } } \frac { 3 } { 8 } }
\end{array} \left\{\begin{array}{l}
a_{l H}^{ \pm}=-\frac{c_{A}^{4}}{v_{\Phi}^{2}} \frac{1}{16}\left(Y_{\nu} \pm Y_{e}\right), \\
a_{q H}^{ \pm}=-\frac{c_{A}^{4}}{v_{\Phi}^{2}} \frac{1}{16}\left(Y_{u} \pm Y_{d}\right),
\end{array}\right.\right. \\
& a_{H l}^{(3)}=a_{H q}^{(3)}=2 a_{l l}=2 a_{q q}^{(3)}=a_{l q}^{(3)}=-\frac{c_{A}^{4}}{v_{\Phi}^{2}} .
\end{aligned}
$$

Again, we find the appearance of the "class 5" (see Table III) custodial-violating operators $a_{l H}^{-}$and $a_{q H}^{-}$, as expected from the EOM subtlety discussed in Sec. V. Nevertheless, they do not invalidate our analysis because they do not feed into our observables discussed in Sec. IV, as we explained in Sec. VA. 
From the Wilson coefficients above and Eq. (34), it is straightforward to see that $\mathscr{T}_{l}$ vanishes,

$$
\alpha \mathscr{T}_{l}=-2 v^{2}\left[a_{H D}-a_{H l}^{(1)}\right]=0,
$$

which demonstrates the consistency with the UV physics being custodial symmetric.

As a side note, this example also reminds us that the Veltman $\hat{\rho}$ can deviate from 1 in the presence of custodialsymmetric UV physics. ${ }^{17}$ To see this point, we can compute $\hat{\rho}$ with Eqs. (31a)-(31d). However, we first need to extract the $a_{12}$ [defined in Eq. (23)] from the $a_{l l}$ result above. To do so, we restore the generation indices in $Q_{l l}$ from Eq. (60):

$$
\mathcal{L}_{\text {SMEFT }} \supset-\frac{c_{A}^{4}}{2 v_{\Phi}^{2}} \sum_{p, r=1}^{3}\left(\bar{l}_{p} \gamma_{\mu} \tau^{a} l_{p}\right)\left(\bar{l}_{r} \gamma^{\mu} \tau^{a} l_{r}\right) \text {. }
$$

To make this into the form of $Q_{p r s t}$, we need to also restore the $S U(2)_{L}$ indices being contracted, and use the group identity:

$$
\tau_{i j}^{a} \tau_{k l}^{a}=4\left(\frac{1}{2} \delta_{i l} \delta_{j k}-\frac{1}{4} \delta_{i j} \delta_{k l}\right)
$$

Substituting this in, we get

$$
\begin{aligned}
\mathcal{L}_{\mathrm{SMEFT}} & \supset-\frac{c_{A}^{4}}{2 v_{\Phi}^{2}} \sum_{p, r=1}^{3}\left(\bar{l}_{p}^{i} \gamma_{\mu} \tau_{i j}^{a} l_{p}^{j}\right)\left(\bar{l}_{r}^{k} \gamma^{\mu} \tau_{k l}^{a} l_{r}^{l}\right) \\
& =-\frac{c_{A}^{4}}{2 v_{\Phi}^{2}} \sum_{p, r=1}^{3}\left[2\left(\bar{l}_{p}^{i} \gamma_{\mu} l_{p}^{j}\right)\left(\bar{l}_{r}^{j} \gamma^{\mu} l_{r}^{i}\right)-\left(\bar{l}_{p}^{i} \gamma_{\mu} l_{p}^{i}\right)\left(\bar{l}_{r}^{j} \gamma^{\mu} l_{r}^{j}\right)\right] \\
& =-\frac{c_{A}^{4}}{2 v_{\Phi}^{2}} \sum_{p, r=1}^{3}\left[2\left(\bar{l}_{p} \gamma_{\mu} l_{r}\right)\left(\bar{l}_{r} \gamma^{\mu} l_{p}\right)-\left(\bar{l}_{p} \gamma_{\mu} l_{p}\right)\left(\bar{l}_{r} \gamma^{\mu} l_{r}\right)\right] .
\end{aligned}
$$

To obtain the last line above, we have used the Fierz identity for the first term in the square brackets, and then suppressed the $S U(2)_{L}$ indices as usual. Now we can read off the Wilson coefficient with generation indices:

$$
\underset{p p r s t}{a_{p l}}=-\frac{c_{A}^{4}}{2 v_{\Phi}^{2}}\left(2 \delta_{p t} \delta_{r s}-\delta_{p r} \delta_{s t}\right)
$$

Now from Eq. (23) we get

$$
a_{12}=a_{1221}+a_{2112}=-2 \frac{c_{A}^{4}}{v_{\Phi}^{2}} .
$$

\footnotetext{
${ }^{17}$ This issue is unfortunately quite confusing in Ref. [20], which suggests that $\hat{\rho} \neq 1$ implies custodial symmetry violation, which is not correct in general.
}

Plugging all of the relevant Wilson coefficients into Eqs. (31a)-(31d), we obtain $\hat{\rho}$ as

$$
\begin{aligned}
\hat{\rho} & =1+\frac{v^{2}}{c_{2 \theta}}\left[2 s_{\theta}^{2}\left(\frac{2 c_{\theta}}{s_{\theta}} a_{H W B}-a_{H l}^{(3)}\right)+\frac{1}{2} s_{\theta}^{2} a_{12}-2 c_{\theta}^{2} a_{H D}\right] \\
& =1+\frac{s_{\theta}^{2}}{c_{2 \theta}} \frac{c_{A}^{4} v^{2}}{v_{\Phi}^{2}} \neq 1 .
\end{aligned}
$$

Note that $\hat{\rho} \neq 1$ in this example is from the nonoblique corrections $a_{\mathrm{Hl}}^{(3)}$ and $a_{12}$. On the other hand, $a_{H D}=a_{H W B}=0$, so a naive implementation of Eqs. (1b) and (30a)-(30c) would misleadingly predict that the Veltman $\hat{\rho}=1$. This highlights one limitation of the oblique framework (although this is not about custodial symmetry).

In fact, the UV theory in this example is actually a "universal theory," in the sense that one can find an operator basis (different from the Warsaw/custodial basis) in which all of the effective operators are oblique corrections. Concretely, the SMEFT Lagrangian we obtained in Eq. (60) can be fully written into a single effective operator $\left(D^{\mu} W_{\mu \nu}^{a}\right)^{2}$ by using the SM $W$ boson EOM, which is then obviously oblique (only contributing to the two-point function of the $W$ boson). However, even in the case of a universal theory, finding the desired basis and working out the oblique parameters in that basis requires additional effort, and must again be done on a case-by-case basis. On the other hand, restricting to the Warsaw basis and accommodating the nonoblique corrections provides a more systematic approach.

\section{A heavy $Z^{\prime}$ associated with the $U(1)_{B-L}$ symmetry}

In this section, we consider a UV model with a heavy $Z^{\prime}$ gauge boson, associated with the $U(1)_{B-L}$ symmetry in SM (see, e.g., Ref. [62]). This classical symmetry can be broken at the quantum level through triangle anomalies. To consistently gauge the symmetry, one has to ensure that the triangle anomaly contributions from different fermion species are canceled. This can be simply achieved by introducing three SM-singlet right-handed neutrinos $\nu$, a requirement that is satisfied automatically by $\nu \mathrm{SM}$ and $\nu$ SMEFT.

Assuming that this $U(1)_{B-L}$ gauge boson $Z^{\prime}$ couples to the $B-L$ current $j_{B-L} \equiv j_{B}-j_{L}$ through a coupling $\frac{1}{2} g_{Z}$, our UV Lagrangian is ${ }^{18}$

\footnotetext{
${ }^{18}$ In principle, our $U(1)_{B-L}$ gauge boson $Z_{B-L}^{\prime}$ can also mix with the hypercharge gauge boson $B$ through a coupling $\frac{1}{2} \epsilon B^{\mu \nu} Z_{\mu \nu}^{\prime}$. We set this coupling to zero for simplicity in this UV theory example. This is legitimate in our analysis as we only focus on the tree-level matching and neglect radiative effects.
} 


$$
\begin{aligned}
\mathcal{L}_{\mathrm{UV}}= & \mathcal{L}_{\mathrm{SM}}-\frac{1}{4} Z_{\mu \nu}^{\prime} Z^{\prime \mu \nu}+\frac{1}{2} M^{2} Z_{\mu}^{\prime} Z^{\prime \mu} \\
& +g_{\mathrm{Z}} Z_{\mu}^{\prime} \sum_{\psi=q, u, d, l, l, e} \bar{\psi} \gamma^{\mu} \mathrm{y}_{\psi}^{\prime} \psi
\end{aligned}
$$

Here the specific values of the charge $\mathrm{y}_{\psi}^{\prime}=\frac{1}{2}(B-L)$ are

$$
\begin{gathered}
\mathrm{y}_{q}^{\prime}=\mathrm{y}_{u}^{\prime}=\mathrm{y}_{d}^{\prime}=\frac{1}{6} \equiv \mathrm{y}_{1}^{\prime} \quad \text { for quarks, } \\
\mathrm{y}_{l}^{\prime}=\mathrm{y}_{\nu}^{\prime}=\mathrm{y}_{e}^{\prime}=-\frac{1}{2} \equiv \mathrm{y}_{2}^{\prime} \text { for leptons. }
\end{gathered}
$$

We have also assumed that the $Z^{\prime}$ has a large mass $M \gg v$. This can be acquired through the Higgsing from a heavy scalar in the UV which only couples to $Z^{\prime}$, or via a Stueckelberg mechanism which allows $M$ to be a free parameter in the model.

This example is trivially custodial symmetric by our definition, because the UV interactions do not involve the SM Higgs, and hence the $S U(2)_{R H}$ symmetry is trivially preserved. The $\nu$ SMEFT side of the story is similarly trivial. Only operators not involving the Higgs field can be generated at the tree level (the four-fermion operators in this case, as we will see); they are custodial-preserving operators due to trivially respecting $S U(2)_{R H}$. In particular, no custodial-violating operators can be possibly generated by this example, so we can already get $\mathscr{T}_{l}=0$ without carrying out the matching calculation.

Nevertheless, this example is still interesting, because apart from the custodial symmetry [which must involve $S U(2)_{R H}$; see Sec. II D], our custodial basis also helps make manifest the operator structure under the isospin symmetries $S U(2)_{R q_{R}}$ and $S U(2)_{R l_{R}}$. The UV interactions in Eq. (70) with the charges given in Eqs. (71a)-(71b) clearly also preserve these two isospin symmetries. Below, we will check that no isospin-violating operators will be generated in the resulting $\nu$ SMEFT.

Integrating out the $Z^{\prime}$ at tree level, we obtain the $\nu$ SMEFT Lagrangian

$\mathcal{L}_{\nu \mathrm{SMEFT}}=-\frac{g_{Z}^{2}}{2 M^{2}}\left(\sum_{f} \bar{\psi} \gamma_{\mu} \mathrm{y}_{\psi}^{\prime} \psi\right)\left(\sum_{f} \bar{\psi} \gamma^{\mu} \mathrm{y}_{\psi}^{\prime} \psi\right)$,

where $f=\{q, u, d, l, \nu, e\}$. We see that only four-fermion operators of the type $(\bar{L} L)(\bar{L} L),(\bar{R} R)(\bar{R} R)$, and $(\bar{L} L)(\bar{R} R)$ are generated. In the Warsaw basis, the Wilson coefficients can be summarized as

$$
C_{u d}^{(1)}=C_{q u}^{(1)}=C_{q d}^{(1)}=2 C_{q q}^{(1)}=2 C_{u u}=2 C_{d d}=-\frac{g_{Z}^{2}}{2 M^{2}} 2\left(\mathrm{y}_{1}^{\prime}\right)^{2},
$$

$$
\begin{gathered}
C_{\nu e}=C_{l \nu}=C_{l e}=2 C_{l l}=2 C_{\nu \nu}=2 C_{e e}=-\frac{g_{Z}^{2}}{2 M^{2}} 2\left(\mathrm{y}_{2}^{\prime}\right)^{2}, \\
C_{l q}^{(1)}=C_{\nu u}=C_{\nu d}=C_{e u}=C_{e d}=C_{l u}=C_{l d} \\
=C_{q \nu}=C_{q e}=-\frac{g_{Z}^{2}}{2 M^{2}} 2 \mathrm{y}_{1}^{\prime} \mathrm{y}_{2}^{\prime} .
\end{gathered}
$$

Transforming to our custodial basis defined in Table III (again by applying the dictionary in Table V), we see that the only nonzero Wilson coefficients are those preserving both of the isospin symmetries $S U(2)_{R q_{R}} \times S U(2)_{R l_{R}}$ :

$$
\left\{\begin{array} { l } 
{ a _ { l l } = - \frac { g _ { Z } ^ { 2 } } { 2 M ^ { 2 } } ( \mathrm { y } _ { 2 } ^ { \prime } ) ^ { 2 } } \\
{ a _ { q q } ^ { ( 1 ) } = - \frac { g _ { Z } ^ { 2 } } { 2 M ^ { 2 } } ( \mathrm { y } _ { 1 } ^ { \prime } ) ^ { 2 } } \\
{ a _ { l q } ^ { ( 1 ) } = - \frac { g _ { Z } ^ { 2 } } { 2 M ^ { 2 } } 2 \mathrm { y } _ { 1 } ^ { \prime } \mathrm { y } _ { 2 } ^ { \prime } }
\end{array} \left\{\begin{array} { l } 
{ a _ { l _ { R } l _ { R } } ^ { + + } = - \frac { g _ { Z } ^ { 2 } } { 2 M ^ { 2 } } ( \mathrm { y } _ { 2 } ^ { \prime } ) ^ { 2 } } \\
{ a _ { q _ { R } q _ { R } } ^ { ( 1 ) + + } = - \frac { g _ { Z } ^ { 2 } } { 2 M ^ { 2 } } ( \mathrm { y } _ { 1 } ^ { \prime } ) ^ { 2 } } \\
{ a _ { l _ { R } q _ { R } } ^ { ( 1 ) + + } = - \frac { g _ { Z } ^ { 2 } } { 2 M ^ { 2 } } 2 \mathrm { y } _ { 1 } ^ { \prime } \mathrm { y } _ { 2 } ^ { \prime } }
\end{array} \left\{\begin{array}{c}
a_{l l_{R}}^{+}=-\frac{g_{Z}^{2}}{2 M^{2}} 2\left(\mathrm{y}_{2}^{\prime}\right)^{2} \\
a_{l q_{R}}^{+}=-\frac{g_{Z}^{2}}{2 M^{2}} 2 \mathrm{y}_{1}^{\prime} \mathrm{y}_{2}^{\prime} \\
a_{q l_{R}}^{+}=-\frac{g_{Z}^{2}}{2 M^{2}} 2 \mathrm{y}_{1}^{\prime} \mathrm{y}_{2}^{\prime} \\
a_{q q_{R}}^{(1)+}=-\frac{g_{Z}^{2}}{2 M^{2}} 2\left(\mathrm{y}_{1}^{\prime}\right)^{2}
\end{array}\right.\right.\right.
$$

No isospin-violating operators in our Table III are generated, consistent with what we expected from the UV physics.

\section{Heavy $W$ 's and $Z$ 's from a UV theory with $S U(2)_{L} \times S U(2)_{R} \times U(1)_{B-L}$}

In this section, we consider a simple custodial-symmetric UV embedding of the electroweak sector, by promoting the electroweak gauge symmetry to $S U(2)_{L} \times S U(2)_{R} \times$
$U(1)_{B-L}$, which we hence refer to as the 2-2-1 model. The covariant derivative is now

$$
D_{\mu}=\partial_{\mu}-i g W_{\mu}^{a} t^{a}-i g_{R} R_{\mu}^{a} t_{R}^{a}-i g_{K} \mathrm{y}^{\prime} K_{\mu},
$$

where $R_{\mu}^{a}, K_{\mu}$ are the gauge bosons and $t_{R}^{a}, \mathrm{y}^{\prime}$ are the corresponding generators for $S U(2)_{R}$ and $U(1)_{B-L}$. Note that the gauge coupling $g_{R}$ forces the three different $S U(2)_{R}$ symmetries in $\nu \mathrm{SM}$ to be the same; in other words, it breaks 
them down to the diagonal subgroup of $S U(2)_{R H} \times$ $S U(2)_{R q_{R}} \times S U(2)_{R l_{R}}$.

In order to break the enlarged symmetry $S U(2)_{L} \times$ $S U(2)_{R} \times U(1)_{B-L}$ down to electroweak symmetry at low energy, we introduce a new heavy scalar field $\Phi$, which is an $S U(2)_{R}$ doublet with $\mathrm{y}_{\Phi}^{\prime}=\frac{1}{2}$ and an $S U(2)_{L}$ singlet. Upon acquiring a VEV,

$$
\Phi \supset \frac{1}{\sqrt{2}}\left(\begin{array}{c}
0 \\
v_{\phi}
\end{array}\right)
$$

it breaks $S U(2)_{R} \times U(1)_{B-L}$ to $U(1)_{Y}$, with the hypercharge $\mathrm{y}=t_{R}^{3}+\mathrm{y}^{\prime} .{ }^{19}$ In this example, the custodial symmetry is an exact symmetry respected by the UV theory at the high energy scale. However, it is spontaneously broken at the scale $v_{\phi}$. Once we integrate out the heavy gauge bosons and $\Phi$, this $v_{\phi}$ gives rise to (all) the custodialviolating effects in the resulting SMEFT, putting the hypercharge part of the dim- 4 custodial violations and those at higher mass dimensions on the same footing. This is in analogy with the case of minimal flavor violation [63]. ${ }^{20}$
The UV sector in this example is

$$
\begin{aligned}
\mathcal{L}_{\mathrm{UV}} \supset & -\frac{1}{4} W_{\mu \nu}^{a} W^{a, \mu \nu}-\frac{1}{4} R_{\mu \nu}^{a} R^{a, \mu \nu}-\frac{1}{4} K_{\mu \nu} K^{\mu \nu} \\
& +|D \Phi|^{2}-V_{\Phi}+|D H|^{2}+\bar{\psi} i D \psi .
\end{aligned}
$$

Here we have switched off any possible interactions between $\Phi$ and $H$ for simplicity, and hence focus on the effects of integrating out the heavy gauge bosons. After the symmetry breaking, we can identify the mass eigenstates of the gauge bosons

$$
\left(R_{\mu}^{a}, K_{\mu}\right) \rightarrow\left(R_{\mu}^{ \pm}, X_{\mu}, B_{\mu}\right),
$$

among which $B_{\mu}$ remains massless, but $R_{\mu}^{ \pm}$and $X_{\mu}$ obtain masses

$$
\begin{gathered}
m_{R}^{2}=\frac{1}{4} g_{R}^{2} v_{\phi}^{2}, \\
m_{X}^{2}=\frac{1}{4}\left(g_{R}^{2}+g_{K}^{2}\right) v_{\phi}^{2} .
\end{gathered}
$$

We then integrate out these heavy gauge bosons (together with the heavy scalar $\Phi$ ) at tree level, and obtain the EFT Lagrangian up to dim- 6 ,

$$
\begin{aligned}
\mathcal{L}_{\mathrm{EFT}}= & \mathcal{L}_{\mathrm{SM}}+\frac{g_{R}^{2}}{2 m_{R}^{2}}\left[\left(i D_{\mathrm{SM}}^{\mu} \tilde{H}^{\dagger}\right) H+\sum_{q_{R}, l_{R}} \bar{\psi} \gamma^{\mu} t_{R}^{-} \psi\right]\left[H^{\dagger}\left(i D_{\mathrm{SM}, \mu} \tilde{H}\right)-\sum_{q_{R}, l_{R}} \bar{\psi} \gamma_{\mu} t_{R}^{+} \psi\right] \\
& -\frac{g_{R}^{2}}{2 m_{X}^{2} c_{R}^{2}}\left[\frac{c_{R}^{2}}{2}\left(H^{\dagger} i \stackrel{\leftrightarrow}{\stackrel{D}{D}_{\mathrm{SM}}} H\right)+\sum_{q_{R}, l_{R}} \bar{\psi} \gamma^{\mu}\left(t_{R}^{3}-s_{R}^{2} \mathrm{y}\right) \psi\right]\left[\frac{c_{R}^{2}}{2}\left(H^{\dagger} i \stackrel{\leftrightarrow}{D}_{\mathrm{SM}, \mu} H\right)+\sum_{q_{R}, l_{R}} \bar{\psi} \gamma_{\mu}\left(t_{R}^{3}-s_{R}^{2} \mathrm{y}\right) \psi\right]
\end{aligned}
$$

Here the mixing angle is defined as usual

$$
c_{R}=\cos \theta_{R} \equiv \frac{g_{R}}{\sqrt{g_{R}^{2}+g_{K}^{2}}},
$$

and the SM gauge coupling for hypercharge is recovered as

$$
g_{1}^{2}=\frac{g_{R}^{2} g_{K}^{2}}{g_{R}^{2}+g_{K}^{2}}
$$

We see from the result in Eq. (80) that there are generically custodial-violating operators, such as $Q_{H D}$ appearing in the following combinations:

\footnotetext{
${ }^{19}$ The story is completely in parallel with how the SM Higgs $H$ breaks $S U(2)_{L} \times U(1)_{Y}$ to $U(1)_{\mathrm{EM}}$, with electric charge $Q=t^{3}+\mathrm{y}$.

${ }^{20}$ Note that this example would not account for the Yukawainduced custodial violation in the SM.
}

$$
\begin{aligned}
& {\left[\left(i D_{\mathrm{SM}}^{\mu} \tilde{H}^{\dagger}\right) H\right]\left[H^{\dagger}\left(i D_{\mathrm{SM}, \mu} \tilde{H}\right)\right]=Q_{H D}-Q_{R},} \\
& \left(H^{\dagger} i \stackrel{\leftrightarrow}{D}_{\mathrm{SM}}^{\mu} H\right)\left(H^{\dagger} i \stackrel{\leftrightarrow}{D}_{\mathrm{SM}, \mu} H\right)=Q_{H \square}+4 Q_{H D} .
\end{aligned}
$$

This is simply a reflection of the fact that the $S U(2)_{R}$ is spontaneously broken by $v_{\phi}$. Next, we carry out the standard routine of expanding the EFT Lagrangian, trading operators outside of our desired basis (such as $Q_{R}$ above) into the Warsaw basis, reading off the Wilson coefficients $C_{i}$, and translating them into our custodial basis $a_{i}$. The end result contains a large set of Wilson coefficients. The coefficients relevant for computing our observables in Eqs. (31a)-(31d) are

$$
\begin{gathered}
a_{H D}=-\frac{1}{2} a_{H l_{R}}^{(1)-}=\frac{1}{2 v_{\phi}^{2}}\left(1-c_{R}^{4}\right), \\
a_{H l}^{(1)}=a_{H l_{R}}^{(1)+}=\frac{1}{v_{\phi}^{2}} s_{R}^{2} c_{R}^{2},
\end{gathered}
$$




$$
a_{H l_{R}}^{(3)+}=\frac{1}{v_{\phi}^{2}} .
$$

First we notice the appearance of $a_{H l_{R}}^{(3)+}$. It is generated because in the 2-2-1 model, the gauging of $S U(2)_{R}$ reduces the three independent global $S U(2)_{R H} \times S U(2)_{R q_{R}} \times$ $S U(2)_{R l_{R}}$ down to one single gauged $S U(2)_{R}$. In fact, $a_{H q_{R}}^{(3)+}$ and $a_{l_{R} q_{R}}^{(3)++}$ are generated as well, but not listed above as they do not enter into our observables.

Next, a nonzero $a_{H D}$ and $a_{H l}^{(1)}$ indeed means that the UV theory violates custodial symmetry. Plugging Eqs. (85a)(85c) into Eq. (34), our $\mathscr{T}_{l}$ parameter serves as a good indicator of the custodial symmetry/violation:

$$
\alpha \mathscr{T}_{l}=-2 v^{2}\left[a_{H D}-a_{H l}^{(1)}\right]=-\frac{v^{2}}{v_{\phi}^{2}} s_{R}^{4} .
$$

Clearly, $\mathscr{T}_{l}$ is generically nonzero in the 2-2-1 model. However, fixing $g_{1}$ and recalling the relations between $g_{1}$ and the primordial couplings $g_{R}, g_{K}$ in Eqs. (81) and (82), there are two interesting limits: $g_{R} \gg g_{K}$ and $g_{R} \ll g_{K}$.

(1) In the limit $g_{R} \gg g_{K}$, we have $g_{K} \rightarrow g_{1}, g_{R} \gg g_{1}$, and $s_{R} \rightarrow 0$. Then, $\mathscr{T}_{l} \rightarrow 0$ in this limit. More specifically, the behavior of the custodial-violating operators in $\mathscr{T}_{l}$ are $a_{H D}, a_{H l}^{(1)} \rightarrow \frac{g_{1}^{2}}{v_{\phi}^{2} g_{R}^{2}} \rightarrow 0$. They are not only small but also proportional to the SM hypercharge coupling $g_{1}$. This limit is asymptotically custodial symmetric.

(2) In the limit of $g_{R} \ll g_{K}$, we have $g_{R} \rightarrow g_{1}, g_{K} \gg g_{1}$, and $s_{R} \rightarrow 1$. We see that $\alpha \mathscr{T}_{l} \rightarrow-\frac{v^{2}}{v_{\phi}^{2}}$ approaching its maximum size allowed. More specifically, the Wilson coefficient $a_{H l}^{(1)} \rightarrow \frac{g_{1}^{2}}{v_{\phi}^{2} g_{K}^{2}} \rightarrow 0$, while $a_{H D} \rightarrow$ $\frac{1}{2 v_{\phi}^{2}} \neq 0$. One may naively think that this limit ought to work precisely as the $Z_{B-L}^{\prime}$ boson model discussed in Sec. VIC. However, this is not the case. Although $g_{R}$ is small compared to $g_{K}$, it has not been completely switched off, and the EFT does not necessarily imply a light $m_{R}$, because $g_{1} v_{\phi}$ should be viewed as parametrically larger compared to the electroweak scale (as $v_{\phi} \gg v$ ). So in this limit, we actually decouple $m_{X}$ instead of $m_{R}$, resulting in a custodial-violating UV theory, as indicated by the nonzero $\mathscr{T}_{l}$ parameter.

\section{E. Heavy vector-like fermions}

In this section, we illustrate an example of integrating out a UV sector with heavy vector-like fermions that interact with the Standard Model charged leptons and neutrinos [64-66]. Such a UV model does not belong to the category of universal theories $[24,42,43]$, and thus the oblique assumption is not admissible. Below, we will see explicitly that the Peskin-Takeuchi $T$ parameter fails to detect the hard custodial violation in the UV interactions, while our new $\mathscr{T}_{l}$ parameter works perfectly.

Consider a UV model with two vector-like fermions $N$ and $E$ that are $\mathrm{SM} S U(2)_{L}$ singlets. They share a common mass $M \gg v$ and interact with the SM in the same way as the $\nu$ SM right-handed leptons $\nu$ and $e$,

$$
\begin{aligned}
\mathcal{L}_{\mathrm{UV}}= & \mathcal{L}_{\mathrm{SM}}+\bar{N}(i \not D-M) N+\bar{E}(i \not D-M) E \\
& -\left(Y_{N} \bar{l} \tilde{H} N+Y_{E} \bar{l} H E+\text { H.c. }\right) .
\end{aligned}
$$

The new UV Yukawa interactions can be rewritten following the same method shown in Eq. (15):

$$
Y_{N} \bar{l} \tilde{H} N+Y_{E} \bar{l} H E=\bar{l} \Sigma\left(\begin{array}{cc}
Y_{N} & 0 \\
0 & Y_{E}
\end{array}\right)\left(\begin{array}{l}
N \\
E
\end{array}\right) \text {. }
$$

We see that if $\left|Y_{N}\right|=\left|Y_{E}\right|$, the $S U(2)_{R H}$ symmetry can be preserved by the UV sector ${ }^{21}$ in the limit $g_{1} \rightarrow 0(N$ and $E$ have different hypercharges). In this case, the UV sector is custodial symmetric. Otherwise, it has hard custodial violation. Let us now check if our $\mathscr{T}_{l}$ parameter can distinguish these two scenarios.

Integrating the heavy vector-like fermions out at tree level, we obtain a SMEFT Lagrangian at dim-6 as ${ }^{22}$

$$
\begin{aligned}
\mathcal{L}_{\text {SMEFT }} \supset & \left(Y_{N} \bar{l} \tilde{H}\right) \frac{i D}{M^{2}}\left(Y_{N}^{*} \tilde{H}^{\dagger} l\right) \\
& +\left(Y_{E} \bar{l} H\right) \frac{i \not D}{M^{2}}\left(Y_{E}^{*} H^{\dagger} l\right) .
\end{aligned}
$$

Expanding this SMEFT Lagrangian and trading operators into the Warsaw and custodial basis, we obtain the Wilson coefficients

$$
\begin{gathered}
a_{H l}^{(3)}=-\frac{1}{4 M^{2}}\left(\left|Y_{N}\right|^{2}+\left|Y_{E}\right|^{2}\right), \\
a_{H l}^{(1)}=-\frac{1}{4 M^{2}}\left(\left|Y_{N}\right|^{2}-\left|Y_{E}\right|^{2}\right), \\
a_{l H}^{ \pm}=\frac{1}{8 M^{2}}\left(Y_{\nu}\left|Y_{N}\right|^{2} \pm Y_{e}\left|Y_{E}\right|^{2}\right) .
\end{gathered}
$$

Plugging these into Eq. (34), we get

$$
\begin{aligned}
\alpha \mathscr{T}_{l} & =-2 v^{2}\left[a_{H D}-a_{H l}^{(1)}\right] \\
& =-\frac{v^{2}}{2 M^{2}}\left(\left|Y_{N}\right|^{2}-\left|Y_{E}\right|^{2}\right) .
\end{aligned}
$$

\footnotetext{
${ }^{21}$ The phase mismatch between $Y_{N}$ and $Y_{E}$ can be absorbed by redefining the field $N$ or $E$.

${ }^{22}$ As is well known, the dim-5 "neutrino mass" operator is also generated by this UV theory, but it is irrelevant for our current discussion.
} 
We see that indeed our $\mathscr{T}_{l}$ parameter vanishes only if $\left|Y_{N}\right|=\left|Y_{E}\right|$, and does not vanish in general. Thus, $\mathscr{T}_{l}$ serves as a perfect indicator of the UV custodial violation. In addition, we notice that in this example the SMEFT framework captures the UV custodial violation through the Wilson coefficient $a_{H l}^{(1)}$, a nonoblique correction, while $a_{H D}=0$. Therefore, the Peskin-Takeuchi $T$ parameter fails to capture custodial violation in this UV theory. Explicitly,

$$
\alpha T=-\frac{1}{2} v^{2} C_{H D}=-2 v^{2} a_{H D}=0 .
$$

This example demonstrates the utility of our new $\mathscr{T}_{l}$ parameter for indicating both oblique and nonoblique custodial violation arising in $(\nu)$ SMEFT.

\section{DISCUSSION}

We have investigated how to faithfully detect hard custodial symmetry/violation in the UV physics beyond the SM, where hard refers to violations that persist in the limit of vanishing $U(1)_{Y}$ gauge coupling $g_{1} \rightarrow 0$. Working with dim-6 $(\nu)$ SMEFT, we introduced a new basis - the custodial basis - which is simply a rewriting of the Warsaw basis operators to make manifest the symmetric/breaking structures of the various $S U(2)_{R}$ symmetries in $(\nu) \mathrm{SM}$. This custodial basis facilitates the recognition of operators that can/cannot be generated at tree level by custodial-symmetric UV physics. With the help of electroweak precision observables, we then identified several example RPI combinations of dim-6 SMEFT Wilson coefficients $\mathscr{T}_{l}$ (as well as $\mathscr{T}_{q}$ and $\mathscr{T}_{q_{R}}$, in Appendix B) that serve as generalizations of the Peskin-Takeuchi $T$ parameter to accommodate nonoblique corrections from general UV physics.

Given measurements of $\hat{\alpha}, \hat{G}_{F}, \hat{m}_{Z}^{2}$, we showed that the electroweak precision observables

$$
\left\{\hat{\rho}, \hat{r}_{Z \nu_{L} \bar{\nu}_{L}}, \hat{r}_{Z e_{L} \bar{e}_{L}}\right\}
$$

can be used to construct $\mathscr{T}_{l}$ [see Eq. (34)]:

$$
\begin{aligned}
& (\hat{\rho}-1)+\frac{1}{2}\left(\hat{r}_{Z \nu_{L} \bar{\nu}_{L}}-1\right)-\frac{c_{2 \theta}}{2}\left(\hat{r}_{Z e_{L} \bar{e}_{L}}-1\right) \\
& =-\frac{1}{2} v^{2}\left[C_{H D}+4 C_{H l}^{(1)}\right] \\
& =-2 v^{2}\left[a_{H D}-a_{H l}^{(1)}\right] \\
& \equiv \alpha \mathscr{T}_{l} .
\end{aligned}
$$

The measurement $\mathscr{T}_{l} \neq 0$ implies that the UV sector violates custodial symmetry at tree level. Importantly, the converse is not true: $\mathscr{T}_{l}=0$ does not immediately imply no custodial violation in the UV sector. There are several exceptions that we have highlighted throughout the paper. For example, our observable has been demonstrated to capture just hard breaking of custodial symmetry, and is not sensitive to soft custodial violations arising from the gauging of hypercharge. In addition, $\mathscr{T}_{l}$ is unable to rule out the accidental cancellation $a_{H D}=a_{H l}^{(1)}$. Furthermore, we have emphasized in Sec. V that $\mathscr{T}_{l}$ is not sensitive to all custodial-violating $(\nu)$ SMEFT operators, e.g., it is not sensitive to $O_{l H}^{-}$or $O_{q H}^{-}$. As we argued in Sec. V, this is a good thing since they may be faked by the EOM redundancy in rewriting custodial-symmetric operators outside our custodial basis. Finally, our $\mathscr{T}_{l}$ is also not sensitive to custodial violation that appears only at loop level at leading matching order. Here we should distinguish between two possibilities: there are well-known loop corrections to our observables purely from the SM physics, such as the contribution to $\hat{\rho}$ from the custodial-violating difference between the top and bottom quark Yukawa couplings. These effects could be incorporated into the framework by redefining our observables to include the SM loop effects (e.g., the Particle Data Group provides a prescription to do this for the Veltman $\rho$ parameter [20]). However, additional contributions to our observables that arise from radiative corrections from $(\nu)$ SMEFT operators are not included. For some theories, radiative corrections are known, such as the singlet scalar model $[67,68]$. In future work we will investigate if there are persistent patterns that belie a UV theory with custodial symmetry even after radiative corrections are included. This may be more conveniently accomplished by changing to the input electroweak parameter set $\hat{G}_{F}, \hat{m}_{Z}^{2}, \hat{m}_{W}^{2}$, to simplify the Wilson coefficient dependence in loop calculations.

Following the same logic used to construct $\mathscr{T}_{l}$, one can use the hadronic pseudo-observables discussed in Appendix B to construct two additional parameters, $\mathscr{T}_{q}$ and $\mathscr{T}_{q_{R}}$ [see Eqs. (B5) and (B6)]:

$$
\begin{gathered}
(\hat{\rho}-1)-\frac{3-4 s_{\theta}^{2}}{2}\left(\hat{r}_{Z u_{L} \bar{u}_{L}}-1\right)+\frac{3-2 s_{\theta}^{2}}{2}\left(\hat{r}_{Z d_{L} \bar{d}_{L}}-1\right) \\
=-\frac{1}{2} v^{2}\left[C_{H D}-12 C_{H q}^{(1)}\right] \\
=-2 v^{2}\left[a_{H D}+3 a_{H q}^{(1)}\right] \\
\equiv \alpha \mathscr{T}_{q}, \\
(\hat{\rho}-1)+2 s_{\theta}^{2}\left(\hat{r}_{Z u \bar{u}}-1\right)-s_{\theta}^{2}\left(\hat{r}_{Z d \bar{d}}-1\right) \\
=-\frac{1}{2} v^{2}\left[C_{H D}-6\left(C_{H u}+C_{H d}\right)\right] \\
=-2 v^{2}\left[a_{H D}+3 a_{H q_{R}}^{(1)+}+3 a_{H q_{R}}^{(3)-}\right] \\
\equiv \alpha \mathscr{T}_{q_{R}} .
\end{gathered}
$$

For the first generation, of course we cannot separately measure the partial widths into left-handed or right-handed up and down quarks. If we were to assume flavor universality, one could use the measurements of the forward-backward asymmetry of bottom quarks and charm quarks [1] combined with the partial widths into (separately) bottom and charm quarks to construct $\mathscr{T}_{q}$ and $\mathscr{T}_{q_{R}}$. However, the measurements of the quark partial widths and asymmetries are somewhat weaker than the lepton partial widths and asymmetries, and so we expect the actual 
constraints from $\mathscr{T}_{q}$ and $\mathscr{T}_{q_{R}}$ to be weaker than $\mathscr{T}_{l}$ that we focused on in the body of the paper.

We demonstrated the viability and usefulness of our results by calculating $\mathscr{T}_{l}$ for several example UV theories. In some cases, the result is trivial. For example, for the heavy $Z^{\prime}$ associated with $U(1)_{B-L}$ [Sec. VIC], the prediction is $\mathscr{T}_{l}=0$, and more specifically $\hat{\rho}=\hat{r}_{Z L_{L} \bar{\nu}_{L}}=\hat{r}_{Z e_{L} \bar{e}_{L}}=$ $\hat{r}_{Z e \bar{e}}=1$. By itself, this is uninformative, since predicting that these observables do not deviate from unity is indistinguishable from the SM. However, when combined with other observables that deviate from the SM prediction, e.g., a new/modified four-fermion interaction, measuring $\mathscr{T}_{l}$ consistent with zero provides evidence that the UV physics is custodial symmetric and consistent with a $U(1)_{B-L}$ interpretation. Similar arguments applies to the heavy $W_{L}^{\prime}$ boson example [Sec. VI B], which is also custodial symmetric. In addition, this example also reminded us that the Veltman $\hat{\rho} \neq 1$ is possible for custodial-symmetric UV physics.

We also considered UV sectors that (generically) possess hard custodial violations. In the real $S U(2)_{L}$-triplet scalar example [Sec. VI A], our $\mathscr{T}_{l}$ works exactly the same as the Peskin-Takeuchi $T$ parameter. Both parameters indicate the presence of hard custodial violation in the UV sector. In the 22-1 example [Sec. VI D], we embedded the SM into a larger gauge symmetry, $S U(2)_{L} \times S U(2)_{R} \times U(1)_{B-L}$. In this case, the gauging of all three global symmetries $S U(2)_{R H} \times$ $S U(2)_{R q_{R}} \times S U(2)_{R l_{R}}$ marries them into one single $S U(2)_{R}$. Then, the spontaneous breaking of $S U(2)_{R} \times U(1)_{B-L} \rightarrow$ $U(1)_{Y}$ down to the SM generically leads to hard custodial violation, where we found that $\mathscr{T}_{l}$ is in general nonzero. Finally, the heavy vector-like fermions [Sec. VIE] are a striking example where the UV physics is not a "universal" theory and requires our replacement parameter $\mathscr{T}_{l}$. Hard custodial violation feeds into SMEFT via the nonoblique correction $a_{l H}^{(1)}$ (but not $a_{H D}$ ). As a result, the PeskinTakeuchi $T$ parameter fails to detect it, but our new $\mathscr{T}_{l}$ parameter works perfectly.

In this paper, we have assumed flavor universality in constructing our $\mathscr{T}_{f}$ parameters. One can certainly generalize our analysis in Sec. IV and Appendix B to include flavordependent deviations to the observables. This would allow for a construction of flavor-sensitive $\mathscr{T}_{f}$ parameters, which could be used for probing nontrivial flavor structure in the UV custodial violation. The SM Yukawa couplings are examples of flavor-dependent custodial violation. Unlike the hypercharge coupling, they have no direct linkage with a general UV sector. However, for UV theories with "minimal flavor violation" [63], couplings in the UV sector are proportional to (powers of) the SM Yukawa couplings. Such UV sectors are necessarily custodial violating (as well as flavor violating). Nevertheless, with the aforementioned generalization, our $\mathscr{T}_{f}$ parameters are capable of capturing this custodial violation, provided that custodial-violating dim-6 operators (beyond those in "class 5" of our Table III) are generated at tree level.

\section{ACKNOWLEDGMENTS}

We thank Spencer Chang for very helpful discussions as this work was being completed. The work of G. D. K., X. L., and T. T. was supported in part by the U.S. Department of Energy under Grant No. DE-SC0011640. The work of A. M. was supported in part by the National Science Foundation under Grant No. PHY-1820860.

\section{APPENDIX A: DETAILS OF MAPPING ONTO OBSERVABLES}

In this Appendix we provide some details on the intermediate steps that lead to our results in Eqs. (29a)(29d). We work with the Warsaw basis of dim-6 $\nu$ SMEFT shown in Table II, assuming flavor universality. We perform tree-level mapping, and only up to dim-6.

First, we find the corrections to the two-point functions of electroweak gauge bosons,

$$
\Pi_{W W}\left(p^{2}\right)=2 p^{2} v^{2} C_{H W},
$$

$$
\begin{aligned}
\Pi_{Z Z}\left(p^{2}\right)= & \frac{1}{2} \hat{m}_{Z, \mathrm{SM}}^{2} v^{2} C_{H D} \\
& +2 p^{2} v^{2}\left(c_{\theta}^{2} C_{H W}+s_{\theta}^{2} C_{H B}+c_{\theta} s_{\theta} C_{H W B}\right),
\end{aligned}
$$

$\Pi_{\gamma \gamma}\left(p^{2}\right)=2 p^{2} v^{2}\left(s_{\theta}^{2} C_{H W}+c_{\theta}^{2} C_{H B}-c_{\theta} s_{\theta} C_{H W B}\right)$,

$\Pi_{\gamma Z}\left(p^{2}\right)=p^{2} v^{2}\left[2 c_{\theta} s_{\theta}\left(C_{H W}-C_{H B}\right)-\left(c_{\theta}^{2}-s_{\theta}^{2}\right) C_{H W B}\right]$,

where as usual $\Pi_{V V}\left(p^{2}\right)$ denotes the transverse part of the full two-point function of the gauge bosons:

$i \Pi_{V V}^{\mu \nu}\left(p^{2}\right)=i \Pi_{V V}\left(p^{2}\right)\left(\eta^{\mu \nu}-\frac{p^{\mu} p^{\nu}}{p^{2}}\right)+\left(i \frac{p^{\mu} p^{\nu}}{p^{2}}\right.$ term $)$.

Next, we move on to the three-point vertices. For the observables considered in Sec. IV, the relevant vertex corrections between the electroweak gauge bosons and the leptons are

$$
\begin{gathered}
V_{Z \nu_{L} \bar{\nu}_{L}}=1-v^{2}\left[C_{H l}^{(1)}-C_{H l}^{(3)}\right], \\
V_{Z e_{L} \bar{e}_{L}}=1+\frac{v^{2}}{c_{2 \theta}}\left[C_{H l}^{(1)}+C_{H l}^{(3)}\right], \\
V_{Z e \bar{e}}=1-\frac{v^{2}}{2 s_{\theta}^{2}} C_{H e}, \\
V_{W \bar{l}}=1+v^{2} C_{H l}^{(3)} .
\end{gathered}
$$


Note that corrections to the four-fermion vertices would not feed into $\hat{\alpha}$ due to the lack of pole structure. The only fourfermion vertex that needs to be considered in our analysis is $C_{12}$ (as mentioned in Sec. III A), which will feed into $\hat{G}_{F}$.

With the above, we would like to find the modifications to Eqs. (25a)-(25g). The first four observables are relatively simple:

$$
\begin{gathered}
\hat{\alpha}=\frac{g_{1}^{2} g_{2}^{2}}{4 \pi\left(g_{1}^{2}+g_{2}^{2}\right)}\left[\left.\frac{p^{2}}{p^{2}-\Pi_{\gamma \gamma}\left(p^{2}\right)}\right|_{p^{2} \rightarrow 0}\right] \\
=\hat{\alpha}_{\mathrm{SM}}\left[1+2 v^{2}\left(s_{\theta}^{2} C_{H W}+c_{\theta}^{2} C_{H B}-c_{\theta} s_{\theta} C_{H W B}\right)\right], \quad(\mathrm{A} 4 \mathrm{a}) \\
\hat{G}_{F}=\frac{\sqrt{2} g_{2}^{2}}{8} V_{W \bar{l}}^{2}\left[\frac{-1}{\left.p^{2}-\hat{m}_{W, \mathrm{SM}}^{2}-\left.\Pi_{W W}\left(p^{2}\right)\right|_{p^{2} \rightarrow 0}\right]-\frac{1}{2 \sqrt{2}} C_{12}}\right. \\
=\hat{G}_{F, \mathrm{SM}}\left[1+2 v^{2} C_{H l}^{(3)}-\frac{1}{2} v^{2} C_{12}\right], \\
\hat{m}_{Z}^{2}=\hat{m}_{Z, \mathrm{SM}}^{2}+\Pi_{Z Z}\left(\hat{m}_{Z, \mathrm{SM}}^{2}\right) \\
=\hat{m}_{Z, \mathrm{SM}}^{2}\left[1+\frac{1}{2} v^{2} C_{H D}+2 v^{2}\left(c_{\theta}^{2} C_{H W}\right.\right. \\
\left.\left.\quad+s_{\theta}^{2} C_{H B}+c_{\theta} s_{\theta} C_{H W B}\right)\right], \\
\hat{m}_{W}^{2}=\hat{m}_{W, \mathrm{SM}}^{2}+\Pi_{W W}\left(\hat{m}_{W, \mathrm{SM}}^{2}\right)=\hat{m}_{W, \mathrm{SM}}^{2}\left(1+2 v^{2} C_{H W}\right) .
\end{gathered}
$$

These will lead us to the $\hat{\rho}$ expression in Eq. (29a).
For the decay widths in Eqs. (25a)-(25g), we need a bit more setup. We define the amplitude $i \hat{M}$ as the strength $\hat{\kappa}$ multiplied by the polarization kinematics:

$$
i \hat{M}_{Z \psi \bar{\psi} \bar{\psi}} \equiv i \hat{\kappa}\left(\epsilon_{\mu} \bar{u}_{\psi} \gamma^{\mu} P_{L / R} v_{\bar{\psi}}\right),
$$

with $\epsilon_{\mu}$ denoting the polarization vectors for the $Z$ boson, $u$ and $v$ denoting the Dirac spinors for the fermion legs, and $P_{L / R}=\frac{1 \mp \gamma^{5}}{2}$ denoting the projector depending on the chirality of the fermion $\psi$. With this, one can compute the decay width

$$
\hat{\Gamma}_{Z \psi \bar{\psi}}=\frac{1}{16 \pi \hat{m}_{Z}} \overline{\left|\hat{M}_{Z \psi \bar{\psi}}\right|^{2}}=\frac{\hat{m}_{Z}}{24 \pi} \hat{\kappa}^{2}
$$

where fermion masses are neglected. The $\hat{r}$ observables defined in Eqs. (27b)-(27d) can then be expressed as

$$
\begin{gathered}
\hat{r}_{Z L_{L} \bar{\nu}_{L}}=\frac{\hat{\kappa}_{Z \nu_{L} \bar{\nu}_{L}}^{2}}{\sqrt{2} \hat{G}_{F} \hat{m}_{Z}^{2}}, \\
\hat{r}_{Z e_{L} \bar{e}_{L}}=\frac{\hat{\kappa}_{Z e_{L} \bar{e}_{L}}^{2}}{\sqrt{2} \hat{G}_{F} \hat{m}_{Z}^{2}(1-\hat{x})}, \\
\hat{r}_{Z e \bar{e}}=\frac{\hat{\kappa}_{Z e \bar{e}}^{2}}{\sqrt{2} \hat{G}_{F} \hat{m}_{Z}^{2}(1-\sqrt{1-\hat{x}})^{2}},
\end{gathered}
$$

where $\hat{x}$ is defined as before by Eq. (28). In the SM, these ratios are unity. In the SMEFT dim-6 Warsaw basis, the above strengths are modified as

$$
\begin{gathered}
\hat{\kappa}_{Z \nu_{L} \bar{\nu}_{L}}=\hat{\kappa}_{Z \nu_{L} \bar{\nu}_{L}, \mathrm{SM}}\left(R_{Z}\right)^{1 / 2} V_{Z \nu_{L} \bar{\nu}_{L}} \\
=\hat{\kappa}_{Z \nu_{L} \bar{\nu}_{L}, \mathrm{SM}}\left[1+v^{2}\left(c_{\theta}^{2} C_{H W}+s_{\theta}^{2} C_{H B}+c_{\theta} s_{\theta} C_{H W B}\right)-v^{2}\left(C_{H l}^{(1)}-C_{H l}^{(3)}\right)\right], \\
\hat{\kappa}_{Z e_{L} \bar{e}_{L}}=\hat{\kappa}_{Z e_{L} \bar{e}_{L}, \mathrm{SM}}\left(R_{Z}\right)^{1 / 2}\left[V_{Z e_{L} \bar{e}_{L}}+\frac{s_{2 \theta}}{c_{2 \theta}} \frac{1}{p^{2}} \Pi_{\gamma Z}\left(p^{2}\right)\right] \\
=\hat{\kappa}_{Z e_{L} \bar{e}_{L}, \mathrm{SM}}\left[1+v^{2}\left(c_{\theta}^{2} C_{H W}+s_{\theta}^{2} C_{H B}+c_{\theta} s_{\theta} C_{H W B}\right)+v^{2} \frac{1}{c_{2 \theta}}\left(C_{H l}^{(1)}+C_{H l}^{(3)}\right)+v^{2} \frac{s_{2 \theta}^{2}}{c_{2 \theta}}\left(C_{H W}-C_{H B}\right)-2 v^{2} c_{\theta} s_{\theta} C_{H W B}\right], \\
\hat{\kappa}_{Z e \bar{e}}=\hat{\kappa}_{Z e \bar{e}, \mathrm{SM}}\left(R_{Z}\right)^{1 / 2}\left[V_{Z e \bar{e}}-\frac{c_{\theta}}{s_{\theta}} \frac{1}{p^{2}} \Pi_{\gamma Z}\left(p^{2}\right)\right] \\
=\hat{\kappa}_{Z e \bar{e}, \mathrm{SM}}\left[1+v^{2}\left(c_{\theta}^{2} C_{H W}+s_{\theta}^{2} C_{H B}+c_{\theta} s_{\theta} C_{H W B}\right)-v^{2} \frac{1}{2 s_{\theta}^{2}} C_{H e}-v^{2} 2 c_{\theta}^{2}\left(C_{H W}-C_{H B}\right)+v^{2} \frac{c_{\theta}}{s_{\theta}} c_{2 \theta} C_{H W B}\right],
\end{gathered}
$$

where $R_{Z}$ is the residue of the $Z$ boson at the pole mass:

$$
\begin{aligned}
R_{Z} & =1+\left.\left[\frac{d}{d p^{2}} \Pi_{Z Z}\left(p^{2}\right)\right]\right|_{p^{2}=\hat{m}_{Z, S M}^{2}} \\
& =1+2 v^{2}\left(c_{\theta}^{2} C_{H W}+s_{\theta}^{2} C_{H B}+c_{\theta} s_{\theta} C_{H W B}\right) .
\end{aligned}
$$


Plugging Eqs. (A8a)-(A8c) and (A4a)-(A4d) into Eqs. (A7a)-(A7c) will lead us to the expressions for the partial widths in Eqs. (29a)-(29d).

\section{APPENDIX B: HADRONIC PSEUDO-OBSERVABLES}

In this Appendix we consider a set of four-quark partial widths - pseudo-observables — in addition to those listed in Eq. (24):

$$
\left\{\hat{\Gamma}_{Z u_{L} \bar{u}_{L}}, \hat{\Gamma}_{Z d_{L} \bar{d}_{L}}, \hat{\Gamma}_{Z u \bar{u}}, \hat{\Gamma}_{Z d \bar{d}}\right\}
$$

In order, these denote the partial decay widths of the $Z$ boson to left-handed up-type quarks, left-handed downtype quarks, right-handed up-type quarks, and right-handed down-type quarks. Note that in $Z$ decay measurements, the first two generations of quarks are essentially indistinguishable. The measurable observables in practice are $\hat{\Gamma}_{Z q q}$ (which will be needed in measuring $\hat{\Gamma}_{Z_{L} \bar{\nu}_{L}}$; see discussions in Sec. IV E) and measurements involving the $b$ quark. For this reason, we refer to these hadronic partial widths of $Z$ as pseudo-observables to distinguish them from the observables discussed in Sec. IV.

We present our results in terms of definite-parity hadronic final states in order to most easily compare with the results in Sec. IV. In the SM they are given by the three Lagrangian parameters $g_{1}, g_{2}, v$ :

$$
\hat{\Gamma}_{Z u_{L} \bar{u}_{L}, \mathrm{SM}}=\frac{\hat{m}_{Z, \mathrm{SM}}}{288 \pi} \frac{g_{2}^{2}}{c_{\theta}^{2}}\left(3-4 s_{\theta}^{2}\right)^{2},
$$

$$
\begin{gathered}
\hat{\Gamma}_{Z d_{L} \bar{d}_{L}, \mathrm{SM}}=\frac{\hat{m}_{Z, \mathrm{SM}}}{288 \pi} \frac{g_{2}^{2}}{c_{\theta}^{2}}\left(3-2 s_{\theta}^{2}\right)^{2}, \\
\hat{\Gamma}_{Z u \bar{u}, \mathrm{SM}}=\frac{\hat{m}_{Z, \mathrm{SM}}}{18 \pi} \frac{g_{2}^{2}}{c_{\theta}^{2}} s_{\theta}^{4}, \\
\hat{\Gamma}_{Z d \bar{d}, \mathrm{SM}}=\frac{\hat{m}_{Z, \mathrm{SM}}}{72 \pi} \frac{g_{2}^{2}}{c_{\theta}^{2}} s_{\theta}^{4} .
\end{gathered}
$$

We then construct the following ratios [similar to Eqs. (27a)-(27d)] to keep track of the deviations from the SM:

$$
\begin{array}{r}
\hat{r}_{Z u_{L} \bar{u}_{L}} \equiv \frac{72 \pi}{\sqrt{2} \hat{G}_{F} \hat{m}_{Z}^{3}(1+2 \sqrt{1-\hat{x}})^{2}} \hat{\Gamma}_{Z u_{L} \bar{u}_{L}}, \\
\hat{r}_{Z d_{L} \bar{d}_{L}} \equiv \frac{72 \pi}{\sqrt{2} \hat{G}_{F} \hat{m}_{Z}^{3}(2+\sqrt{1-\hat{x}})^{2}} \hat{\Gamma}_{Z d_{L} \bar{d}_{L}}, \\
\hat{r}_{Z u \bar{u}} \equiv \frac{18 \pi}{\sqrt{2} \hat{G}_{F} \hat{m}_{Z}^{3}(1-\sqrt{1-\hat{x}})^{2}} \hat{\Gamma}_{Z u \bar{u}}, \\
\hat{r}_{Z d \bar{d}} \equiv \frac{72 \pi}{\sqrt{2} \hat{G}_{F} \hat{m}_{Z}^{3}(1-\sqrt{1-\hat{x}})^{2}} \hat{\Gamma}_{Z d \bar{d}},
\end{array}
$$

where $\hat{x}$ is defined as before by Eq. (28). These four ratios are unity in the SM, but will get modified in SMEFT. Following the same procedure shown in Appendix A, we obtain their general Warsaw basis corrections as

$\hat{r}_{Z u_{L} \bar{u}_{L}}=1+\frac{v^{2}}{c_{2 \theta}\left(3-4 s_{\theta}^{2}\right)}\left[-8 s_{\theta}^{2}\left(\frac{c_{\theta}}{s_{\theta}} C_{H W B}+C_{H l}^{(3)}-\frac{1}{4} C_{12}\right)-6 c_{2 \theta}\left(C_{H l}^{(3)}-C_{H q}^{(3)}-\frac{1}{4} C_{12}\right)-\frac{1}{2}\left(3-2 s_{\theta}^{2}\right) C_{H D}-6 c_{2 \theta} C_{H q}^{(1)}\right]$,

$$
\begin{gathered}
\hat{r}_{Z d_{L} \bar{d}_{L}}=1+\frac{v^{2}}{c_{2 \theta}\left(3-2 s_{\theta}^{2}\right)}\left[-4 s_{\theta}^{2}\left(\frac{c_{\theta}}{s_{\theta}} C_{H W B}+C_{H l}^{(3)}-\frac{1}{4} C_{12}\right)-6 c_{2 \theta}\left(C_{H l}^{(3)}-C_{H q}^{(3)}-\frac{1}{4} C_{12}\right)\right. \\
\left.-\frac{1}{2}\left(3-4 s_{\theta}^{2}\right) C_{H D}+6 c_{2 \theta} C_{H q}^{(1)}\right], \\
\hat{r}_{Z u \bar{u}}=1+\frac{v^{2}}{c_{2 \theta}}\left[2\left(\frac{c_{\theta}}{s_{\theta}} C_{H W B}+C_{H l}^{(3)}-\frac{1}{4} C_{12}\right)+\frac{1}{2} C_{H D}+\frac{3 c_{2 \theta}}{2 s_{\theta}^{2}} C_{H u}\right], \\
\hat{r}_{Z d \bar{d}}=1+\frac{v^{2}}{c_{2 \theta}}\left[2\left(\frac{c_{\theta}}{s_{\theta}} C_{H W B}+C_{H l}^{(3)}-\frac{1}{4} C_{12}\right)+\frac{1}{2} C_{H D}-\frac{3 c_{2 \theta}}{s_{\theta}^{2}} C_{H d}\right] .
\end{gathered}
$$

Although there are eight Wilson coefficients $C_{i}$ involved in the above, they only come with six different combinations. Furthermore, recall from Eq. (29a) that we have 
$\hat{\rho}=1-\frac{v^{2}}{c_{2 \theta}}\left[2 s_{\theta}^{2}\left(\frac{c_{\theta}}{s_{\theta}} C_{H W B}+C_{H l}^{(3)}-\frac{1}{4} C_{12}\right)+\frac{1}{2} c_{\theta}^{2} C_{H D}\right]$.

We see that in the three quantities $\left\{\hat{\rho}, \hat{r}_{Z u_{L} \bar{u}_{L}}, \hat{r}_{Z d_{L} \bar{d}_{L}}\right\}$, only two independent combinations of custodial-preserving operators show up. Therefore, analogous to the procedure of constructing $\mathscr{T}_{l}$ from Eqs. (32a)-(34), we can construct a new $T$ parameter generalization $\mathscr{T}_{q}$ using $\hat{\rho}$ and the lefthanded partial widths:

$$
\begin{aligned}
(\hat{\rho} & -1)-\frac{3-4 s_{\theta}^{2}}{2}\left(\hat{r}_{Z u_{L} \bar{u}_{L}}-1\right)+\frac{3-2 s_{\theta}^{2}}{2}\left(\hat{r}_{Z d_{L} \bar{d}_{L}}-1\right) \\
& =-\frac{1}{2} v^{2}\left[C_{H D}-12 C_{H q}^{(1)}\right] \\
& =-2 v^{2}\left[a_{H D}+3 a_{H q}^{(1)}\right] \\
& \equiv \alpha \mathscr{T}_{q} .
\end{aligned}
$$

Similarly, a $\mathscr{T}_{q_{R}}$ can be constructed using $\hat{\rho}$ and the righthanded partial widths:

$$
\begin{aligned}
& (\hat{\rho}-1)+2 s_{\theta}^{2}\left(\hat{r}_{Z u \bar{u}}-1\right)-s_{\theta}^{2}\left(\hat{r}_{Z d \bar{d}}-1\right) \\
& =-\frac{1}{2} v^{2}\left[C_{H D}-6\left(C_{H u}+C_{H d}\right)\right] \\
& =-2 v^{2}\left[a_{H D}+3 a_{H q_{R}}^{(1)+}+3 a_{H q_{R}}^{(3)-}\right] \\
& \quad \equiv \alpha \mathscr{T}_{q_{R}} .
\end{aligned}
$$

In the second lines of Eqs. (B5) and (B6), we have used Table VI to write them in terms of our custodial basis Wilson coefficients $a_{i}$, where it becomes manifest that $\mathscr{T}_{q}$ and $\mathscr{T}_{q_{R}}$ receive contributions only from custodial-violating operators in our Table III.

\section{APPENDIX C: TABLES OF OPERATORS, COEFFICIENTS, AND TRANSLATIONS}

In this Appendix we gather tables of operator bases and relevant translation relations. Table II summarizes all of the independent baryon-number-preserving and leptonnumber-preserving operators in the Warsaw basis for

TABLE II. $\nu$ SMEFT dim-6 baryon-number-preserving and lepton-number-preserving operators in the Warsaw basis. In addition to the $76=42+(17+$ H.c. $)$ SMEFT operators, there are $25=7+(9+$ H.c. $)$ new operators involving right-handed neutrinos $\nu$.

\begin{tabular}{ll}
\hline \hline $1: X^{3}$ & \\
\hline$Q_{G}$ & $f^{A B C} G_{\mu}^{A \nu} G_{\nu}^{B \rho} G_{\rho}^{C \mu}$ \\
$Q_{\tilde{G}}$ & $f^{A B C} \tilde{G}_{\mu}^{A \nu} G_{\nu}^{B \rho} G_{\rho}^{C \mu}$ \\
\hline
\end{tabular}

(Table continued)
TABLE II. (Continued)

\begin{tabular}{ll}
\hline \hline $1: X^{3}$ & \\
\hline$Q_{W}$ & $\epsilon^{a b c} W_{\mu}^{a \nu} W_{\nu}^{b \rho} W_{\rho}^{c \mu}$ \\
$Q_{\tilde{W}}$ & $\epsilon^{a b c} \tilde{W}_{\mu}^{a \nu} W_{\nu}^{b \rho} W_{\rho}^{c \mu}$ \\
\hline \hline
\end{tabular}

\begin{tabular}{ll}
\hline \hline $2: H^{6}$ & \\
\hline$Q_{H}$ & $|H|^{6}$
\end{tabular}

3: $H^{4} D^{2}$

\begin{tabular}{lc}
\hline$Q_{H \square}$ & $-\left(\partial_{\mu}|H|^{2}\right)\left(\partial^{\mu}|H|^{2}\right)$ \\
$Q_{H D}$ & {$\left[\left(D_{\mu} H^{\dagger}\right) H\right]\left[H^{\dagger}\left(D^{\mu} H\right)\right]$}
\end{tabular}

\begin{tabular}{lc}
\hline \hline $5: \bar{\psi} \psi H^{3}+$ H.c. & \\
\hline$Q_{\nu H}$ & $|H|^{2}(\bar{l} \tilde{H} \nu)$ \\
$Q_{e H}$ & $|H|^{2}(\bar{l} H e)$ \\
$Q_{u H}$ & $|H|^{2}(\bar{q} \tilde{H} u)$ \\
$Q_{d H}$ & $|H|^{2}(\bar{q} H d)$ \\
\hline \hline
\end{tabular}

\begin{tabular}{lc}
\hline \hline$: X^{2} H^{2}$ & \\
\hline$Q_{H G}$ & $|H|^{2} G_{\mu \nu}^{A} G^{A \mu \nu}$ \\
$Q_{H \tilde{G}}$ & $|H|^{2} \tilde{G}_{\mu \nu}^{A} G^{A \mu \nu}$ \\
$Q_{H W}$ & $|H|^{2} W_{\mu \nu}^{a} W^{a \mu \nu}$ \\
$Q_{H \tilde{W}}$ & $|H|^{2} \tilde{W}_{\mu \nu}^{a} W^{a \mu \nu}$ \\
$Q_{H B}$ & $|H|^{2} B_{\mu \nu} B^{\mu \nu}$ \\
$Q_{H \tilde{B}}$ & $|H|^{2} \tilde{B}_{\mu \nu} B^{\mu \nu}$ \\
$Q_{H W B}$ & $H^{\dagger} \tau^{a} H W_{\mu \nu}^{a} B^{\mu \nu}$ \\
$Q_{H \tilde{W} B}$ & $H^{\dagger} \tau^{a} H \tilde{W}_{\mu \nu}^{a} B^{\mu \nu}$ \\
\hline
\end{tabular}

\begin{tabular}{lc}
\hline \hline 6: $\bar{\psi} \psi X H+$ H.c. & \\
\hline$Q_{\nu W}$ & $\left(\bar{l} \sigma^{\mu \nu} \nu\right) \tau^{a} \tilde{H} W_{\mu \nu}^{a}$ \\
$Q_{e W}$ & $\left(\bar{l} \sigma^{\mu \nu} e\right) \tau^{a} H W_{\mu \nu}^{a}$ \\
$Q_{\nu B}$ & $\left(\bar{l} \sigma^{\mu \nu} \nu\right) \tilde{H} B_{\mu \nu}$ \\
$Q_{e B}$ & $\left(\bar{l} \sigma^{\mu \nu} e\right) H B_{\mu \nu}$ \\
$Q_{u G}$ & $\left(\bar{q} \sigma^{\mu \nu} T^{A} u\right) \tilde{H} G_{\mu \nu}^{A}$ \\
$Q_{d G}$ & $\left(\bar{q} \sigma^{\mu \nu} T^{A} d\right) H G_{\mu \nu}^{A}$ \\
$Q_{u W}$ & $\left(\bar{q} \sigma^{\mu \nu} u\right) \tau^{a} \tilde{H} W_{\mu \nu}^{a}$ \\
$Q_{d W}$ & $\left(\bar{q} \sigma^{\mu \nu} d\right) \tau^{a} H W_{\mu \nu}^{\mu}$ \\
$Q_{u B}$ & $\left(\bar{q} \sigma^{\mu \nu} u\right) \tilde{H} B_{\mu \nu}$ \\
$Q_{d B}$ & $\left(\bar{q} \sigma^{\mu \nu} d\right) H B_{\mu \nu}$ \\
\hline \hline
\end{tabular}

7: $\bar{\psi} \psi H^{2} D$

\begin{tabular}{lc}
\hline$Q_{H l}^{(1)}$ & $\left(H^{\dagger} i \stackrel{\leftrightarrow}{D_{\mu}} H\right)\left(\bar{l} \gamma^{\mu} l\right)$ \\
$Q_{H l}^{(3)}$ & $\left(H^{\dagger} i \stackrel{D_{\mu}^{a}}{\mu} H\right)\left(\bar{l} \gamma^{\mu} \tau^{a} l\right)$ \\
$Q_{H q}^{(1)}$ & $\left(H^{\dagger} i \stackrel{\leftrightarrow}{D_{\mu}} H\right)\left(\bar{q} \gamma^{\mu} q\right)$ \\
$Q_{H q}^{(3)}$ & $\left(H^{\dagger} i \stackrel{\leftrightarrow}{D_{\mu}} H\right)\left(\bar{q} \gamma^{\mu} \tau^{a} q\right)$ \\
$Q_{H \nu}$ & $\left(H^{\dagger} i \stackrel{\leftrightarrow}{D_{\mu}} H\right)\left(\bar{\nu} \gamma^{\mu} \nu\right)$ \\
\hline & (Table continued $)$
\end{tabular}


TABLE II. (Continued)

\begin{tabular}{lc}
\hline \hline $7: \bar{\psi} \psi H^{2} D$ & \\
\hline$Q_{H e}$ & $\left(H^{\dagger} i \stackrel{\leftrightarrow}{D_{\mu} H} H\right)\left(\bar{e} \gamma^{\mu} e\right)$ \\
$Q_{H \nu e}+$ H.c. & $\left(\tilde{H}^{\dagger} i D_{\mu} H\right)\left(\bar{\nu} \gamma^{\mu} e\right)$ \\
$Q_{H u}$ & $\left(H^{\dagger} i \stackrel{\leftrightarrow}{D_{\mu}} H\right)\left(\bar{u} \gamma^{\mu} u\right)$ \\
$Q_{H d}$ & $\left(H^{\dagger} i \stackrel{D}{D}_{\mu} H\right)\left(\bar{d} \gamma^{\mu} d\right)$ \\
$Q_{H u d}+$ H.c. & $\left(\tilde{H}^{\dagger} i D_{\mu} H\right)\left(\bar{u} \gamma^{\mu} d\right)$ \\
\hline \hline $8:(\bar{L} L)(\bar{L} L)$ & \\
\hline$Q_{l l}$ & $\left(\bar{l} \gamma_{\mu} l\right)\left(\bar{l} \gamma^{\mu} l\right)$ \\
$Q_{q q}^{(1)}$ & $\left(\bar{q} \gamma_{\mu} q\right)\left(\bar{q} \gamma^{\mu} q\right)$ \\
$Q_{q q}^{(3)}$ & $\left(\bar{q} \gamma_{\mu} \tau^{a} q\right)\left(\bar{q} \gamma^{\mu} \tau^{a} q\right)$ \\
$Q_{l q}^{(1)}$ & $\left(\bar{l} \gamma_{\mu} l\right)\left(\bar{q} \gamma^{\mu} q\right)$ \\
$Q_{l q}^{(3)}$ & $\left(\bar{l} \gamma_{\mu} \tau^{a} l\right)\left(\bar{q} \gamma^{\mu} \tau^{a} q\right)$ \\
\hline \hline
\end{tabular}

8: $(\bar{R} R)(\bar{R} R)$

\begin{tabular}{lc}
\hline$Q_{\nu \nu}$ & $\left(\bar{\nu} \gamma_{\mu} \nu\right)\left(\overline{\bar{\nu}} \gamma^{\mu} \nu\right)$ \\
$Q_{e e}$ & $\left(\bar{e} \gamma_{\mu} e\right)\left(\bar{e} \gamma^{\mu} e\right)$ \\
$Q_{\nu e}$ & $\left(\bar{\nu} \gamma_{\mu} \nu\right)\left(\bar{e} \gamma^{\mu} e\right)$ \\
$Q_{u u}$ & $\left(\bar{u} \gamma_{\mu} u\right)\left(\bar{u} \gamma^{\mu} u\right)$ \\
$Q_{d d}$ & $\left(\bar{d} \gamma_{\mu} d\right)\left(\bar{d} \gamma^{\mu} d\right)$ \\
$Q_{u d}^{(1)}$ & $\left(\bar{u} \gamma_{\mu} u\right)\left(\bar{d} \gamma^{\mu} d\right)$ \\
$Q_{u d}^{(8)}$ & $\left(\bar{u} \gamma_{\mu} T^{A} u\right)\left(\bar{d} \gamma^{\mu} T^{A} d\right)$ \\
$Q_{\nu u}$ & $\left(\bar{\nu} \gamma_{\mu} \nu\right)\left(\bar{u} \gamma^{\mu} u\right)$ \\
$Q_{\nu d}$ & $\left(\bar{\nu} \gamma_{\mu} \nu\right)\left(\bar{d} \gamma^{\mu} d\right)$ \\
$Q_{e u}$ & $\left(\bar{e} \gamma_{\mu} e\right)\left(\bar{u} \gamma^{\mu} u\right)$ \\
$Q_{e d}$ & $\left(\bar{e} \gamma_{\mu} e\right)\left(\bar{d} \gamma^{\mu} d\right)$ \\
$Q_{\nu e d u}+$ H.c. & $\left(\bar{\nu} \gamma_{\mu} e\right)\left(\bar{d} \gamma^{\mu} u\right)$ \\
\hline
\end{tabular}

8: $(\bar{L} L)(\bar{R} R)$

\begin{tabular}{lc}
\hline$Q_{l \nu}$ & $\left(\bar{l} \gamma_{\mu} l\right)\left(\bar{\nu} \gamma^{\mu} \nu\right)$ \\
$Q_{l e}$ & $\left(\bar{l} \gamma_{\mu} l\right)\left(\bar{e} \gamma^{\mu} e\right)$ \\
$Q_{l u}$ & $\left(\bar{l} \gamma_{\mu} l\right)\left(\bar{u} \gamma^{\mu} u\right)$ \\
$Q_{l d}$ & $\left(\bar{l} \gamma_{\mu} l\right)\left(\bar{d} \gamma^{\mu} d\right)$ \\
$Q_{q \nu}$ & $\left(\bar{q} \gamma_{\mu} q\right)\left(\bar{\nu} \gamma^{\mu} \nu\right)$ \\
$Q_{q e}$ & $\left(\bar{q} \gamma_{\mu} q\right)\left(\bar{e} \gamma^{\mu} e\right)$ \\
$Q_{q u}^{(1)}$ & $\left(\bar{q} \gamma_{\mu} q\right)\left(\bar{u} \gamma^{\mu} u\right)$ \\
$Q_{q d}^{(1)}$ & $\left(\bar{q} \gamma_{\mu} q\right)\left(\bar{d} \gamma^{\mu} d\right)$ \\
$Q_{q u}^{(8)}$ & $\left(\bar{q} \gamma_{\mu} T^{A} q\right)\left(\bar{u} \gamma^{\mu} T^{A} u\right)$ \\
$Q_{q d}^{(8)}$ & $\left(\bar{q} \gamma_{\mu} T^{A} q\right)\left(\bar{d} \gamma^{\mu} T^{A} d\right)$ \\
\hline
\end{tabular}

8: $(\bar{L} R)(\bar{R} L)+$ H.c.

\begin{tabular}{ll}
\hline$Q_{\text {lvuq }}$ & $\left(\bar{l}^{i} \nu\right)\left(\bar{u} q^{i}\right)$ \\
$Q_{\text {ledq }}$ & $\left(\bar{l}^{i} e\right)\left(\bar{d} q^{i}\right)$ \\
\hline \hline
\end{tabular}

8: $(\bar{L} R)(\bar{L} R)+$ H.c.

$Q_{\text {lvle }}$

$\left(\bar{l}^{i} \nu\right) \epsilon_{i j}\left(\bar{l}^{j} e\right)$

(Table continued)
TABLE II. (Continued)

\begin{tabular}{lc}
\hline $8:(\bar{L} R)(\bar{L} R)+$ H.c. & \\
\hline$Q_{q u q d}^{(1)}$ & $\left(\bar{q}^{i} u\right) \epsilon_{i j}\left(\bar{q}^{j} d\right)$ \\
$Q_{q u q d}^{(8)}$ & $\left(\bar{q}^{i} T^{A} u\right) \epsilon_{i j}\left(\bar{q}^{j} T^{A} d\right)$ \\
$Q_{\text {lvqd }}^{(1)}$ & $\left(\bar{l}^{i} \nu\right) \epsilon_{i j}\left(\bar{q}^{j} d\right)$ \\
$Q_{\text {lequ }}^{(1)}$ & $\left(\bar{l}^{i} e\right) \epsilon_{i j}\left(\bar{q}^{j} u\right)$ \\
$Q_{\text {lvqd }}^{(3)}$ & $\left(\bar{l}^{i} \sigma_{\mu \nu} \nu\right) \epsilon_{i j}\left(\bar{q}^{j} \sigma^{\mu \nu} d\right)$ \\
$Q_{\text {lequ }}^{(3)}$ & $\left(\bar{l}^{i} \sigma_{\mu \nu} e\right) \epsilon_{i j}\left(\bar{q}^{j} \sigma^{\mu \nu} u\right)$ \\
\hline \hline
\end{tabular}

TABLE III. $\nu$ SMEFT dim-6 baryon-number-preserving and lepton-number-preserving operators in our custodial basis.

\begin{tabular}{lr}
\hline \hline $1: X^{3}$ & \\
\hline$O_{G}$ & $f^{A B C} G_{\mu}^{A \nu} G_{\nu}^{B \rho} G_{\rho}^{C \mu}$ \\
$O_{\tilde{G}}$ & $f^{A B C} \tilde{G}_{\mu}^{A \nu} G_{\nu}^{B \rho} G_{\rho}^{C \mu}$ \\
$O_{W}$ & $\epsilon^{a b c} W_{\mu}^{a \nu} W_{\nu}^{b \rho} W_{\rho}^{c \mu}$ \\
$O_{\tilde{W}}$ & $\epsilon^{a b c} \tilde{W}_{\mu}^{a \nu} W_{\nu}^{b \rho} W_{\rho}^{c \mu}$ \\
\hline \hline $2: H^{6}$ & {$\left[\operatorname{tr}\left(\Sigma^{\dagger} \Sigma\right)\right]^{3}$} \\
\hline$O_{H}$ & \\
\hline \hline $3: H^{4} D^{2}$ & {$\left[\operatorname{tr}\left(\Sigma^{\dagger} i D_{\mu} \Sigma\right)\right]^{2}$} \\
\hline$O_{H \square}$ & {$\left[\operatorname{tr}\left(\Sigma^{\dagger} i D_{\mu} \Sigma \tau_{R}^{3}\right)\right]^{2}$} \\
$O_{H D}$ & \\
\hline
\end{tabular}

5: $\bar{\psi} \psi H^{3}+$ H.c.

\begin{tabular}{lr}
\hline$O_{l H}^{ \pm}$ & $\operatorname{tr}\left(\Sigma^{\dagger} \Sigma\right)\left(\bar{l} \Sigma P_{ \pm} l_{R}\right)$ \\
$O_{q H}^{ \pm}$ & $\operatorname{tr}\left(\Sigma^{\dagger} \Sigma\right)\left(\bar{q} \Sigma P_{ \pm} q_{R}\right)$ \\
\hline \hline $4: X^{2} H^{2}$ & \\
\hline$O_{H G}$ & $\operatorname{tr}\left(\Sigma^{\dagger} \Sigma\right) G_{\mu \nu}^{A} G^{A \mu \nu}$ \\
$O_{H \tilde{G}}$ & $\operatorname{tr}\left(\Sigma^{\dagger} \Sigma\right) \tilde{G}_{\mu \nu}^{A} G^{A \mu \nu}$ \\
$O_{H W}$ & $\operatorname{tr}\left(\Sigma^{\dagger} \Sigma\right) W_{\mu \nu}^{a} W^{a \mu \nu}$ \\
$O_{H \tilde{W}}$ & $\operatorname{tr}\left(\Sigma^{\dagger} \Sigma\right) \tilde{W}_{\mu \nu}^{a} W^{a \mu \nu}$ \\
$O_{H B}$ & $\operatorname{tr}\left(\Sigma^{\dagger} \Sigma\right) B_{\mu \nu} B^{\mu \nu}$ \\
$O_{H \tilde{B}}$ & $\operatorname{tr}\left(\Sigma^{\dagger} \Sigma\right) \tilde{B}_{\mu \nu} B^{\mu \nu}$ \\
$O_{H W B}$ & $\operatorname{tr}\left(\Sigma^{\dagger} \tau^{a} \Sigma \tau_{R}^{3}\right) W_{\mu \nu}^{a} B^{\mu \nu}$ \\
$O_{H \tilde{W} B}$ & $\operatorname{tr}\left(\Sigma^{\dagger} \tau^{a} \Sigma \tau_{R}^{3}\right) \tilde{W}_{\mu \nu}^{a} B^{\mu \nu}$ \\
\hline
\end{tabular}

\begin{tabular}{lr}
\hline $6: \bar{\psi} \psi X H+$ H.c. \\
\hline$O_{l W}^{ \pm}$ & $\left(\bar{l} \sigma^{\mu \nu} \tau^{a} \Sigma P_{ \pm} l_{R}\right) W_{\mu \nu}^{a}$ \\
$O_{l B}^{ \pm}$ & $\left(\bar{l} \sigma^{\mu \nu} \Sigma P_{\mp} l_{R}\right) B_{\mu \nu}$ \\
$O_{q G}^{ \pm}$ & $\left(\bar{q} \sigma^{\mu \nu} T^{A} \Sigma P_{ \pm} q_{R}\right) G_{\mu \nu}^{A}$ \\
\hline & (Table continued)
\end{tabular}




\section{TABLE III. (Continued)}

\begin{tabular}{lr}
\hline $6: \bar{\psi} \psi X H+$ H.c. & \\
\hline$O_{q W}^{ \pm}$ & $\left(\bar{q} \sigma^{\mu \nu} \tau^{a} \Sigma P_{ \pm} q_{R}\right) W_{\mu \nu}^{a}$ \\
$O_{q B}^{ \pm}$ & $\left(\bar{q} \sigma^{\mu \nu} \Sigma P_{\mp} q_{R}\right) B_{\mu \nu}$ \\
\hline \hline $7: \bar{\psi} \psi H^{2} D$ & \\
\hline$O_{H l}^{(1)}$ & $\operatorname{tr}\left(\Sigma^{\dagger} i D_{\mu} \Sigma \tau_{R}^{3}\right)\left(\bar{l} \gamma^{\mu} l\right)$ \\
$O_{H l}^{(3)}$ & $\operatorname{tr}\left(\Sigma^{\dagger} \tau^{a} i D_{\mu} \Sigma\right)\left(\bar{l} \gamma^{\mu} \tau^{a} l\right)$ \\
$O_{H q}^{(1)}$ & $\operatorname{tr}\left(\Sigma^{\dagger} i D_{\mu} \Sigma \tau_{R}^{3}\right)\left(\bar{q} \gamma^{\mu} q\right)$ \\
$O_{H q}^{(3)}$ & $\operatorname{tr}\left(\Sigma^{\dagger} \tau^{a} i D_{\mu} \Sigma\right)\left(\bar{q} \gamma^{\mu} \tau^{a} q\right)$ \\
$O_{H l_{R}}^{(1) \pm}$ & $\operatorname{tr}\left(\Sigma^{\dagger} i D_{\mu} \Sigma \tau_{R}^{3}\right)\left(\bar{l}_{R} \gamma^{\mu} P_{ \pm} l_{R}\right)$ \\
$O_{H l_{R}}^{(3) \pm}$ & $\operatorname{tr}\left(\Sigma^{\dagger} i D_{\mu} \Sigma \tau_{R}^{a}\right)\left(\bar{l}_{R} \gamma^{\mu} \tau_{R}^{a} P_{ \pm} l_{R}\right)$ \\
$O_{H q_{R}}^{(1) \pm}$ & $\operatorname{tr}\left(\Sigma^{\dagger} i D_{\mu} \Sigma \tau_{R}^{3}\right)\left(\bar{q}_{R} \gamma^{\mu} P_{ \pm} q_{R}\right)$ \\
$O_{H q_{R}}^{(3) \pm}$ & $\operatorname{tr}\left(\Sigma^{\dagger} i D_{\mu} \Sigma \tau_{R}^{a}\right)\left(\bar{q} \gamma_{R} \gamma^{\mu} \tau_{R}^{a} P_{ \pm} q_{R}\right)$ \\
\hline \hline $8:(\bar{L} L)(\bar{L} L)$ & \\
\hline$O_{l l}$ & $\left(\bar{l} \gamma_{\mu} l\right)\left(\bar{l} \gamma^{\mu} l\right)$ \\
$O_{q q}^{(1)}$ & $\left(\bar{q} \gamma_{\mu} q\right)\left(\bar{q} \gamma^{\mu} q\right)$ \\
$O_{q q}^{(3)}$ & $\left(\bar{q} \gamma_{\mu} \tau^{a} q\right)\left(\bar{q} \gamma^{\mu} \tau^{a} q\right)$ \\
$O_{l q}^{(1)}$ & $\left(\bar{l} \gamma_{\mu} l\right)\left(\bar{q} \gamma^{\mu} q\right)$ \\
$O_{l q}^{(3)}$ & $\left(\bar{l} \gamma_{\mu} \tau^{a} l\right)\left(\bar{q} \gamma^{\mu} \tau^{a} q\right)$ \\
\hline \hline
\end{tabular}

TABLE III. (Continued)

8: $(\bar{L} R)(\bar{L} R)+$ H.c.

\begin{tabular}{lc}
\hline$O_{q q_{R} q q_{R}}^{(1)}$ & $\left(\bar{q}^{i} q_{R}^{k}\right) \epsilon_{i j} \epsilon_{k l}\left(\bar{q}^{j} q_{R}^{l}\right)$ \\
$O_{q q_{R} q q_{R}}^{(8)}$ & $\left(\bar{q}^{i} T^{A} q_{R}^{k}\right) \epsilon_{i j} \epsilon_{k l}\left(\bar{q}^{j} T^{A} q_{R}^{l}\right)$ \\
$O_{l l l_{R} q q_{R}}^{(1) \pm}$ & $\left(\bar{l}^{i} l_{R}^{k}\right) \epsilon_{i j}\left(\epsilon P_{ \pm}\right)_{k l}\left(\bar{q}^{j} q_{R}^{l}\right)$ \\
$O_{l l_{R} q q_{R}}^{(3) \pm}$ & $\left(\bar{l}^{i} \sigma_{\mu \nu} l_{R}^{k}\right) \epsilon_{i j}\left(\epsilon P_{ \pm}\right)_{k l}\left(\bar{q}^{j} \sigma^{\mu \nu} q_{R}^{l}\right)$ \\
\hline \hline
\end{tabular}

TABLE IV. A dictionary of the custodial basis operators $O_{i}$ in terms of Warsaw basis operators $Q_{i}$.

\begin{tabular}{lr}
\hline \hline $1: X^{3}$ & \\
\hline$O_{G}$ & $Q_{G}$ \\
$O_{\tilde{G}}$ & $Q_{\tilde{G}}$ \\
$O_{W}$ & $Q_{W}$ \\
$O_{\tilde{W}}$ & $Q_{\tilde{W}}$ \\
\hline \hline $2: H^{6}$ & \\
\hline$O_{H}$ & \\
\hline \hline $3: H^{4} D^{2}$ & $Q_{H}$ \\
\hline$O_{H \square}$ & $Q_{H \square}$ \\
$O_{H D}$ & $Q_{H \square}+4 Q_{H D}$ \\
\hline
\end{tabular}

5: $\bar{\psi} \psi H^{3}+$ H.c.

\begin{tabular}{ll}
\hline$O_{l H}^{ \pm}$ & $2\left(Q_{\nu H} \pm Q_{e H}\right)$ \\
$O_{q H}^{ \pm}$ & $2\left(Q_{u H} \pm Q_{d H}\right)$ \\
\hline \hline
\end{tabular}

4: $X^{2} H^{2}$

\begin{tabular}{lc}
\hline$O_{H G}$ & $2 Q_{H G}$ \\
$O_{H \tilde{G}}$ & $2 Q_{H \tilde{G}}$ \\
$O_{H W}$ & $2 Q_{H W}$ \\
$O_{H \tilde{W}}$ & $2 Q_{H \tilde{W}}$ \\
$O_{H B}$ & $2 Q_{H B}$ \\
$O_{H \tilde{B}}$ & $2 Q_{H \tilde{B}}$ \\
$O_{H W B}$ & $-2 Q_{H W B}$ \\
$O_{H \tilde{W} B}$ & $-2 Q_{H \tilde{W} B}$ \\
\hline \hline
\end{tabular}

6: $\bar{\psi} \psi X H+$ H.c.

\begin{tabular}{ll}
\hline$O_{l W}^{ \pm}$ & $Q_{\nu W} \pm Q_{e W}$ \\
$O_{l B}^{ \pm}$ & $Q_{\nu B} \mp Q_{e B}$ \\
$O_{q G}^{ \pm}$ & $Q_{u G} \pm Q_{d G}$ \\
$O_{q W}^{ \pm}$ & $Q_{u W} \pm Q_{d W}$ \\
$O_{q B}^{ \pm}$ & $Q_{u B} \mp Q_{d B}$
\end{tabular}

\begin{tabular}{ll}
\hline \hline $7: \bar{\psi} \psi H^{2} D$ & \\
\hline$O_{H l}^{(1)}$ & $-Q_{H l}^{(1)}$ \\
\hline
\end{tabular}


TABLE IV. (Continued)

\begin{tabular}{lc}
\hline \hline $7: \bar{\psi} \psi H^{2} D$ & \\
\hline$O_{H l}^{(3)}$ & $Q_{H l}^{(3)}$ \\
$O_{H q}^{(1)}$ & $-Q_{H q}^{(1)}$ \\
$O_{H q}^{(3)}$ & $Q_{H q}^{(3)}$ \\
$O_{H l_{R}}^{(1) \pm}$ & $-\left(Q_{H \nu} \pm Q_{H e}\right)$ \\
$O_{H l_{R}}^{(3) \pm}$ & $\pm 2\left(Q_{H \nu e} \pm\right.$ H.c. $)-Q_{H \nu} \pm Q_{H e}$ \\
$O_{H q_{R}}^{(1) \pm}$ & $-\left(Q_{H u} \pm Q_{H d}\right)$ \\
$O_{H q_{R}}^{(3) \pm}$ & $\pm 2\left(Q_{H u d} \pm\right.$ H.c. $)-Q_{H u} \pm Q_{H d}$ \\
\hline \hline
\end{tabular}

8: $(\bar{L} L)(\bar{L} L)$

$O_{l l}$

$O_{q q}^{(1)}$

$O_{q q}^{(3)}$

$O_{l q}^{(1)}$

$O_{l q}^{(3)}$

=

8: $(\bar{R} R)(\bar{R} R)$

\begin{tabular}{lc}
\hline$O_{l_{R} l_{R}}^{ \pm \pm}$ & $Q_{\nu \nu}+Q_{e e} \pm 2 Q_{\nu e}$ \\
$O_{l_{R} l_{R}}^{+-}$ & $Q_{\nu \nu}-Q_{e e}$ \\
$O_{q_{R} q_{R}}^{(1) \pm \pm}$ & $Q_{u u}+Q_{d d} \pm 2 Q_{u d}^{(1)}$ \\
$O_{q_{R} q_{R}}^{(1)+-}$ & $Q_{u u}-Q_{d d}$ \\
$O_{q_{R} q_{R}}^{(3)++}$ & $8 Q_{u d}^{(8)}-\frac{2 N_{c}-4}{N_{c}} Q_{u d}^{(1)}+Q_{u u}+Q_{d d}$ \\
$O_{l_{R} q_{R}}^{(1) \pm \pm}$ & $\left(Q_{\nu u}+Q_{e d}\right) \pm\left(Q_{\nu d}+Q_{e u}\right)$ \\
$O_{l_{R} q_{R}}^{(1) \pm \mp}$ & $\left(Q_{\nu u}-Q_{e d}\right) \mp\left(Q_{\nu d}-Q_{e u}\right)$ \\
$O_{l_{R} q_{R}}^{(3)+ \pm}$ & $2\left(Q_{\nu e d u} \pm\right.$ H.c. $)+\left(Q_{\nu u}-Q_{e u}\right) \mp\left(Q_{\nu d}-Q_{e d}\right)$ \\
\hline
\end{tabular}

$\overline{\overline{8:(L L)(R R))}}$

8: $(\bar{L} L)(\bar{R} R)$

\begin{tabular}{lc}
\hline$O_{l l_{R}}^{ \pm}$ & $Q_{l \nu} \pm Q_{l e}$ \\
$O_{l q_{R}}^{ \pm}$ & $Q_{l u} \pm Q_{l d}$ \\
$O_{q l_{R}}^{ \pm}$ & $Q_{q \nu} \pm Q_{q e}$ \\
$O_{q q_{R}}^{(1) \pm}$ & $Q_{q u}^{(1)} \pm Q_{q d}^{(1)}$ \\
$O_{q q_{R}}^{(8) \pm}$ & $Q_{q u}^{(8)} \pm Q_{q d}^{(8)}$ \\
\hline \hline
\end{tabular}

8: $(\bar{L} R)(\bar{R} L)+$ H.c.

$\overline{O_{l_{R} q_{R} q}^{ \pm}} \quad Q_{l v u q} \pm Q_{l e d q}$

8: $(\bar{L} R)(\bar{L} R)+$ H.c.

\begin{tabular}{lc}
\hline$O_{l l_{R} l l_{R}}$ & $2 Q_{\text {lvle }}$ \\
$O_{q q_{R} q q_{R}}^{(1)}$ & $2 Q_{q u q d}^{(1)}$ \\
$O_{q q_{R} q q_{R}}^{(8)}$ & $2 Q_{q u q d}^{(8)}$ \\
$O_{l l_{R} q q_{R}}^{(1) \pm}$ & $-Q_{\text {lequ }}^{(1)} \pm Q_{l \nu q d}^{(1)}$ \\
$O_{l l_{R} q q_{R}}^{(3) \pm}$ & $-Q_{\text {lequ }}^{(3)} \pm Q_{l \nu q d}^{(3)}$ \\
\hline \hline
\end{tabular}

TABLE V. A translation dictionary: the custodial basis Wilson coefficients $a_{i}$ in terms of the Warsaw basis Wilson coefficients $C_{i}$.

\begin{tabular}{lc}
\hline \hline $1: X^{3}$ & \\
\hline$a_{G}$ & $C_{G}$ \\
$a_{\tilde{G}}$ & $C_{\tilde{G}}$ \\
$a_{W}$ & $C_{W}$ \\
$a_{\tilde{W}}$ & $C_{\tilde{W}}$ \\
\hline \hline $2: H^{6}$ & \\
\hline$a_{H}$ & $\frac{1}{8} C_{H}$ \\
\hline \hline
\end{tabular}

3: $H^{4} D^{2}$

\begin{tabular}{lc}
\hline$a_{H \square}$ & $C_{H \square}-\frac{1}{4} C_{H D}$ \\
$a_{H D}$ & $\frac{1}{4} C_{H D}$ \\
\hline
\end{tabular}

5: $\bar{\psi} \psi H^{3}+$ H.c.

\begin{tabular}{ll}
\hline$a_{l H}^{ \pm}$ & $\frac{1}{4}\left(C_{\nu H} \pm C_{e H}\right)$ \\
$a_{q H}^{ \pm}$ & $\frac{1}{4}\left(C_{u H} \pm C_{d H}\right)$ \\
\hline \hline
\end{tabular}

\begin{tabular}{lc}
$4: X^{2} H^{2}$ & \\
\hline$a_{H G}$ & $\frac{1}{2} C_{H G}$ \\
$a_{H \tilde{G}}$ & $\frac{1}{2} C_{H \tilde{G}}$ \\
$a_{H W}$ & $\frac{1}{2} C_{H W}$ \\
$a_{H \tilde{W}}$ & $\frac{1}{2} C_{H \tilde{W}}$ \\
$a_{H B}$ & $\frac{1}{2} C_{H B}$ \\
$a_{H \tilde{B}}$ & $\frac{1}{2} C_{H \tilde{B}}$ \\
$a_{H W B}$ & $-\frac{1}{2} C_{H W B}$ \\
$a_{H \tilde{W} B}$ & $-\frac{1}{2} C_{H \tilde{W} B}$ \\
\hline \hline
\end{tabular}

6: $\bar{\psi} \psi X H+$ H.c.

\begin{tabular}{lc}
\hline$a_{l W}^{ \pm}$ & $\frac{1}{2}\left(C_{\nu W} \pm C_{e W}\right)$ \\
$a_{l B}^{ \pm}$ & $\frac{1}{2}\left(C_{\nu B} \pm C_{e B}\right)$ \\
$a_{q G}^{ \pm}$ & $\frac{1}{2}\left(C_{u G} \pm C_{d G}\right)$ \\
$a_{q W}^{ \pm}$ & $\frac{1}{2}\left(C_{u W} \pm C_{d W}\right)$ \\
$a_{q B}^{ \pm}$ & $\frac{1}{2}\left(C_{u B} \pm C_{d B}\right)$ \\
\hline \hline
\end{tabular}

7: $\bar{\psi} \psi H^{2} D$

\begin{tabular}{lc}
\hline$a_{H l}^{(1)}$ & $-C_{H l}^{(1)}$ \\
$a_{H l}^{(3)}$ & $C_{H l}^{(3)}$ \\
$a_{H q}^{(1)}$ & $-C_{H q}^{(1)}$ \\
$a_{H q}^{(3)}$ & $C_{H q}^{(3)}$ \\
$a_{H l_{R}}^{(1) \pm}$ & $-\frac{1}{2}\left(C_{H \nu} \pm C_{H e}\right)+\frac{1}{4}\left( \pm C_{H \nu e}-C_{H \nu e}^{*}\right)$ \\
$a_{H l_{R}}^{(3) \pm}$ & $\frac{1}{4}\left( \pm C_{H \nu e}+C_{H \nu e}^{*}\right)$ \\
$a_{H q_{R}}^{(1) \pm}$ & $-\frac{1}{2}\left(C_{H u} \pm C_{H d}\right)+\frac{1}{4}\left( \pm C_{H u d}-C_{H u d}^{*}\right)$ \\
$a_{H q_{R}}^{(3) \pm}$ & $\frac{1}{4}\left( \pm C_{H u d}+C_{H u d}^{*}\right)$ \\
\hline \hline $8:(\bar{L} L)(\bar{L} L)$ & \\
\hline$a_{l l}$ & \\
\hline
\end{tabular}

(Table continued) 
TABLE V. (Continued)

\begin{tabular}{ll}
\hline \hline $8:(\bar{L} L)(\bar{L} L)$ & \\
\hline$a_{q q}^{(1)}$ & $C_{q q}^{(1)}$ \\
$a_{q q}^{(3)}$ & $C_{q q}^{(3)}$ \\
$a_{l q}^{(1)}$ & $C_{l q}^{(1)}$ \\
$a_{l q}^{(3)}$ & $C_{l q}^{(3)}$ \\
\hline \hline
\end{tabular}

8: $(\bar{R} R)(\bar{R} R)$

\begin{tabular}{lc}
\hline$a_{l_{R} l_{R}}^{ \pm \pm}$ & $\frac{1}{4}\left(C_{\nu \nu}+C_{e e} \pm C_{\nu e}\right)$ \\
$a_{l_{R} l_{R}}^{+-}$ & $\frac{1}{2}\left(C_{\nu \nu}-C_{e e}\right)$ \\
$a_{q_{R} q_{R}}^{(1) \pm \pm}$ & $\frac{1}{4}\left[\left(C_{u u}+C_{d d}\right) \pm C_{u d}^{(1)}-\frac{1}{4} C_{u d}^{(8)} \pm\left(\frac{1}{4}-\frac{1}{2 N_{c}}\right) C_{u d}^{(8)}\right]$ \\
$a_{q_{R} q_{R}}^{(1)+-}$ & $\frac{1}{2}\left(C_{u u}-C_{d d}\right)$ \\
$a_{q_{R} q_{R}}^{(3)++}$ & $\frac{1}{8} C_{u d}^{(8)}$ \\
$a_{l_{R} q_{R}}^{(1)+ \pm}$ & $\frac{1}{4}\left[\left(C_{\nu u}+C_{e u}\right) \pm\left(C_{\nu d}+C_{e d}\right)\right]$ \\
$a_{l_{R} q_{R}}^{(1)- \pm}$ & $\frac{1}{4}\left[\left(C_{\nu u}-C_{e u}\right) \pm\left(C_{\nu d}-C_{e d}\right)+\left(-C_{\nu e d u} \pm C_{\nu e d u}^{*}\right)\right]$ \\
$a_{l_{R} q_{R}}^{(3)+ \pm}$ & $\frac{1}{4}\left(C_{\nu e d u} \pm C_{\nu e d u}^{*}\right)$ \\
\hline \hline
\end{tabular}

8: $(\bar{L} L)(\bar{R} R)$

\begin{tabular}{ll}
\hline$a_{l_{R}}^{ \pm}$ & $\frac{1}{2}\left(C_{l \nu} \pm C_{l e}\right)$ \\
$a_{l q_{R}}^{ \pm}$ & $\frac{1}{2}\left(C_{l u} \pm C_{l d}\right)$ \\
$a_{q l_{R}}^{ \pm}$ & $\frac{1}{2}\left(C_{q \nu} \pm C_{q e}\right)$ \\
$a_{q q_{R}}^{(1) \pm}$ & $\frac{1}{2}\left[C_{q u}^{(1)} \pm C_{q d}^{(1)}\right]$ \\
$a_{q q_{R}}^{(8) \pm}$ & $\frac{1}{2}\left[C_{q u}^{(8)} \pm C_{q d}^{(8)}\right]$ \\
\hline
\end{tabular}

8: $(\bar{L} R)(\bar{R} L)+$ H.c.

$\overline{a_{l_{R} q_{R} q}^{ \pm}} \quad \frac{1}{2}\left(C_{\text {lvuq }} \pm C_{\text {ledq }}\right)$

8: $(\bar{L} R)(\bar{L} R)+$ H.c.

\begin{tabular}{lc}
\hline$a_{l l_{R} l_{R}}$ & $\frac{1}{2} C_{\text {lvle }}$ \\
$a_{q q_{R} q q_{R}}^{(1)}$ & $\frac{1}{2} C_{q u q d}^{(1)}$ \\
$a_{q q_{R} q q_{R}}^{(1)}$ & $\frac{1}{2} C_{q u q d}^{(8)}$ \\
$a_{l l_{R} \pm q_{R}}^{(1) \pm}$ & $\frac{1}{2}\left[-C_{\text {lequ }}^{(1)} \pm C_{\text {lvqd }}^{(1)}\right]$ \\
$a_{\left.l_{l_{R} q q_{R}}\right)}^{(3)}$ & $\frac{1}{2}\left[-C_{\text {lequ }}^{(3)} \pm C_{\text {lvqd }}^{(3)}\right]$ \\
\hline \hline
\end{tabular}

TABLE VI. A translation dictionary: the Warsaw basis Wilson coefficients $C_{i}$ in terms of the custodial basis Wilson coefficients $a_{i}$.

\begin{tabular}{lc}
\hline \hline $1: X^{3}$ & \\
\hline$C_{G}, C_{\tilde{G}}$ & $a_{G}, a_{\tilde{G}}$ \\
$C_{W}, C_{\tilde{W}}$ & $a_{W}, a_{\tilde{W}}$ \\
\hline \hline $2: H^{6}$ & \\
\hline$C_{H}$ & $8 a_{H}$ \\
\hline \hline
\end{tabular}

(Table continued)
TABLE VI. (Continued)

\begin{tabular}{lc}
\hline $3: H^{4} D^{2}$ & \\
\hline$C_{H \square}$ & $\begin{array}{c}a_{H \square}+a_{H D} \\
4 a_{H D}\end{array}$ \\
$C_{H D}$ & \\
\hline \hline $5: \bar{\psi} \psi H^{3}+$ H.c. & \\
\hline$C_{\nu H}, C_{e H}$ & $2\left(a_{l H}^{+} \pm a_{l H}^{-}\right)$ \\
$C_{u H}, C_{d H}$ & $2\left(a_{q H}^{+} \pm a_{q H}^{-}\right)$ \\
\hline \hline $4: X^{2} H^{2}$ & \\
\hline$C_{H G}$ & $2 a_{H G}$ \\
$C_{H \tilde{G}}$ & $2 a_{H \tilde{G}}$ \\
$C_{H W}$ & $2 a_{H W}$ \\
$C_{H \tilde{W}}$ & $2 a_{H \tilde{W}}$ \\
$C_{H B}$ & $2 a_{H B}$ \\
$C_{H \tilde{B}}$ & $2 a_{H \tilde{B}}$ \\
$C_{H W B}$ & $-2 a_{H W B}$ \\
$C_{H \tilde{W} B}$ & $-2 a_{H \tilde{W} B}$ \\
\hline \hline $6: \tilde{y}$ & \\
\hline \hline
\end{tabular}

6: $\bar{\psi} \psi X H+$ H.c.

\begin{tabular}{|c|c|}
\hline $\begin{array}{l}C_{\nu W}, C_{e W} \\
C_{\nu B}, C_{e B} \\
C_{u G}, C_{d G} \\
C_{u W}, C_{d W} \\
C_{u B}, C_{d B}\end{array}$ & $\begin{array}{c}a_{l W}^{+} \pm a_{l W}^{-} \\
a_{l B}^{+} \pm a_{l B}^{-} \\
a_{q G}^{+} \pm a_{q G}^{-} \\
a_{q W}^{+} \pm a_{q W}^{-} \\
a_{q B}^{+}+a_{q B}^{-}\end{array}$ \\
\hline \multicolumn{2}{|l|}{$7: \bar{\psi} \psi H^{2} D$} \\
\hline $\begin{array}{l}C_{H l}^{(1)} \\
C_{H l}^{(3)} \\
C_{H q}^{(1)} \\
C_{H q}^{(3)} \\
C_{H \nu}, C_{H e} \\
C_{H \nu e} \\
C_{H u}, C_{H d} \\
C_{H u d}\end{array}$ & $\begin{array}{c}-a_{H l}^{(1)} \\
a_{H l}^{(3)} \\
-a_{H q}^{(1)} \\
a_{H q}^{(3)} \\
-a_{H l_{R}}^{(1)+} \mp a_{H l_{R}}^{(1)-} \mp a_{H l_{R}}^{(3)+}-a_{H l_{R}}^{(3)-} \\
2\left[a_{H l_{R}}^{(3)+}-a_{H l_{R}}^{(3)-}\right] \\
-a_{H q_{R}}^{(1)+} \mp a_{H q_{R}}^{(1)-} \mp a_{H q_{R}}^{(3)+}-a_{H q_{R}}^{(3)-} \\
2\left[a_{H q_{R}}^{(3)+}-a_{H q_{R}}^{(3)-}\right]\end{array}$ \\
\hline
\end{tabular}

8: $(\bar{L} L)(\bar{L} L)$

\begin{tabular}{lc}
\hline$C_{l l}$ & $a_{l l}$ \\
$C_{q q}^{(1)}$ & $a_{q q}^{(1)}$ \\
$C_{q q}^{(3)}$ & $a_{q q}^{(3)}$ \\
$C_{l q}^{(1)}$ & $a_{l q}^{(1)}$ \\
$C_{l q}^{(3)}$ & $a_{l q}^{(3)}$ \\
\hline \hline
\end{tabular}

\begin{tabular}{ll}
$8:(\bar{R} R)(\bar{R} R)$ & \\
\hline$C_{\nu \nu}$ & $a_{l_{R} l_{R}}^{++}+a_{l_{R} l_{R}}^{-}+a_{l_{R} l_{R}}^{+-}$ \\
$C_{e e}$ & $a_{l_{R} l_{R}}^{++}+a_{l_{R} l_{R}}^{-}-a_{l_{R} l_{R}}^{+-}$
\end{tabular}

(Table continued) 
TABLE VI. (Continued)

\begin{tabular}{|c|c|}
\hline \multicolumn{2}{|c|}{ 8: $(\bar{R} R)(\bar{R} R)$} \\
\hline$C_{\nu e}$ & $2\left(a_{l_{p} l_{p}}^{++}-a_{l_{p} l_{p}}^{--}\right)$ \\
\hline$C_{u u}$ & $a_{q_{R} q_{R}}^{(1)++}+a_{q_{R} q_{R}}^{(1)--}+a_{q_{R} q_{R}}^{(1)+-}+a_{q_{R} q_{R}}^{(3)++}$ \\
\hline$C_{d d}$ & $a_{q_{R} q_{R}}^{(1)++}+a_{q_{R} q_{R}}^{(1)--}-a_{q_{R} q_{R}}^{(1)+-}+a_{q_{R} q_{R}}^{(3)++}$ \\
\hline & $2\left[a_{q_{R} q_{R}}^{(1)++}-a_{q_{R} q_{R}}^{(1)--}\right]+\left(\frac{4}{N_{c}}-2\right) a_{q_{R} q_{R}}^{(3)++}$ \\
\hline$C_{u d}^{(8)}$ & $8 a_{q_{R} q_{R}}^{(3)++}$ \\
\hline$C_{\nu u}$ & $a_{l_{R} q_{R}}^{(1)++}+a_{l_{R} q_{R}}^{(1)--}+a_{l_{R} q_{R}}^{(1)+-}+a_{l_{R} q_{R}}^{(1)-+}+a_{l_{R} q_{R}}^{(3)++}+a_{l_{R} q_{R}}^{(3)+-}$ \\
\hline$C_{\nu d}$ & $a_{l_{R} q_{R}}^{(1)++}-a_{l_{R} q_{R}}^{(1)--}-a_{l_{R} q_{R}}^{(1)+-}+a_{l_{R} q_{R}}^{(1)-+}-a_{l_{R} q_{R}}^{(3)++}+a_{l_{R} q_{R}}^{(3)+-}$ \\
\hline$C_{e u}$ & $a_{l_{R} q_{R}}^{(1)++}-a_{l_{R} q_{R}}^{(1)--}+a_{l_{R} q_{R}}^{(1)+-}-a_{l_{R} q_{R}}^{(1)-+}-a_{l_{R} q_{R}}^{(3)++}-a_{l_{R} q_{R}}^{(3)+-}$ \\
\hline$C_{e d}$ & $a_{l_{R} q_{R}}^{(1)++}+a_{l_{R} q_{R}}^{(1)--}-a_{l_{R} q_{R}}^{(1)+-}-a_{l_{R} q_{R}}^{(1)-+}+a_{l_{R} q_{R}}^{(3)++}-a_{l_{R} q_{R}}^{(3)+-}$ \\
\hline$C_{\text {vedu }}$ & $2\left[a_{l_{R} q_{R}}^{(3)^{2}++}+a_{l_{R} q_{R}}^{(3)+-}\right]$ \\
\hline
\end{tabular}

8: $(\bar{L} L)(\bar{R} R)$

\begin{tabular}{lc}
\hline$C_{l \nu}$ & $a_{l l_{R}}^{+}+a_{l l_{R}}^{-}$ \\
$C_{l e}$ & $a_{l l_{R}}^{+}-a_{l l_{R}}^{-}$ \\
$C_{l u}$ & $a_{l q_{R}}^{+}+a_{l q_{R}}^{-}$ \\
$C_{l d}$ & $a_{l q_{R}}^{+}-a_{l q_{R}}^{-}$ \\
$C_{q \nu}$ & $a_{q l_{R}}^{+}+a_{q l_{R}}^{-}$ \\
$C_{q e}$ & $a_{q l_{R}}^{+}-a_{q l_{R}}^{-}$ \\
$C_{q u}^{(1)}$ & $a_{q q_{R}}^{(1)+}+a_{q q_{R}}^{(1)-}$ \\
$C_{q d}^{(1)}$ & $a_{q q_{R}}^{(1)+}-a_{q q_{R}}^{(1)-}$ \\
$C_{q u}^{(8)}$ & $a_{q q_{R}}^{(8)+}+a_{q q_{R}}^{(8)-}$ \\
$C_{q d}^{(8)}$ & $a_{q q_{R}}^{(8)+}-a_{q q_{R}}^{(8)-}$ \\
\hline \hline
\end{tabular}

8: $(\bar{L} R)(\bar{R} L)+$ H.c.

\begin{tabular}{|c|c|}
\hline$C_{l v u q}, C_{l e d q}$ & $a_{l l_{R} q_{R} q}^{+} \pm a_{l l_{R} q_{R} q}^{-}$ \\
\hline
\end{tabular}

8: $(\bar{L} R)(\bar{L} R)+$ H.c.

$C_{\text {lule }}$

$C_{\text {quqd }}^{(1)}, C_{\text {quqd }}^{(8)}$

$C_{\text {lvqd }}^{(1)}, C_{\text {lequ }}^{(1)}$

$C_{\text {lvqd }}^{(3)}, C_{\text {lequ }}^{(3)}$

$2 a_{l l_{R} l l_{R}}$
$2 a_{q q_{R}}^{(1)} q q_{R}, 2 a_{q q_{R} q q_{R}}^{(8)}$
$\mp a_{l l_{R} q q_{R}}^{(1)}-a_{l l_{R} q q_{R}}^{(1)-}$
$\mp a_{l l_{R} q q_{R}}^{(3)+}-a_{l l_{R} q q_{R}}^{(3)-}$

TABLE VII. Reducing 2 SMEFT to SMEFT: the left (right) column shows the constraints on the Wilson coefficients in the Warsaw (custodial) basis.

\begin{tabular}{lc}
\hline \hline $\begin{array}{l}\nu \text { SMEF } \rightarrow \text { SMEFT } \\
\text { in the Warsaw basis }\end{array}$ & $\begin{array}{c}\nu \text { SMEFT } \rightarrow \text { SMEFT } \\
\text { in the custodial basis }\end{array}$ \\
\hline$C_{\nu H}=0$ & $a_{l H}^{+}=-a_{l H}^{-}$ \\
$C_{\nu W}=C_{\nu B}=0$ & $a_{l W}^{+}=-a_{l W}^{-}, a_{l B}^{+}=-a_{l B}^{-}$ \\
$C_{H \nu e}=C_{H \nu e}^{*}=0$ & $a_{H l_{R}}^{(3)+}=a_{H l_{R}}^{(3)-}=0$ \\
$C_{H \nu}=0$ & $a_{H l_{R}}^{(1)+}=-a_{H l_{R}}^{(1)-}$ \\
$C_{\nu \nu}=C_{\nu e}=0$ & $a_{l_{R} l_{R}}^{++}=a_{l_{R} l_{R}}^{-}=-\frac{1}{2} a_{l_{R} l_{R}}^{+-}$ \\
$C_{\nu e d u}=C_{\nu e d u}^{*}=0$ & $a_{l_{R} q_{R}}^{(3)++}=a_{l_{R} q_{R}}^{(3)+-}=0$ \\
$C_{\nu u}=C_{\nu d}=0$ & $a_{l_{R} q_{R}}^{(1)++}=-a_{l_{R} q_{R}}^{(1)-+}, a_{l_{R} q_{R}}^{(1)+-}=-a_{l_{R} q_{R}}^{(1)--}$ \\
$C_{l \nu}=C_{q \nu}=0$ & $a_{l l_{R}}^{+}=-a_{l l_{R}}^{-}, a_{q l_{R}}^{+}=-a_{q l_{R}}^{-}$ \\
$C_{l \nu u q}=0$ & $a_{l l_{R} q_{R} q}^{+}=-a_{l l_{R} q_{R} q}$ \\
$C_{l \nu l e}=0$ & $a_{l l_{R} l l_{R}}=0$ \\
$C_{l \nu q d}^{(1)}=C_{l \nu q d}^{(3)}=0$ & $a_{l l_{R} q q_{R}}^{(1)+}=-a_{l l_{R} q q_{R}}^{(1)-}, a_{l l_{R} q q_{R}}^{(3)+}=-a_{l l_{R} q q_{R}}^{(3)-}$ \\
\hline \hline
\end{tabular}

dim-6 2 SMEFT (suppressing flavor indices). These operators are recombined to form our custodial basis summarized in Table III. Table IV provides an explicit translation dictionary between the operators in these two operator bases. Translation dictionaries between the Wilson coefficients $C_{i}$ and $a_{i}$, in both directions, are further provided in Tables V and VI. Table VII summarizes the restrictions on the Wilson coefficients $C_{i}$ and $a_{i}$ to reduce $\nu$ SMEFT back to SMEFT.

Our notations in Table III are also a bit compact. For example, we sometimes use $O^{ \pm}$to group custodial-preserving/-violating operators together which, respectively, involves $P_{ \pm}$. Such examples include $O_{l H}^{ \pm}, O_{q H}^{ \pm}, O_{l W}^{ \pm}, O_{q G}^{ \pm}$, $O_{q W}^{ \pm}, O_{H l_{R}}^{(3) \pm}$, and $O_{H q_{R}}^{(3) \pm}$. A similar kind of notation is also applied to some custodial-preserving four-fermion operators that break the isospin $S U(2)_{R q_{R}}$ or $S U(2)_{R l_{R}}$. In particular, the notation $\mathrm{O}^{--}$implies that the operator violates both the lepton and quark isospin.
[1] The ALEPH, DELPHI, L3, OPAL, SLD Collaborations, the LEP Electroweak Working Group, the SLD Electroweak and Heavy Flavour Groups, Precision electroweak measurements on the $\mathrm{Z}$ resonance, Phys. Rep. 427, 257 (2006).
[2] The ALEPH, DELPHI, L3, OPAL Collaborations, the LEP Electroweak Working Group, Electroweak measurements in electron-positron collisions at W-boson-pair energies at LEP, Phys. Rep. 532, 119 (2013). 
[3] M.E. Peskin and T. Takeuchi, Estimation of oblique electroweak corrections, Phys. Rev. D 46, 381 (1992).

[4] P. Sikivie, L. Susskind, M. B. Voloshin, and V. I. Zakharov, Isospin breaking in technicolor models, Nucl. Phys. B173, 189 (1980).

[5] C. T. Hill and E. H. Simmons, Strong dynamics and electroweak symmetry breaking, Phys. Rep. 381, 235 (2003); Erratum, Phys. Rep. 390, 553 (2004).

[6] H. Georgi and D. B. Kaplan, Composite Higgs and custodial SU(2), Phys. Lett. 145B, 216 (1984).

[7] M. J. Dugan, H. Georgi, and D. B. Kaplan, Anatomy of a composite Higgs model, Nucl. Phys. B254, 299 (1985).

[8] K. Agashe, R. Contino, and A. Pomarol, The minimal composite Higgs model, Nucl. Phys. B719, 165 (2005).

[9] K. Agashe, R. Contino, L. Da Rold, and A. Pomarol, A custodial symmetry for $Z b \bar{b}$, Phys. Lett. B 641, 62 (2006).

[10] G. F. Giudice, C. Grojean, A. Pomarol, and R. Rattazzi, The strongly-interacting light Higgs, J. High Energy Phys. 06 (2007) 045.

[11] C. Csaki, J. Hubisz, G. D. Kribs, P. Meade, and J. Terning, Big corrections from a little Higgs, Phys. Rev. D 67, 115002 (2003).

[12] J. L. Hewett, F. J. Petriello, and T. G. Rizzo, Constraining the littlest Higgs, J. High Energy Phys. 10 (2003) 062.

[13] S. Chang, A 'Littlest Higgs' model with custodial SU(2) symmetry, J. High Energy Phys. 12 (2003) 057.

[14] H.-C. Cheng and I. Low, TeV symmetry and the little hierarchy problem, J. High Energy Phys. 09 (2003) 051.

[15] M.-C. Chen and S. Dawson, One loop radiative corrections to the rho parameter in the littlest Higgs model, Phys. Rev. D 70, 015003 (2004).

[16] A. Delgado, M. Garcia-Pepin, B. Ostdiek, and M. Quiros, Dark matter from the supersymmetric custodial triplet model, Phys. Rev. D 92, 015011 (2015).

[17] G. D. Kribs, A. Martin, and T. Tong, Effective theories of dark mesons with custodial symmetry, J. High Energy Phys. 08 (2019) 020.

[18] S.-M. Choi, H. M. Lee, Y. Mambrini, and M. Pierre, Vector SIMP dark matter with approximate custodial symmetry, J. High Energy Phys. 07 (2019) 049.

[19] M. J. G. Veltman, Limit on mass differences in the Weinberg model, Nucl. Phys. B123, 89 (1977).

[20] P. Zyla et al. (Particle Data Group Collaboration), Review of Particle Physics, Prog. Theor. Exp. Phys. 2020, 083C01 (2020).

[21] I. Maksymyk, C. Burgess, and D. London, Beyond S, T and U, Phys. Rev. D 50, 529 (1994).

[22] C. Burgess, S. Godfrey, H. Konig, D. London, and I. Maksymyk, A global fit to extended oblique parameters, Phys. Lett. B 326, 276 (1994).

[23] A. Kundu and P. Roy, A general treatment of oblique parameters, Int. J. Mod. Phys. A 12, 1511 (1997).

[24] R. Barbieri, A. Pomarol, R. Rattazzi, and A. Strumia, Electroweak symmetry breaking after LEP-1 and LEP-2, Nucl. Phys. B703, 127 (2004).

[25] G. Aad et al. (ATLAS Collaboration), Observation of a new particle in the search for the Standard Model Higgs boson with the ATLAS detector at the LHC, Phys. Lett. B 716, 1 (2012).
[26] S. Chatrchyan et al. (CMS Collaboration), Observation of a new boson at a mass of $125 \mathrm{GeV}$ with the CMS experiment at the LHC, Phys. Lett. B 716, 30 (2012).

[27] S. Weinberg, Baryon and Lepton Nonconserving Processes, Phys. Rev. Lett. 43, 1566 (1979).

[28] S. Weinberg, Varieties of baryon and lepton nonconservation, Phys. Rev. D 22, 1694 (1980).

[29] W. Buchmuller and D. Wyler, Effective Lagrangian analysis of new interactions and flavor conservation, Nucl. Phys. B268, 621 (1986).

[30] B. Grzadkowski, M. Iskrzynski, M. Misiak, and J. Rosiek, Dimension-six terms in the standard model Lagrangian, J. High Energy Phys. 10 (2010) 085.

[31] J. Elias-Miró, C. Grojean, R. S. Gupta, and D. Marzocca, Scaling and tuning of EW and Higgs observables, J. High Energy Phys. 05 (2014) 019.

[32] E. E. Jenkins and A. V. Manohar, Algebraic structure of lepton and quark flavor invariants and $C P$ violation, J. High Energy Phys. 10 (2009) 094.

[33] A. Hanany, E. E. Jenkins, A. V. Manohar, and G. Torri, Hilbert series for flavor invariants of the standard model, J. High Energy Phys. 03 (2011) 096.

[34] L. Lehman and A. Martin, Hilbert Series for Constructing Lagrangians: expanding the phenomenologist's toolbox, Phys. Rev. D 91, 105014 (2015).

[35] B. Henning, X. Lu, T. Melia, and H. Murayama, Hilbert series and operator bases with derivatives in effective field theories, Commun. Math. Phys. 347, 363 (2016).

[36] B. Henning, X. Lu, T. Melia, and H. Murayama, Operator bases, $S$-matrices, and their partition functions, J. High Energy Phys. 10 (2017) 199.

[37] L. Lehman, Extending the Standard Model effective field theory with the complete set of dimension-7 operators, Phys. Rev. D 90, 125023 (2014).

[38] L. Lehman and A. Martin, Low-derivative operators of the Standard Model effective field theory via Hilbert series methods, J. High Energy Phys. 02 (2016) 081.

[39] B. Henning, X. Lu, T. Melia, and H. Murayama, 2, 84, 30, 993, 560, 15456, 11962, 261485, ...: Higher dimension operators in the SM EFT, J. High Energy Phys. 08 (2017) 016; Erratum, J. High Energy Phys. 09 (2019) 19.

[40] C. B. Marinissen, R. Rahn, and W. J. Waalewijn, ..., 83106786, 114382724, 1509048322, 2343463290, $27410087742, \ldots$ Efficient hilbert series for effective theories, Phys. Lett. B 808, 135632 (2020).

[41] R. Alonso, E. E. Jenkins, A. V. Manohar, and M. Trott, Renormalization group evolution of the standard model dimension six operators III: Gauge coupling dependence and phenomenology, J. High Energy Phys. 04 (2014) 159.

[42] J. D. Wells and Z. Zhang, Effective theories of universal theories, J. High Energy Phys. 01 (2016) 123.

[43] J. D. Wells and Z. Zhang, Renormalization group evolution of the universal theories EFT, J. High Energy Phys. 06 (2016) 122.

[44] M. Trott, On the consistent use of Constructed Observables, J. High Energy Phys. 02 (2015) 046.

[45] M. Trott, EWPD in the SMEFT and the $\mathcal{O}\left(y_{t}^{2}, \lambda\right)$ one loop $Z$ decay width, in 52nd Rencontres de Moriond on EW Interactions and Unified Theories (2017), pp. 63-70. 
[46] I. Brivio and M. Trott, Scheming in the SMEFT... and a reparameterization invariance!, J. High Energy Phys. 07 (2017) 148; 05 (2018) 136(A).

[47] C. Arzt, M. B. Einhorn, and J. Wudka, Patterns of deviation from the standard model, Nucl. Phys. B433, 41 (1995).

[48] M. B. Einhorn and J. Wudka, The bases of effective field theories, Nucl. Phys. B876, 556 (2013).

[49] N. Craig, M. Jiang, Y.-Y. Li, and D. Sutherland, Loops and trees in generic EFTs, J. High Energy Phys. 08 (2020) 086.

[50] E. E. Jenkins, A. V. Manohar, and M. Trott, Renormalization group evolution of the standard model dimension six operators I: Formalism and lambda dependence, J. High Energy Phys. 10 (2013) 087.

[51] E. E. Jenkins, A. V. Manohar, and M. Trott, Renormalization group evolution of the standard model dimension six operators II: Yukawa dependence, J. High Energy Phys. 01 (2014) 035.

[52] Y. Liao and X.-D. Ma, Operators up to dimension seven in standard model effective field theory extended with sterile neutrinos, Phys. Rev. D 96, 015012 (2017).

[53] J. Elias-Miro, J. R. Espinosa, E. Masso, and A. Pomarol, Higgs windows to new physics through $d=6$ operators: Constraints and one-loop anomalous dimensions, J. High Energy Phys. 11 (2013) 066.

[54] I. Brivio and M. Trott, The standard model as an effective field theory, Phys. Rep. 793, 1 (2019).

[55] J. Baglio, S. Dawson, and I. M. Lewis, NLO effects in EFT fits to $W^{+} W^{-}$production at the LHC, Phys. Rev. D 99, 035029 (2019).

[56] W. Skiba, Effective field theory and precision electroweak measurements, in TASI 2009 (2011), pp. 5-70.

[57] Z. U. Khandker, D. Li, and W. Skiba, Electroweak corrections from triplet scalars, Phys. Rev. D 86, 015006 (2012).
[58] J. de Blas, M. Chala, M. Perez-Victoria, and J. Santiago, Observable effects of general new scalar particles, J. High Energy Phys. 04 (2015) 078.

[59] C.-W. Chiang and R. Huo, Standard model effective field theory: Integrating out a generic scalar, J. High Energy Phys. 09 (2015) 152.

[60] S. A. R. Ellis, J. Quevillon, T. You, and Z. Zhang, Mixed heavy-light matching in the Universal One-Loop Effective Action, Phys. Lett. B 762, 166 (2016).

[61] S. Dawson and C.W. Murphy, Standard Model EFT and extended scalar sectors, Phys. Rev. D 96, 015041 (2017).

[62] J. Heeck, Unbroken B-L symmetry, Phys. Lett. B 739, 256 (2014).

[63] G. D’Ambrosio, G. F. Giudice, G. Isidori, and A. Strumia, Minimal flavor violation: An effective field theory approach, Nucl. Phys. B645, 155 (2002).

[64] F. del Aguila, M. Perez-Victoria, and J. Santiago, Observable contributions of new exotic quarks to quark mixing, J. High Energy Phys. 09 (2000) 011.

[65] F. del Aguila, J. de Blas, and M. Perez-Victoria, Effects of new leptons in electroweak precision data, Phys. Rev. D 78, 013010 (2008).

[66] A. Crivellin, F. Kirk, C. A. Manzari, and M. Montull, Global electroweak fit and vector-like leptons in light of the cabibbo angle anomaly, J. High Energy Phys. 12 (2020) 166.

[67] M. Jiang, N. Craig, Y.-Y. Li, and D. Sutherland, Complete one-loop matching for a singlet scalar in the standard model EFT, J. High Energy Phys. 02 (2019) 031.

[68] U. Haisch, M. Ruhdorfer, E. Salvioni, E. Venturini, and A. Weiler, Singlet night in Feynman-ville: one-loop matching of a real scalar, J. High Energy Phys. 04 (2020) 164. 